\title{
THE SOCIAL CONSTRUCTION OF KNOWLEDGE IN THE FIELD OF SPORT MANAGEMENT: \\ A SOCIAL NETWORK PERSPECTIVE
}

\author{
DISSERTATION \\ Presented in Partial Fulfillment of the Requirements for \\ the Degree Doctor of Philosophy in the Graduate \\ School of The Ohio State University
}

By

Catherine Quatman, B.S., M.A.

$* * * * *$

The Ohio State University

2006

Dissertation Committee:

Approved by

Dr. Packianathan Chelladurai, Co-Advisor

Dr. Janet Fink

Dr. James Moody, Co-Advisor

Advisor

Dr. Brian Turner

College of Education 



\begin{abstract}
As the North American Society for Sport Management (NASSM) is fast approaching the $25^{\text {th }}$ anniversary of its inception, the intent of this study was to critically review and empirically explore the social scientific structure of the field of sport management in North America. Integrating a network perspective, the ideas presented offer a unique and compelling approach toward identifying the underlying social structure and potential socio-structural barriers to the diffusion of innovation throughout the network of scholars.
\end{abstract}

A network model of coauthorship patterns was generated using several rounds of sampling and archival data collection. The observed network structure was explored both quantitatively and qualitatively for meaningful patterns. The results of the study were intended to essentially tell a story of the evolution and current state of the field of sport management's coauthorship structure and identify potential socio-structural barriers present in the network. Among the themes that emerged included the structural dominance of one particular institution and the presence of a structural gap between researchers in the United States and Canada. As this study was exploratory in nature, the themes developed were not meant to be conclusive but rather lay the foundation for future research directions.

The findings of the study were tied to theoretical, methodological, and practical implications for the diffusion of ideas and practices throughout the network structure. In 
addition, the secondary thrust of this study was to fundamentally introduce social network analysis as an alternative methodological approach to scholars in the field of sport management. By incorporating some of the techniques of social network analysis into the process of a critical investigation of the social construction of knowledge in the field, it is the authors' hope to insightfully delineate how network analytic techniques can provide an opportunity to explore more diverse and robust research questions in the future. 
Dedicated to my parents and sisters.

Thank you for all of your love and support. 


\section{ACKNOWLEDGMENTS}

There were so many people who assisted and supported me during my doctoral studies that deserve recognition far beyond the credits I can give in an acknowledgements section.

First and foremost, I am extremely thankful for the insight and willingness of Dr. Packianathan Chelladurai in helping me take on a project entirely outside of his experience and training. His wisdom, enthusiasm, and support throughout my tenure as a graduate student have been inspirational and enlightening. It has been both a pleasure and a blessing to work with such a prestigious and beloved scholar in our field.

I am also very grateful for the support and insight provided by Dr. Janet Fink whose encouragement and wit helped me stay motivated, optimistic, and laughing throughout my tenure at OSU. Dr. Fink often challenged me to find new ways of thinking and articulating my thoughts which I believe will prove to be invaluable lessons for me as an aspiring teacher and researcher in the field.

Likewise, I am thankful to Dr. Brian Turner whose help, encouragement, and exceptional technological skills proved to be a saving grace on more than one occasion during my doctoral career. However, one of the things I am most appreciative for was Dr. Turner's ability to help me find a sense of confidence in my work-something I am not sure I would have found without him. 
I am also extremely grateful for the help of Dr. James Moody who introduced me to the fascinating world of social network analysis. Without his patience, brilliance and aid, it would not have been possible for me to delve into such a daunting field of study.

In addition, the data for my study would never have come to fruition without the help of a number of people. In addition to those I have named above, I would like to thank all those scholars in the field who were willing to participate in the study and reveal some very personal information.

I would also like to thank-

John Cannizzaro--who contributed many hours helping ensure the accuracy of my data.

My mentors at the Department of Recreational Sports and the Sport, Fitness, and Health Program at OSU—Ken Hill, J. Michael Dunn, Chuck Shiebler, and Jae Westfall who were always supportive of my studies, allowing me the flexibility to continue my academic career as well as serving as my support structure along the way.

My doctoral cohort--Annemarie Farrell and Jim Strode who kept me laughing, encouraged, and motivated from the first day I met them. I am so grateful to have had the companionship of two of the most intelligent, witty and caring people I have ever met through some very challenging times over the past three years. In particular, I would like to thank Jim for cooking me omelets and tolerating my whiny cat and Annemarie for making my brain swirl from enlightening philosophical debates for many hours on end.

My good friend and library companion--Heidi Parker who spent many long hours with me at Sullivant Hall during my race for the finish line keeping me fed, sane and in good company. 
Eric Yates—who has been extremely patient, caring and encouraging throughout the entire process. I am so grateful for his love and support and do not know how I would have survived without him.

Finally, I would like to acknowledge my parents Jim and Terri Quatman and my sisters Carmen and Lauren for their unconditional love and endless support. I am continually amazed and forever grateful for their ability to help me strive for my lofty goals, overcome obstacles, and foster my growth as a person. I am utterly humbled by the very active roles they play in my life. 
VITA

October 22, 1980.........................Born - Ashland, Ohio

December 2002................................ Health and Physical Education Edinboro University of Pennsylvania

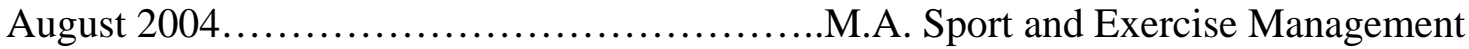
The Ohio State University

2003-2005................................Graduate Administrative Associate The Ohio State University

Department of Recreational Sports

2003-2005...............................Graduate Teaching Associate The Ohio State University School of Physical Activity and Educational Services

\section{FIELDS OF STUDY}

Major Field: $\quad$ College of Education

Physical Activities and Educational Services

Studies in Sport Management

Specialization: $\quad$ Sociology and Social Network Analysis

Minor in Research Methods in Human Resource Development 
TABLE OF CONTENTS

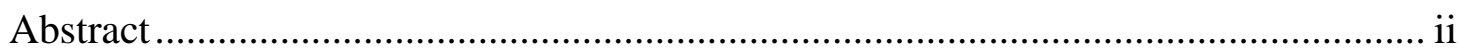

Acknowledgments.............................................................................................. v

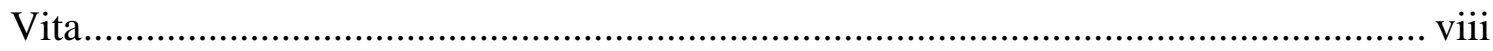

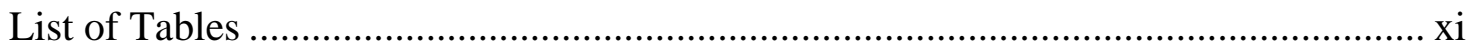

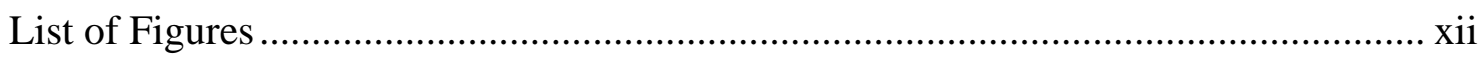

Glossary of Social Network Terms......................................................................... xiii

Chapter

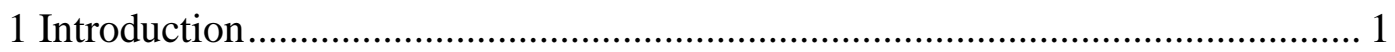

Research on Social Structures and Scientific Knowledge ................................. 5

A Social Network Perspective .......................................................................... 6

Social Network Analysis and the Field of Sport Management........................... 11

Statement of the Problem................................................................................. 12

Purpose of the Study …………………………………................................. 17

Theoretical Premises and Research Questions ................................................... 18

Structural Considerations.......................................................................... 22

Thematic Considerations ................................................................................ 26

Alignment of Informal and Formal Representations of Structure ...................... 30

Limitations and Delimitations............................................................................ 30

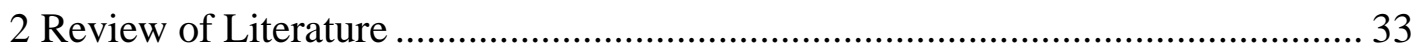

The Status of Sport Management as an Academic Field of Study …………...... 33

The Social Construction of Scientific Knowledge.............................................. 38

The Process of Discovery ………………………………............................... 40

Overview of Metaphysics ................................................................................ 41

Philosophies of Scientific Inquiry.................................................................... 51

Perspectives on the Construction of Knowledge .................................................. 54

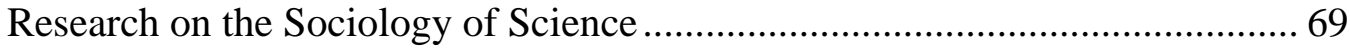

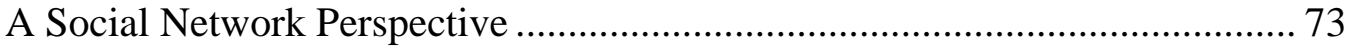

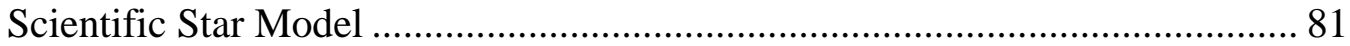

Disciplinary Small World Model......................................................................... 82

Disciplinary Structural Cohesion Model ............................................................ 84

The Social Construction of Knowledge in the Field of Sport Management...... 85 


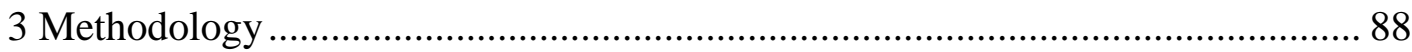

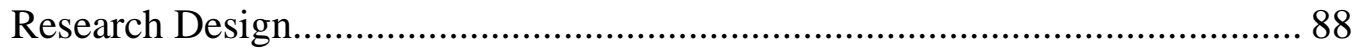

Sampling Procedure and Data Collection ......................................................... 90

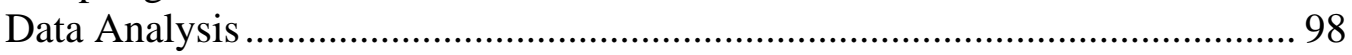

General Collaboration Trends...................................................................... 99

Visualization and Network Evolution............................................................... 99

Quantification and Property Exploration ........................................................... 99

Thematic Development ....................................................................................... 100

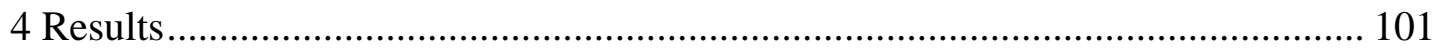

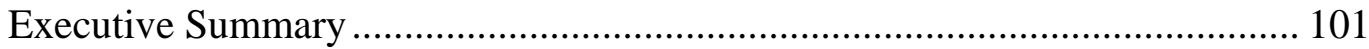

Sampling Procedures ................................................................................... 102

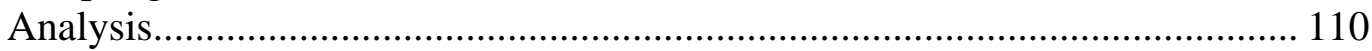

Exploration of General Collaboration Trends................................................. 111

Exploration of the Coauthorship Network Structure ………………………….... 115

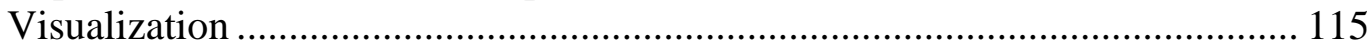

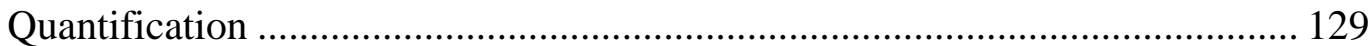

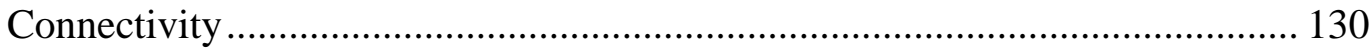

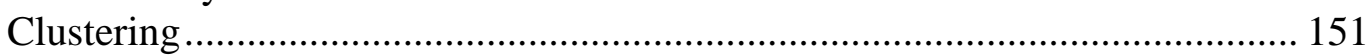

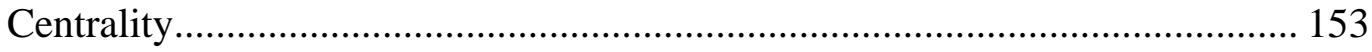

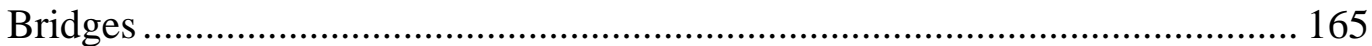

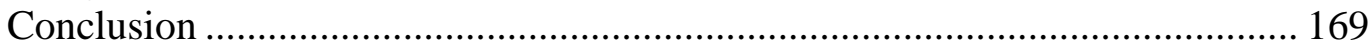

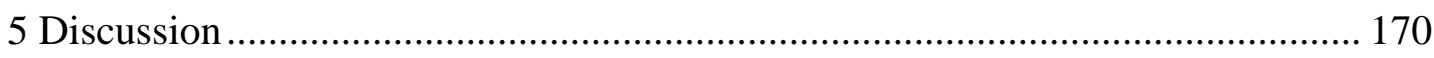

General Collaboration Trends and Evolution of the Field.................................. 171

Socio-Structural Barriers for the Diffusion of Innovative Ideas?........................ 178

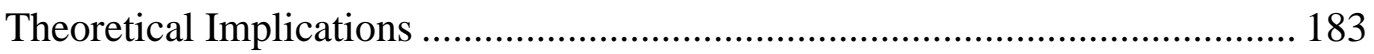

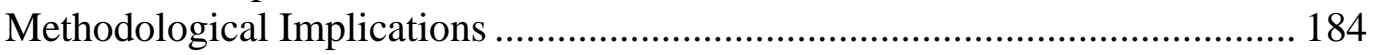

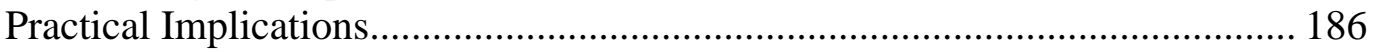

Limitations and Delimitations........................................................................ 187

Future Research Directions.............................................................................. 193

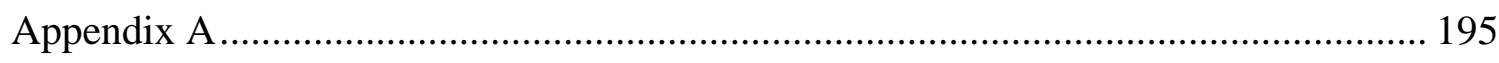

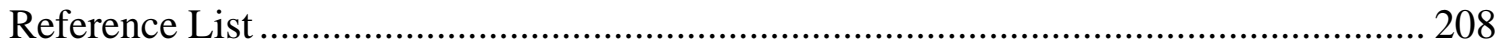




\section{LIST OF TABLES}

Table

4.1 Distributions of Authors per Publication for Each Time Block .................. 112

4.2 Distributions of Publications per Author for Each Time Block .................. 112

4.3 Frequency Distribution of the Intensity of Ties in the Global Network ...... 130

4.4 Distributions of Unique Collaborators per Author for Each Time Block.... 132

4.5 Distribution of Components for Each Time Block ................................... 138

4.6 Distribution of Bi-Components for Each Time Block ............................... 139

4.7 Frequency Distribution of all K-cores All, 2000-2004, and 2005-In Press . 142

4.8 Distribution of Components after Three-Clique Assessment ..................... 144

4.9 Frequency Distribution of Centrality Scores ........................................ 157

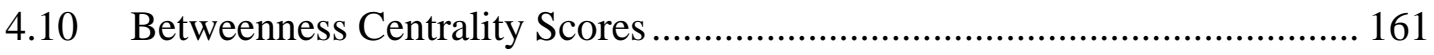

4.11 Correlations for Emergent Themes and Centrality Measures..................... 163

4.12 Frequency Distribution of Bridges per Vertex........................................ 166 


\section{LIST OF FIGURES}

Figure

1.1 Coauthorship Network Characterized by a Model of Scientific Stars ............... 21

1.2 Coauthorship Network Characterized by a Small-World Model...................... 22

1.3 Coauthorship Network Characterized by a Model of High Cohesion ............... 22

4.1 Global Network Illustration of Sport Management Unique Ties...................... 116

4.2 Global Network Illustration of Coauthorship Tie Strengths ............................ 117

4.3 Global Network Structure of Intensity of Ties Prior to 1985 .......................... 118

4.4 Global Network Structure of Intensity of Ties 1985-1989 ............................ 118

4.5 Global Network Structure of Intensity of Ties 1990-1994 ............................. 119

4.6 Global Network Structure of Intensity of Ties 1995-2000 ............................. 119

4.7 Global Network Structure of Intensity of Ties 2000-2004 ........................... 120

4.8 Global Network Structure of Intensity of Ties 2005-In Press ......................... 120

4.9 Illustration of the K-Core Groups for the Main Component ........................... 141

4.10 Subgraph of 3-Clique Overlaps of Main Component for All Time Blocks....... 144

4.11 K-cores 7-9 in the Derived Components from the 3-Clique Analysis............... 146

4.12 Intensity of Ties of K-Cores 7-9 in Components of the 3-Clique Analysis....... 148

4.13 Visual Image of Partitioned Centrality Scores of the Largest Component........ 158

4.14 Visual Image of Betweenness Centrality for Largest Component.................... 161 


\section{GLOSSARY OF NETWORK TERMS}

Bicomponent--components that require there to be at least two independent paths connecting pairs of actors

Betweenness Centrality - the extent to which an actor is needed as a link in the chains of contacts that keep the network connected

Bridge-any line whose removal increases the number of components

Centrality - the extent to which a node/actor is involved with other actors in the network

Centralization - the extent to which there is a clear boundary between the center and the periphery of the network

Clique — a maximally connected subgraph of a minimum of three vertices

Closeness Centrality - how close an actor is to all the other actors in the set measured by the number of other vertices divided by the sum of all distances between the vertex and all others

Cluster --Although cliques and clusters are sometimes used interchangeably in the literature, there is a distinct difference between the two terms. Cliques are defined by very specific mathematical properties and are considered first and foremost properties of the concrete structure of ties between actors. Clusters, on the other hand, are defined in terms of their contiguity in the diagram and separation from other clusters. That is, not all clusters will be nodes that are equally close together but rather represent separations of the similarities of the attributes (either structural or simple attribute properties) possessed by actors.

Component -- a connected subgraph in which all of its vertices are linked by a path or paths to one another but have no ties outside the subgraph

Degree - the number of lines incident with a node in a graph; represents the number of ties an actor in the system has to other actors 
Density - the percentage of lines present in the network relative to the total possible number of lines that could be present given the number of actors

Edge - a line, tie or relation drawn between two nodes

Geodesic - the shortest path between two vertices

Isolates - Vertices that have a degree of 0 meaning that they that have no connections to other vertices

$k$-core-a maximal subgraph in which each vertex has at least a degree of $k$

Node - a point or vertex representing an actor in the system in a graph

Partition-a subset or cluster of a network's vertices

Path - a walk of any length in which no vertex between the first and last of the vertex of the walk occurs more than once

Reachability - the extent to which it is possible to reach one node from another node and serves as a more effective indicator of network connectivity than density

Small World Model — a model in which the level of local clustering is high but the average number of connections need to reach each other is low

Star Model — a model in which one actor is tied to a number of people who are not hihgly connected to each other; a model of preferential attachment

Structural Cohesion - a model in which there are multiple paths passing through different actors such thatif any one actor were to be removed, alternative paths remain that maintain connectivity 
Sub-graph - any collection of points selected from the whole graph of a network with the lines connecting the points still in tact

Vertex — node or point representing an actor

Walk - the presence of any connectivity pattern that allows you to travel from vertex to another 


\section{CHAPTER 1}

\section{INTRODUCTION}

As the recipient of the 2004 Earl F. Zeigler Award, Dr. Wendy Frisby eloquently challenged scholars in the field of sport management to embrace critical social science as a lens of inquiry (Frisby, 2005). She contended that by revealing the bad and ugly sides of sport, researchers can expose new ways for sport managers to promote the good aspects of sport. Conveying concern that research in the field of sport management tends to be overly focused on the same theories and paradigms as well as applied to only a narrow scope of sport domains, Frisby stressed the need for and value in utilizing diverse topics and approaches to enhance our understanding.

Frisby has not been alone in her assertions. In fact, a number of sport management researchers, including several other previous Zeigler award winners, have raised similar issues (e.g., Chalip, 2006; Pitts, 2001; Slack, 1996; Slack, 1998). Some scholars have expressed concerns about the lack of domain diversity and limited range of topics being explored (Pitts, 2001; Slack, 1998). Others have criticized the arduous usage of survey methods and lamented that the research subjects in sport management studies are excessively homogeneous in nature (Olafson, 1995). Moreover, scholars have raised concerns about the legitimacy of the field in the realm of academia in general (e.g., Chalip, 2006; Slack, 1998). 
Incidentally, a recent special issue in the Journal of Sport Management reflected on the constrained idea space (i.e., content and diversity of knowledge circulating) in the field and focused on "expanding the horizons” of sport management research through critical and innovative approaches. In the introduction of the issue, Amis and Silk (2005) expressed their hope to "raise questions, provide an opportunity for thoughtful reflexivity, [and] offer a range of counter-hegemonic approaches” (p. 355). In addition, by recognizing, and in essence advocating for the alternative methods presented, Amis and Silk (2005) sought to "aid the power of those in the academy to apply research so that it impacts, and is meaningful to, the various communities that sport management has the potential to touch” (p. 355). In many regards, the focus and content of this special issue demonstrated an emerging recognition that there is a necessity for looking beyond the dominant mainstream frameworks and approaches in order to promote a more inclusive culture for the generation of knowledge in the field.

Such critical reflection on the idea space and research practices of academic disciplines is not limited to the field of sport management. Prompted by critical theory and postmodernism, analytical reflection on the state and practices of disciplines is now common among many branches of the physical and social sciences. As a result of such critical analyses, many of the longstanding beliefs and values pertaining to arguments of what makes scientific research scientific have been challenged.

At the core of these deliberations are deeply-rooted philosophical questions such as what constitutes justifiable knowledge; in what ways is knowledge recognized and understood; and how is knowledge stored, distributed, and put into use? Likewise, 
questions about the generation of knowledge claims and specifically how people can go about producing value-free facts about the world have spurned still further debate (Glesne, 2006). In fact, a number of perspectives are challenging the nature and practices of science in their very essences.

The foundations of many of these disputes stem from the distinction between positivist and constructivist views of the world. Positivists generally believe that there is one true reality and that people can come to know and understand this "true reality" through objective (i.e., unbiased and impartial) means of experience and inquiry (Glesne, 2006; Green \& Stinson, 1999; Lather, 1986). Constructivists, on the other hand, tend to believe that reality is socially constructed, and that there may not necessarily be one truth of reality, but rather many truths. That is, people individually construct reality based upon social processes, structural constraints and opportunities, and their personal attributes. In this regard, subjectivity in knowledge and understandings of the world are considered inevitable (Glesne, 2006; Green \& Stinson, 1999; Lather, 1986).

Grounded firmly in a positivist framework, the traditional culture of scientific practices sought objectivity in research ventures. Historically, the foundations of scientific endeavors centered on the belief that a cumulative body of knowledge based upon value-free measures can lead to an understanding of how the world works (Glesne, 2006). However, contentions raised by Kuhn (1962; 1970) and a number of other scholars shed light on the structural and political forces that play into scientists' activities; essentially countering any semblance of absolute objectivity.

A trained theoretical physicist, Kuhn established philosophical conceptions about how history, power, and social relations inform and shape the practices of science. His 
seminal work, The Structure of Scientific Revolutions, is often credited as an instigating force driving a philosophical re-evaluation of scientific views and practices (Glesne, 2006).

Another monumental component of Kuhn's (1960; 1972) work was his coining of the term “paradigm.” Though much debate and ambiguity surrounds Kuhn's exact contextual meaning of the word, Usher (1996) defined paradigms as "frameworks that function as maps or guides for scientific communities, determining important problems of issues for its members to address and defining acceptable theories or explanations, methods, and techniques to solve defined problems” (p. 15). Substantively, Kuhn used the notion of paradigms to convey the shifting of ideas and practices that take place in a cycle of historically, politically, and socially informed scientific activities.

As a result of Kuhn's and other scholars' work, a line of research emerged attempting to understand the nature of knowledge and scientific practices in a "scientific manner” (Dickson, 1997, p. 334). Referred to as the sociology of science and the sociology of scientific knowledge (SSK), researchers in these areas are concerned with such topics as: 1) the interrelationships between science and other social institutions, 2) science as a social system (i.e., scientific ethos and norms of governance), 3) the characteristics of scientific knowledge and practice that bring about change, and 4) the way sciences use the scientific literature (e.g., citation patterns and classics vs. exemplars) (Crane, 1988).

In providing an overview of these social factors related to the construction of knowledge, Crane (1988) articulated the benefit of synthesizing all of the perspectives. Specifically, Crane (1988) expressed the need for an understanding of how social systems 
of scientific communities influence the development of ideas relative to contexts that bring about discovery and the nature of the scientific literature. Indeed, in more recent years, greater attention has focused toward understanding how knowledge is created, transmitted, used, and stored within and across scientific disciplines (e.g., Collins, 1998; Daipha, 2001, Ennis, 1992).

Research on Social Structures and Scientific Knowledge

As sociological studies of science and knowledge have consistently demonstrated, people influence the beliefs of others with whom they are socially connected, and the beliefs a person holds are largely related to the people with whom they interact (e.g., Kuhn, 1970; Martin, 2002; Moody, 2004). Thus, in considering social influence in knowledge creation and diffusion, understanding the idea space of a discipline requires not only a conception of the ideas and knowledge produced in the discipline, but also an assessment of the social relationships between the people and institutional structures that are producing, practicing, and transferring the ideas (Crane, 1988). As such, recent approaches for studying the state of scientific disciplines have particularly focused on how social structures within scientific communities create opportunities for and impose constraints on scientific knowledge productivity and growth (e.g., Ennis, 1992; Freeman, 2004; Friedkin, 1998).

Researchers have employed a variety of empirical approaches to evaluate the idea spaces and social structural features of scientific disciplines. For example, ethnographic accounts, case studies, document and discourse analyses, and survey methods have all been used in exploratory studies related to specific academic fields (Moody, 2004). 
Accordingly, several themes emerged from such investigations including increases in collaboration rates (Price, 1986), theoretical fragmentation (Abbott, 2000; Collins, 1998), disproportionate production and recognition of the same few scientific "stars” (Merton, 1968), and the balkanization of researchers in a field (Crane, 1988; Daipha, 2001; Ennis, 1992).

In addition to the previously mentioned approaches, recent work in the study of sociological factors in scientific disciplines has incorporated an emerging methodological perspective: social network theory and analysis (e.g., Freeman, 2004; Moody, 2004). While it has long been theoretically posited that scientists greatly influence each other's work (e.g., Kuhn, 1970, Merton, 1968), and that networks of collaboration shape the idea space of a discipline (Crane, 1988; Kuhn, 1970), empirical evidence supporting these notions has been largely derived from ethnographic accounts (e.g., Collins, 1998; OwenSmith, 2001) and smaller groups in specific settings (Friedkin, 1998). However, as Moody (2004) emphasized: “Although we might expect the link between networks and ideas to be strongest in small groups, a logical extension suggests that long-term trends in scientific work might depend on the broader pattern of disciplinary social networks” (p. 213). As such, Moody (2004) used a social network approach to study collaboration trends and social structural features of sociology as a scientific discipline.

\section{A Social Network Perspective}

A social network perspective has become one of the most riveting and helpful means for analyzing the social structures and processes of scientific communities (e.g., Crane, 1988; Freeman, 2004; Fuchs \& Ward, 1994; Moody, 2004). In addition to the 
growing interest in using a network approach for studying the sociology of scientific knowledge, the integration of social network techniques has risen in a number of fields of inquiry and been applied to a diverse array of topics. Although a lot of the basic theory and methodological techniques have been around for decades, social network analysis has only started to gain popular acceptance in more recent years (Scott, 2001).

One reason for the sudden growth in interest is the powerful implications that network techniques render in assessing the relationships among social entities while at the same time providing concrete depictions of the patterns and implications of such relationships. By utilizing relationships as the primary unit of analysis, in contrast to the conventional approach of focusing on attributes, network theories and analyses provide leverage and insight for philosophically, theoretically, and empirically investigating a diverse range of questions (Wasserman \& Faust, 1994). To convey the uniqueness and power of network analytic techniques, it is useful to engage in a discussion about where network methods fall into the understandings and practices of conventional research methods.

"Quantitative” and "qualitative” are both large-scale umbrella terms commonly used to describe research methods. While the two terms share some foundational similarities, more often than not they are pitted against one another as binary opposites (Abbott, 2001). Fundamentally, although it is less often discussed in the literature, one could argue that "network analysis" is also a distinct methodological umbrella term much like the terms "qualitative" and "quantitative" (Kilduff \& Tsai, 2003).

In spite of the fact that each of the three approaches constitutes unique ways of investigating the realities that exist, and provide different tools for analyzing and 
interpreting the phenomena under investigation, all of them implement similar activities in their research processes. In general, all three explicitly or implicitly rely on theory, are concerned with rigor and thoroughness of study, raise questions and/or pose a problem, collect and analyze data, and present results.

Nevertheless, it is vital to see these terms as mere figurehead classifications, as the methodological assumptions and approaches classified under even their own umbrellas can be quite different from one another in their scope, assumptions, and procedures. One of the principal distinctions between quantitative, qualitative, and network analytic approaches is the fact that each invokes certain tendencies toward an assortment of metaphysical views. More specifically, each approach possesses relatively specific assumptions that guide the research process.

One useful way of thinking about network analytic techniques is that they are constructive approaches intertwining quantitative and qualitative philosophies, data collection, analysis techniques, and interpretation practices. According to Brieger (2004), “The distinction between 'quantitative' and 'qualitative' approaches to data analysis is called into question by network analysis” (p. 511). Although, many of the network analytic techniques integrate a consideration of statistical relationships among variables much like mainstream "quantitative” methods, the variables of interest would tend to fall under more of a "qualitative” categorization (Brieger, 2004; Scott, 2001). As an emphasis to this point, Scott (2001) contended that the methods most appropriate for assessing relations as the properties of systems of agents are those of network analytic techniques 
articulating that, "While it is, of course, possible to undertake quantitative and statistical counts of relations, network analysis consists of a body of qualitative measures of network structure” (p.3).

In many conventional quantitative analyses, underlying assumptions of individuality and independence for units (be they persons, groups, events, etc.) are presumed. Consequently, researchers commonly aggregate and cross-analyze individual units and attributes to tease out the separate and conjoint effects of multiple variables. In many qualitative approaches to inquiry, such as critical theory, deeply-rooted structural holisms are often viewed as forces leading to certain actions (Degenne \& Forse, 1999). As such, contextual and emergent research designs are employed to attempt to understand how these deep-seated structural norms constrain human behavior (Glesne, 2006).

Thus, in essence, the tendency is to embrace one of two assumptions for social behavior: 1) individual characteristics as the driving force behind action--an undersocialized perspective according to Granovetter (1992), or 2) social norms as the driving force behind action—an oversocialized perspective according to Granovetter (1992). Studies using the former philosophy discuss things such as demographic variables (sex, race, education, age, etc.) and psychological variables (personality, attitude and affect) as explanatory schema for social behavior, while studies adopting the latter approach view societal pressures as deterministic forces driving action. Incidentally, a general limitation is shared by both approaches. Neither of them adequately captures the duality between individuals and the groups to which they belong (Breiger, 1974; Wellman, 1988). 
As such, one of the major benefits of using network analytic techniques is that a researcher can investigate how actors are located or "embedded" in the overall network as well as holistically assess the patterns of relationships among all of the actors (Wasserman \& Faust, 1994). Thus, rather than focusing on how an actor's attributes are related to other actors' attributes, network analysts are more concerned with seeing a structure of relations. This difference in emphasis is consequential for the choices a researcher makes in deciding on research design, sampling techniques, measurement, and analysis.

Supporters of network analysis have identified several other distinctive features that differentiate it from traditional analyses: 1) network research is amenable to multiple levels of analysis thus providing some fluidity between micro-meso-macro linkages (Kilduff \& Tsai, 2003); 2) network research can integrate quantitative, qualitative and graphical data producing a more thorough and richer analysis of the phenomena (Scott, 2001); and 3) network approaches allow for a more concrete vitality of difficult to define constructs (Emirbayer, 1997; Wasserman \& Faust, 1994). Innately, these differences provide advantages that can greatly facilitate measurability and interpretation which can ultimately reveal compelling and innovative discoveries (Emirbayer, 1997; Wasserman \& Faust, 1994). In addition, network approaches can be used to complement conventional approaches to provide a more robust understanding of the phenomena of interest (Kilduff \& Tsai, 2003). 
Social Network Analysis and the Field of Sport Management

Incidentally, studies using social network analysis have yet to be explored in any realm of the discipline of sport management. For example, while the idea of social influence is often implied or referred to in sport management literature on consumer behavior, (e.g. Laverie \& Arnett, 2000; Funk and James, 2004; James, 2001), conceptual and empirical studies specifically integrating the role others play in influencing others' behaviors and attitudes have been fairly scarce. Although consumer behavior is used as the primary example here, the same critique can be applied to many of the topics of interest in the field.

Much of the traditional research in sport management has focused on identifying and measuring the personal attributes, attitudes and perceptions of individuals. Likewise, individuals conforming to social norms (i.e. gender, race or ethnic variables) are often used as explanatory elements for many studies in the field. Thus, on the one hand, individuals are often assumed to be acting in complete isolation of one another, while on the other hand, individuals are construed as strong conformers to social norms. With these perspectives, individuals’ behaviors are automatically assumed to be a relative function of conscious rational choice, random interaction, or socially constraining factors. However, people do not operate in social or environmental vacuums and are driven to action by both conscious and subconscious motives. Nevertheless, decision-making, attitudinal formation, and other processes of interest in sport management research (and other fields) often embrace one of these two extreme approaches taking on either an oversocialized or an undersocialized view of the world. 
Conceivably, one major reason for the gaps in the literature is that the capabilities of traditional research methods have not allowed for adequate integration of social influence into measurement and interpretation techniques (Scott, 2001; Wellman \& Berkowitz, 1988). Although sport management studies often describe philosophical and paradigmatic approaches that incorporate social interaction and processes as essential components, conventional analytical instruments have been insufficient for testing a social reality of such complexity. However, by providing analytical tools for overturning some of the undersocialized, and oversocialized limitations of the more conventional research methods, social network techniques may indeed prove to be a valuable methodological approach for investigating even more diverse topics and domains in the field of sport management.

\section{STATEMENT OF THE PROBLEM}

As a relatively young academic discipline, many scholars have noted the astounding growth of the field of sport management over the past couple of decades (e.g., Chalip, 2006, Costa, 2005; Weese, 2002). In fact, according to Chelladurai (2005) with over 200 universities in North America offering degree programs at the bachelor's, master's, and doctorate levels, sport management "is among the fastest growing areas of study in American universities” (p.5). However, despite its rapid growth and development, the field of sport management has not escaped the "growing pains" that often arise as new fields emerge (Amis \& Silk, 2005; Chalip, 2006; Costa, 2005).

Among these growing pains include ongoing debates concerning such issues as the definition and boundaries of the field (e.g., Costa, 2005, Pitts, 2001), the role of sport 
management as an academic discipline (e.g., Costa, 2005; Slack, 1998), and the appropriate research topics, goals, and approaches that should be pursued and implemented (e.g., Boucher, 1998; Chalip, 2006, Costa, 2005, Pitts, 2001). Costa (2005) articulated that the varied perspectives pertaining to these issues "are both a symptom and an outcome of the fact that sport management is still a young discipline” (p. 118). Nevertheless, as Chalip (2006) expressed, there are advantages to being a younger discipline; specifically the “opportunity to build the discipline’s foundation and to shape its future” (p.3).

Indeed, in the spirit of Kuhnian notions of scientific revolutions, scholars in the field of sport management have started to recognize and acknowledge how working through these growing pains can inherently facilitate the growth and maturation of our emerging field (e.g., Amis \& Silk, 2005; Chalip, 2006; Costa, 2005). As Costa (2005) expressed, “discussions and debates of this kind are useful for the advance of scholarly inquiry because they help to clarify needs, assumptions, possibilities, goals, and directions” (p. 118). Moreover, Costa (2005) contended that specific empirical and systematic inquiry related to the parameters and implications of such debates are conducive for advancing the scholarly work in the field.

As such, Costa (2005) argued that since the advancement of knowledge is a “collective endeavor,” empirical analyses examining the current status and future directions of the field should be "collective in nature” (p. 119). While this is an important argument, and indeed one that Costa fruitfully engaged in, the collective nature of the advancement of knowledge can be looked at from a different angle. Specifically, how is the advancement of knowledge in our field being collectively pursued? 
For example, positivistic, quantitative methodologies have historically served as the dominant approaches for research endeavors in the field of sport management (Gratton \& Jones, 2004; Skinner \& Edwards, 2005). Over the last decade or so, however, more and more scholars in a variety of fields have asserted the need for the embracement of alternative worldviews and more diverse methodological toolboxes to investigate questions about the social world (e.g., Denzin \& Lincoln, 2003; Glesne, 2006). Indeed, this trend toward "expanding the horizons" and extending the idea space of a scientific discipline through the promotion of alternative approaches to inquiry has made its way into the sport management literature (e.g., Amis \& Silk, 2005).

Nevertheless, although in recent years researchers in the field of sport management have made calls for, and in many ways, great strides toward the integration of more postpositivistic, innovative approaches (e.g., Frisby, 2005), the rate of embracement suggests a culture of guarded optimism at best toward these new approaches (Skinner \& Edwards, 2005). As Amis and Silk (2005) articulated, There is little doubt that some related disciplines—such as education, cultural studies, leisure studies, and the sociology of sport—have progressed more rapidly than sport management in their acknowledgement of the value of different ideological, epistemological, and methodological approaches (p. 356).

In taking a critical look at this fact, the question thus arises, are there specific features of the field of sport management that impede the ability of innovative ideas from being integrated into the idea space and practices of the discipline?

With the incorporation of postmodern views into the field of sport management, scholars have emphasized "the need to understand sport management in its wider, 
political, economic, and ideological context and be concerned with exposing patterns of inequality and intervening in local communities” (Amis \& Silk, 2005, p. 357). While this is of course important in a grander sociological sense, it is also important to recognize the significance that these needs hold even within the practices of our own community-the community of scientists in the field of sport management. That is, researchers in the field of sport management need to be concerned with the political, economic, and ideological patterns of inequality that may exist in terms of scholarly interactions with one another.

Certainly, the dream of an open-minded and diverse culture of scholarship is not an uncommon goal for researchers in the field. As Amis and Slack (2005) expressed, "In keeping with a field that is inherently multidisciplinary in nature, we envision an academic landscape that is not dominated by any single overarching metanarrative that marginalizes and obfuscates alternative approaches” (Amis \& Silk, 2005, p. 358). However, dreaming and calling for a more inclusive idea space and actually possessing a more inclusive idea space are not necessarily one and the same. Consequently, we need to search for, identify, and attempt to overcome any inequalities that exist which can potentially impede the generation and spread of knowledge within our own scientific community.

To illustrate, let us say we are trying to introduce a new methodological paradigm, into the field of sport management's scientific activities and literature. What factors might inhibit the spread of this new paradigm among the scholars in the field? Intuitively, we can assume that the attributes of the paradigm itself might lend it to either a quick embracement or rejection as a methodological approach of use, value, and accessibility. We might also consider the abilities of the scholars in the field in such 
regards as: 1) the ability of those trying to push for the new paradigm to do so in a convincing matter; 2) the ability of those reviewing the ideas to comprehend and see the benefits of the new paradigm; and 3) the ability of other scholars in the field to understand and implement the new paradigm in applicable and relevant ways.

While these are all important considerations, a more holistic approach might lead one to question what factors affect the spread of anything through a system of actors. In this regard, epidemiological models of disease spread and the literature on the diffusion of innovations offer some unique insight into how one might go about envisioning and predicting the transmission of ideas throughout a population of scholars (Rogers, 2003; Watts, 2000). With a specific focus on the social influence of others, and in particular social networks, this is precisely the route that scholars studying the generation and construction of knowledge within scientific communities are starting to integrate (e.g., Crane, 1988; Friedkin, 1998; Freeman, 2004). In fact, with the ability to illuminate patterns of relations and concrete social structures of interaction, social network analysis is fast becoming one of the most powerful approaches for studying the diffusion of ideas in scientific disciplines.

One advantage of adopting a network perspective is the ability for a researcher to investigate and explain phenomena relative to dynamic relationships and unfolding processes. Thus, rather than only asking ourselves where the current state of the field of sport management stands, significant insight can be gained by focusing on attempting to understand how the current voids and dominant forms arose in the first place. Essentially, moving from simply identifying what the dominant idea spaces in the field currently entail and where they should go from here, we can take a historical look at how, when, 
and from whom the intellectual foundations of the field emerged. With these ideas in mind, an exploratory investigation into the underlying sources and forces that have helped establish the structure of the discipline can provide valuable insight into identifying gaps and opportunities for the state of the field to be improved.

\section{PURPOSE OF THE STUDY}

Amis and Silk (2005) lamented, “Too often our work in sport management has been presented as neutral and value free, with little regard for the historical, social, political, and cultural context in which the work takes place” (Amis \& Slack, 2005, p. 357). As the North American Society for Sport Management (NASSM) is fast approaching the $25^{\text {th }}$ anniversary of its inception, the intent of this paper is to critically review and empirically explore the social structure of scientific endeavors in the field of sport management in North America. Integrating a network perspective, the ideas presented in this study offer a unique and compelling approach toward identifying the social structure and potentially hegemonic tendencies shaping the discipline. The findings of this study are tied to the theoretical implications these structural characteristics may hold for the idea space as well as the growth and progression of knowledge claims in the field.

In addition, a secondary thrust of this study, was essentially to introduce social network analysis as a new and promising lens for exploring facets of human behavior to the field of sport management. The ideas presented are not to suggest that the traditional approaches and methodologies should be abandoned. Rather, the propositions are meant 
to encourage researchers to integrate and synthesize traditional approaches and methodologies with network approaches for a greater understanding of the multi-faceted dimensions and topics of interest to sport management scholars.

By incorporating some of the techniques of social network analysis into the process of a critical investigation of the underlying structures of the field, it is the authors' hope to insightfully delineate how some of the basic concepts and implications of network analytic techniques can provide an opportunity to explore more diverse and robust research questions in the future. Moreover, concurrent with the calls for critical reflection, the questions being investigated in this study offer ample opportunity to challenge and strive to improve the idea space of the field. After all, as Amis and Silk (2005) expressed, "a healthy sport management is surely one that is constantly questioning and challenging itself” (p. 355).

\section{THEORETICAL PREMISES AND RESEARCH QUESTIONS}

The theoretical grounding for this study was based upon the ontological and epistemological premises related to the social construction of knowledge. Further, the data was collected and explored through the lens of a social network perspective. In particular, this study was developed to investigate the underlying social structure of the discipline of sport management and tie the results to potential implications for the generation and spread of knowledge claims in the field. More specifically, the purpose of the study was essentially to explore the question: What do the patterns of scholarly interaction hold for the field of sport management's idea space? 
It has been suggested that some of the most important indicators of social organization in research areas include informal discussions of pertinent research topics, relationships between advisors and doctoral students, and collaboration on publications (Crane, 1988). Through these types of interactions, the sharing and negotiating of ideas is likely to lead to a relative consensus in terms of attitudes and beliefs about the ontological, epistemological, and methodological techniques that are accepted as appropriate as well opinions about the topics that should be explored. Thus, scientists who collaborate are likely to share similar ideas, employ analogous research techniques, and significantly influence each others’ work.

Likewise, studies have suggested that there is a direct linkage between patterns of social interaction, the structure of idea spaces, and the technical practices of a scientific discipline (e.g., Friedkin, 1998; Collins, 1998; Moody, 2004). Further, researchers have contended that if a group of individuals exchange ideas, ask similar research questions, and follow implicit and explicit rules of systematic data collection and analysis, then a consensus of coherent and recognized idea systems should be related to the social and structural cohesiveness of the discipline's underlying network structure (Friedkin, 1998; Moody, 2004). Therefore, according to Moody (2004), if idea spaces and belief structures critically depend on the shape of the underlying social networks, we can draw hypotheses about relationships between the structure of interaction networks and the idea space of a discipline.

As Crane (1988) contended, one of the main channels for scientific influence is through collaboration with others on research projects. Accordingly, Moody (2004) articulated, "If the collaboration network shapes commitment to particular ways of doing 
science, then identifying systematic differences in who collaborates will identify key differences in those exposed to the information and ideas flowing through the collaboration network” (p. 222). In studying such social networks, one of the most visible and tangible manifestations of social interaction in an academic discipline are the coauthorship patterns of research publications (Crane, 1988; Moody, 2004). As such, an empirical assessment of the formal coauthorship relationships is both a feasible and useful means of exploring the underlying social structure of an academic discipline.

Thus, one primary focus of this study was to construct networks of the coauthorship patterns in the field of sport management. In essence, the research design for this study was in many ways similar to Moody’s (2004) exploration of the discipline of sociology's underlying collaboration network structure. The data collected was used to construct coauthorship networks in the discipline and describe trends in the sport management collaboration network over time.

The overriding structural question driving this study was: What is the structure of coauthorship patterns in the field? Secondarily, the outcome of the network structures were coded into network properties to look for themes related to variables that are perhaps correlated with scholars' locations within the overall network. Finally, the networks constructed were viewed as conduits for diffusion to explore the question: What does the structure implicate for the transfer of ideas throughout the network?

Since no prior research on the collaboration patterns in the field of sport management has been conducted, it was difficult for testable statements to be generated. Consequently, the analysis stage employs exploratory social network analysis only. In other words, no specific hypotheses about the structure of the network were tested. 
Instead, the researchers sought to develop themes about meaningful patterns related to the social structure of the obtained network with the goal of establishing a foundation for future studies.

The derived network structure was discussed relative to three competing theoretical models of scientific knowledge growth and disciplinary structures: 1) a preferential attachment model where the structure is reliant upon a few "star" producers, 2) a small-world model where specialty areas cluster into distinct social groups, and 3) a structurally cohesive model that suggests a broad over-arching connectivity among many of the actors in the network (Moody, 2004). As each of these models posits unique theoretical and conceptual implications for the idea space and practices of a scientific discipline, the structure of collaboration patterns in sport management were ultimately tied to claims about scientific practices. Overviews of the three models are described in depth in Chapter 2. For now, Figures 1.1, 1.2, and 1.3 provide graphical depictions of each of these models. The points, or nodes, are used to represent scientists in a field and the lines, or edges, between the points represent connections (e.g., collaborative interactions) between any two scientists.

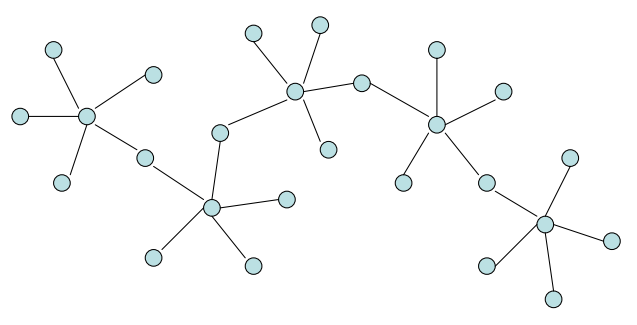

Figure 1.1 Coauthorship Network Characterized by a Model of Scientific Stars 


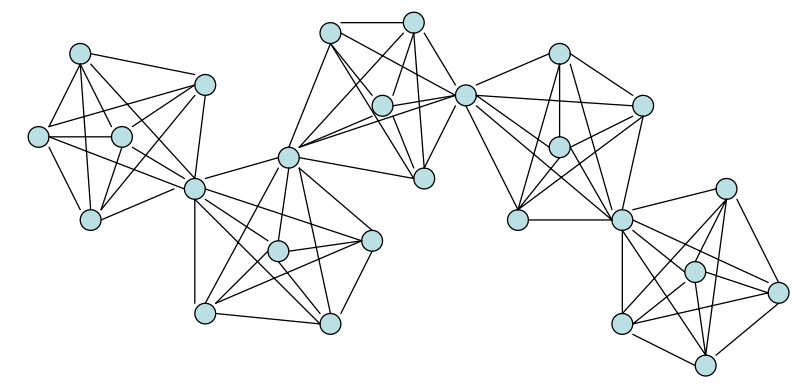

Figure 1.2 Coauthorship Network Characterized by a Small-World Model

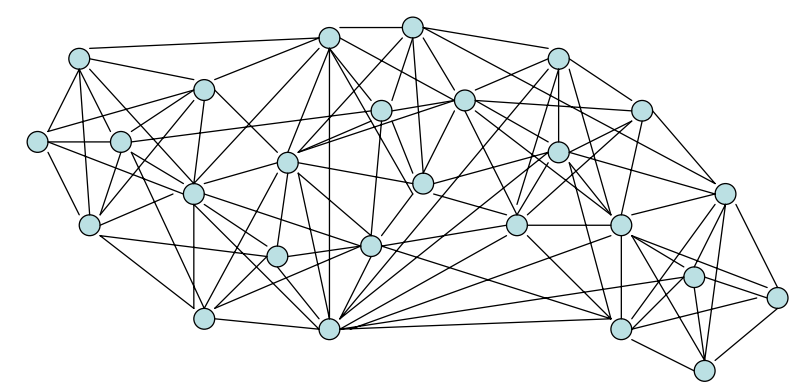

Figure 1.3 Coauthorship Network Characterized by a Model of High Structural Cohesion

\section{$\underline{\text { Structural Considerations }}$}

The models presented above are of course in many ways unrealistic. That is, a network obtained based upon real-life activity should not be expected to form such structurally consistent patterns throughout. Thus, the network obtained actually exhibits 
patterns pertaining to all three models in that parts of the network are comprised of structural stars, parts of the network are highly clustered and exhibit "small-world" qualities, and a number of areas consist of relatively large, cohesive subgroups. As such, in Chapter 5, the manifested patterns of the network are tied to several of the implications suggested by literature related to the diffusion of innovations which are outlined in the upcoming paragraphs.

\section{Network Distance}

Under the premises of network diffusion models, it is assumed that social relations and contacts from one person to another are conduits for social contagion and influence (de Nooy et al., 2005). Therefore, a necessary requirement is that people who have not yet adopted an innovation must come into contact with someone who is either an innovator or has already adopted the innovation. From a structural standpoint, this essentially means that an actor must not only be present in the network but actually be connected either directly or through intermediaries to innovators or adopters for networkcontagion to be possible (although it is possible they may adopt through a different source such as the media).

Beyond connectivity, a secondary consideration is how many intermediaries an innovation must go through to reach a potential adopter. According to Moody and Leahey (2006), given simple connectivity, innovations spread over a network in inverse relation to the average distance between nodes (i.e., actors). In other words, the more 
intermediaries an innovation must pass through to reach a potential adopter, the longer the time it will take and the lower the likelihood the innovation will ever reach the potential adopter.

This concept is explored through various measures of connectivity in Chapter 4 and ultimately tied to network centrality measures. In essence, network distance can assess, and in many ways help predict, how diffusion through a network might be affected depending on the location of an innovator or initial adopter. Thus, under these premises we can tie ideas about what locations in a network are the most ideal in terms of spreading innovations throughout the sport management coauthorship network.

\section{Network Clustering}

In real-world networks (i.e., networks constructed from data and not through random procedures), relations tend to cluster or clump around certain features. For example, geographic locations can significantly influence the people with whom you interact. According to Moody and Leahey (2006), clustering can make diffusion through a network "lumpy" because while an innovation can diffuse fairly easily within the clusters, it can be dependent on a certain person or relationship that serves as a bridge to connect two or more clusters. Hence, generally as network clustering increases, diffusion rates tend to decrease.

The clustering of the nodes in the observed coauthorship structure for the field of sport management is examined in a couple of different ways in Chapter 4. First, we attempted to understand the clustering patterns through clique and k-core assessments as 
we explored the network for cohesive subgroups. Secondarily, we also looked at a measure in Pajek that calculates the clustering coefficients to get a sense of the presence of clusters and clustering patterns in the observed network.

\section{Multiple Paths}

Alternatively to network distances in which longer path lengths decrease the likelihood of transmission, multiple paths (i.e., connectivity patterns that allow you to reach one node from another in more ways than one) can increase the likelihood of diffusion (de Nooy et al., 2005; Moody \& Leahey, 2006). The presence of multiple paths in a network allow information exchange and influence to occur even if a particular node or tie does not contribute to the diffusion (i.e., adopt or get enacted). Moreover, multiple paths can also increase the constraint on an individual to adopt because more of their contacts have already adopted.

In Chapters 4 and 5, we consider several indicators of multiple path presence in the observed network including visualization techniques, clique assessments, and bicomponent extraction. Once again, while these measures cannot provide an indication of who will be the innovators or first adopters, they can provide us with a general sense of the ease with which an innovation can diffuse through the network. In essence, multiple path indicators provide a general sense of structural exposure opportunities.

\section{Timing}

Moody (2000) highlighted the fact that opportunities for contact and thus influence can significantly affect diffusion patterns. For example, Moody and Leahey 
(2006) noted that the lowest possible transmission patterns occur when relations are of short duration and actors are only tied together one time. In addition, real-world networks are almost always dynamic in nature and continually changing. That is to say, new actors are continually being added to the network, others may leave or cease to be active in the network, and the relationships between actors are not always stable across time.

Consequently, all of these are important considerations when assessing the paths engaged and the likelihood for diffusion to occur.

In Chapters 4 and 5 we take such timing issues into consideration in a couple of different ways. One way we examined the connectivity patterns in the network was through an assessment of the strength of ties as calculated by the number of publications a pair of actors produced together. We also took a historical approach by looking at how the network evolved over six designated time blocks: prior to 1985, 1985-1989, 19901994, 1995-1999, 2000-2004, and 2005-In Press.

\section{Thematic Considerations}

While the above mentioned considerations pertained mostly to structural variables that affect the diffusion of innovations in any network, we also wanted to get a general sense of 1) how the patterns of connectivity might have emerged in the first place and 2) identify the presence of potential socio-structural barriers to diffusion within the field of sport management. Consequently, we identified some specific attribute variables that we wanted to use to qualitatively assess the obtained network structure. These included: 1) 
student-advisor and institutional affiliations, 2) years in the field and productivity as calculated by the number of publications an actor has produced, 3) coauthor diversity, and 4) specializations and methodological preferences.

\section{Student-Advisor/Institutional Relationships}

According to Mullins (1973), new fields of research tend to emerge through a diffusion process whereby a certain idea or perspective surfaces at a particular university and students at that university become trained in that area. As the students graduate and move onto other jobs and research projects at other universities, a new crop of students and collaborators are introduced and trained in that perspective. Freeman’s (2004) study on the sociology of social network analysis supported this notion. Hence, it could be argued that exposure to and acceptance of new ideas is inextricably linked to the relationships between doctoral students, their advisors, and the institutions with which they are affiliated.

For the purposes of this study, the following patterns were explored: 1) Is there a reason to believe that there is a relationship between the institution from which a person receives their degree and/or institutions where they carry out their research and with whom they collaborate (i.e., other alumnae/alumni and professors from their degreegranting institution)?; 2) How do advisors' locations in the collaboration network relate to a student's location in the network?; and 3) Does the institution an actor received their doctoral degree seem to be related with location in the network? 


\section{Time and Productivity}

As networks are products of dynamic, unfolding relations, time can significantly influence the relative properties of a collaboration network. In much the same way, time can also significantly impact an individual's location within a collaboration network. For example, the more time a person has spent working in a discipline, the more exposure they have likely had to others' ideas, the more opportunities they have had collaborate with other actors, and the more time they have had to produce publications. As such, time spent in the discipline should be a relatively good predictor of a person's embeddedness in the collaboration network (Moody, 2004). Likewise, the number of publications a researcher has produced should also tend to increase a person's embeddedness in a network. Both time in the network (calculated by the year in which an actor's first publication appears in the network) and productivity (measured by total number of publications) were explored relative to an individual's embeddedness in the sport management collaboration network.

\section{Coauthor Diversity}

As defined by Moody (2004), coauthor diversity measures the extent to which a person collaborates with different coauthors, relative to their opportunity to coauthor with others. Moody (2004) suggested this variable can be calculated as the number of observed coauthors divided by the maximum possible number of coauthors given the number of papers published and the number of authors on each paper. Given that the more diverse coauthorships an actor possesses should indicate higher overall connectivity for that individual in the network, an individual's measure of coauthor diversity should 
also be a good predictor of network embeddedness. Thus, coauthor diversity was qualitatively considered for all actors in the network and the relationship between this variable and network embeddedness were explored.

\section{Specializations and Methodological Similarities}

According to Rogers (2003), the nature of flows of communication through interpersonal networks can be strongly related to the concepts of homophily and heterophily. The principle of homophily refers to the degree to which a pair of individuals or a group of individuals who communicate together are similar. The similarities can be related to any number of attributes such as beliefs, educational backgrounds, interests or even structural locations within a network. Contrastingly, heterophily refers to the degree to which pairs or groups of individuals who interact are different in regard to specific attributes.

According to Rogers (2003), homophily in a network (whether it be in terms of structural or personal attributes of actors) can act as an invisible barrier to the diffusion of an innovation in a network. Therefore, we identified two attributes that we thought might be highly related to the patterns of connectivity in the observed network structure. First, we looked at specializations within and outside the field of sports management. We also considered methodological preferences and tendencies in terms of who collaborated with whom and network centrality. 


\section{Alignment of Formal and Informal Representations of Structure}

Defining the structure of a discipline based solely on collaboration patterns is only one of many indicators of interactive activities that constitute the potential for social influences in a scientific discipline. In addition to the formally recorded co-authorship patterns, other social interaction patterns can be layered onto the constructed collaboration network to provide a more robust delineation of the "true" social structure. For example, a researcher's work can be influenced indirectly by the work and authors that they read and cite. Likewise, informal discussions can take place between actors in the network that are highly influential yet no publication or formal record of their relational interaction may ensue. Thus, data was collected to take some of these other forms of interaction and influence into consideration. Time and resources did not allow for a thorough analysis of this data, however, a surface assessment did reveal a few intriguing patterns.

\section{LIMITATIONS AND DELIMITATIONS}

Since network analyses focus on the relations among actors and not individual actors and their attributes, often actors in network studies are not sampled randomly or independently of one another. Brieger (2004) noted that contrary to random samples, network studies generally necessitate full data on the presence or absence of social relations among all, or at best, most of the members of a bounded population. Nevertheless, whole network studies are not always methodologically feasible or analytically appropriate. Those who use them find that they must define the boundaries of a population, and compile a list of all the relations of interest between all of the members 
of the population. Only then, can a researcher employ "a variety of statistical and mathematical techniques to tease out some underlying structural properties of the social systems” (Wellman \& Berkowitz, 1988, p. 26).

Hence, one of the major limitations of any network study is a researcher's determination of boundary specification for the actors to be included in the analysis (Laumann, Marsden, \& Prensky, 1983; Scott, 2001; Wasserman \& Faust, 1994). A second major limitation of network studies relates to the ties a researcher chooses to use to represent the construct or phenomena under investigation. Thus, it is up to the researcher to determine how to define the boundaries and ties of interest based upon theoretical, conceptual and empirical reasoning. As social network analysis embraces many postpositivistic principles, and infers that the world is comprised of networks of networks, it can be reasonably assumed that the actual number of actors in a network may never be concretely determinable. Moreover, the number and types of ties between the actors under investigation may also be too complex and overlapped to fully capture.

As a general overview, the delimitations of this study included the use of a nominal boundary specification derived through a realist actor enumeration of reputational sampling approaches. That is, the network boundaries were determined based upon theoretically and conceptually informed decisions on the part of the researchers and the enumeration of network actors were generated initially through a reputational approach using key informants. In addition, another delimitation of the study entailed the selection of the types of ties to be explored between actors and hence used to represent the underlying social structure. The relationships of interest selected were also determined by the researchers using theoretical, conceptual, and empirical reasoning. 
Finally, as this is a study conceived based upon notions of the social construction of knowledge, it is important to highlight some of the historical, social, and political biases that may have heavily influenced the researchers' approaches for this study. As a doctoral student still in the infancy stage of my life as a researcher, many of the sources, ideas, and interpretations of the conceptual, theoretical, and empirical approaches I uses were likely to be significantly related to the thoughts and practices encouraged by the members of my committee, the training from instructors during my coursework, and the feedback I received from various sources throughout the stages of the dissertation process. In addition, the writing style and methods implemented are likely directly influenced by the institutional policies mandated for doctoral dissertations as well as the political and financial constraints characterized by a student-conducted study. 


\section{CHAPTER 2}

\section{REVIEW OF LITERATURE}

The discussion in this chapter provides an overview and synthesis of the literature on the status of sport management as a discipline, the social construction of knowledge, and research on the sociology of science. Much of the theoretical grounding derived from this review of literature highlights the implications that social influences and collaboration networks have on the scientific activities that are carried out, the acceptance of knowledge claims, and the theoretical coherence among a community of scholars. The premises of the literature presented ultimately support the need for and value in an investigation into the social interactions and underlying social structures of the scientific community of sport management scholars.

The Status of Sport Management as an Academic Field of Study

While the roots and practice of managing sport and physical activities can be traced to ancient history, the specialized field of sport management as an academic discipline in North America has only achieved a status of formalized organization over the past quarter of a century or so (Chelladurai, 2005; Costa, 2005). According to Chelladurai (2005), three significant factors contributed to the elevation of sport 
management as a field of academic status: the development of degree programs, the establishment of professional associations, and the production of scholarly journals. In fact, the field of sport management has experienced exponential growth over the past couple of decades; most likely as a result of these professionalizing catalysts (Chelladurai, 2005; Costa, 2005; Pitts, 2001).

Chelladurai (2005) suggested that the presence of a professional association is indicative of the maturity of a profession as it provides an outlet that brings people together to exchange ideas and develop self-regulatory policies and practices for members in the field. According to Costa (2005), sport management defined itself as a discipline in the mid-1980s "as signaled by the founding of the North American Society for Sport Management (NASSM) in 1985” (p. 117). Pitts (2001) denoted, “Although the construction of sport management as a field of study did not start with the establishment of the NASSM, this association has had a profound influence in its development” (p. 5). Indeed, since its inception, the number of degree programs, peer-reviewed journals and scholarly conferences affiliated with sport management has grown significantly (Costa, 2005; Pitts, 2001).

As specified by the NASSM bylaws, the purpose of the organization is to promote, stimulate, and encourage study, research, scholarly writing and professional development opportunities in the field of sport management. Among other things, members of the organization are encouraged to explore such topics as theoretical and applied facets of management theory including such cross-disciplinary subjects as leadership, ethics, marketing, finance, communications, sociology, legal aspects and professional growth related to contexts in sport. To facilitate these activities, the 
organization's official research journal, the Journal of Sport Management, is dedicated by design to support research both quantitative and qualitative in nature (NASSM, 2005). According to Chelladurai (2005), another key element in a profession's growth and development is the publication of scholarly works and practitioner journals. Pitts (2001) expressed, “A field of study cannot exist without a body of knowledge and literature” (p. 2). Although a number of sport management textbooks and scholarly journals exist, in many regards the literature in the field is still very young (Pitts, 2001). NASSM's Journal of Sport Management published its first issue in January 1987, and since then, a number of academic and practitioner journals such as Sport Marketing Quarterly and NCAA News have also appeared (Chelladurai, 2005; Pitts, 2001).

Pitts (2001) expressed the irony in the fact that “one of the world's oldest professions is one of the world's newest fields of study” (p. 4). With over 200 universities offering degree-granting programs ranging from the undergraduate to doctoral levels, sport management is currently one of the fastest growing areas in North American higher education (Chelladurai, 2005, Pitts, 2001). Incidentally, as fast as the field is growing, the number of qualified professors to teach sport management courses has struggled to keep pace (Pitts, 2001). Since the education of practitioners and future professoriate can have a profound impact on the status and well-being of an emerging academic discipline, the rapid growth of sport management has spurned both excitement and anxiety from leading scholars in the field (Cuneen, 2004; Frisby, 2005; Pitts, 2001).

As Cuneen (2004) articulated, “An examination of past literature addressing sport management reveals the single emerging theme to be that of potential" (p. 2). As such, the founders and leaders in the field are inherently concerned with finding ways to make 
sure the field reaches that potential through quality controls such as program accreditation procedures, continued professional development, peer mentorship, and coherent theoretical development through rigorous research processes (e.g., Cuneen, 2004; Pastore, 2003; Pitt; 2001). Nonetheless, scholars are not in complete agreement on many issues surrounding the goals and future directions the field should pursue.

For example, one of the ongoing debates attracting the attention of scholars is the very definition and boundaries of the field (Costa, 2005). Likewise, Frisby (2005) and other scholars (e.g., Olafson, 1995; Slack, 1998) have challenged the narrow idea space and limited types of methodologies characterizing the field. In addition, a number of scholars have questioned the legitimacy and role of the field of sport management as an academic discipline (e.g., Slack, 1998; Chalip; 2006) As Slack (1998) articulated, while sport management significantly borrows ideas, theories and empirical evidence from other fields of study, very few other academic fields borrow ideas, theories and empirical evidence from sport management. Consequently, Slack argued that researchers need to find ways not only to use existing theory to study sport but to use sport to test and extend existing theory.

To empirically explore the ongoing debates surrounding the status and future directions of the field, Costa (2005) used a Delphi technique to assess leading scholars' perceptions on the current status of sport management research, some of the major debates plaguing the field, ideal directions for the future of research in the field, and the likelihood that these goals can ever be reached. Among the themes that emerged from the study included disputation on such topics as: 1) the essence of the field, 2) the quality of research design and analysis (in terms of methodological diversity and the 
interdisciplinary/disciplinary distinction), 3) theoretical versus applied research orientations; 4) the infrastructure of the discipline (i.e., conferences, scholarly outlets, and research funding); and 5) the diversity of research in the field (e.g., globalization). Along these lines, the results indicated that scholars identified stronger research, more crossdisciplinary research, a greater union between theory and practice, improved infrastructure, and better doctoral training as important objectives toward which the field should strive.

Many scholars in the field of sport management have acknowledged the need for and value in such critical reflection on the status and practices characterizing the field (Chalip, 2006; Costa, 2005; Frisby, 2005). As Costa (2005) expressed, these critiques help explore "who we are, where we want to go, how we should get there, and where the significant points of disputation reside” (p. 118). Furthermore, Costa (2005) contended, "These are pivotal realms of self-exploration for a young field that seeks to establish itself and its relevance” (p. 118).

Incidentally, the critiques and debates challenging the field of sport management are comparable to ongoing deliberations in other fields (Costa, 2005). In fact, an entire line of inquiry exists which is fundamentally geared toward understanding the activities that characterize and shape the generation, acceptance, and transfer of knowledge in scientific disciplines. As such, the next section delves into the conceptual, theoretical, and empirical aspects related to the construction of knowledge in any academic domain. 


\section{The Social Construction of Scientific Knowledge}

Popular culture's depiction of the quintessential scientist, might lead one to believe that scientific discoveries are made by brilliant, isolated individuals working diligently away in some remote laboratory. In this regard, the growth of scientific knowledge is often attributed to the unique and remarkable discoveries made by a few exceptional people. The findings of these “experts” are consequently viewed as neutral and value-free facts about how the world really works. As such, scientific endeavors are fundamentally accepted as a cumulative body of knowledge progressing toward a deeper understanding of the properties of the world.

However, should we implicitly assume that the findings and claims established by these brilliant individuals are unconditionally true? How are we to judge the strength and truth of their knowledge claims? Are certain discoveries more valuable than others based upon the scope of generality and explanatory power proposed? Moreover, how does one distinguish between knowledge claims derived from "scientific" endeavors from those claims produced through "nonscientific" ventures? That is, what distinguishes “scientific” questions, modes of inquiry and interpretation from findings generated through “nonscientific” enterprises?

Modern philosophers have procured the argument that "knowledge is justified true belief" conveying that it is one thing to say that something is true or false and an entirely other to justify why we say it is so (Solomon, 2002, p. 161). As Fraenkel and Wallen (2003) asserted, beliefs and knowledge can be substantiated in a number of different ways such as logic, opinions of others, sensory experiences, observation, and 
suggestions by figures of authority. Nevertheless, much debate surrounds the question of what constitutes evidence that verifies the truth, falsity, and accuracy of any knowledge claim (Schwandt, 2001).

The word "science" is a term that is commonly associated with the practices of research, investigation and study. However, much controversy stems from the determination of what constitutes "science" and "scientific" activities. The conception of science is fundamentally based upon a desire to acquire and organize knowledge claims in systematic, thorough, and transferable manners. Nonetheless, conceived in this way, knowledge claims derived from any sort of systematic and thorough review could potentially be deemed "scientific." For example, art and biology would both characterize a "science" of sorts. In this regard, then, knowledge pursuits in areas ranging from the humanities to quantum physics could be classified as sciences.

According to Homans (1967), "What makes a science are its aims, not its results" (p. 4). Homans contended that science has two main objectives: discovery and explanation. As such, he suggested, "By the first we judge whether it is a science, by the second, how successful a science it is” (p. 7). Under Homans' premises, discovery is the process of stating and testing more or less general relationships between properties of nature. Explanation, then, is the capacity to explicate how and why the propositions about the relationships under given conditions are true. The strength of an explanation is thus judged on its process of showing how empirical findings follow and/or can be deduced from the general propositions. Homans (1967) further articulated:

If it aims at establishing more or less general relationships between properties of nature, when the test of the truth of a relationship lies finally in the data 
themselves, and the data are not wholly manufactured—when nature...still has a chance to say 'No!' - then the subject is a science. By these standards all the social sciences qualify — even history. The humanities do not. Much fiction, for instance, is very true to life, but the standard by which fiction is judged is certainly not in general this kind of truthfulness (p. 4).

\section{The Process of Discovery}

Stemming from the scientific revolution (i.e., historical changes in thought and belief that unfolded in Europe roughly between 1550-1700), the formalization of “scientific” practices were inspired by a desire for learning and objective knowledge acquisition (Calhoun et al., 2002). During the scientific revolution, as Calhoun et al. (2002) described,

Earlier systems for classifying knowledge were often abandoned, particularly where they were based on surface appearance rather than deeper underlying and causally significant relations. In a thousand different fields from astronomy to the study of moths, from the breeding of farm animals to the growth of the human population, the internal structure of human bodies to the way our sensory organs work, early scientists tried to see as objectively as possible, and to search as widely as possible for things to see (p. 1-2).

Ultimately, the development of modern science significantly reshaped peoples' views of the world around them and how that world could come to be known and understood. In 
essence, scientific inquiry invoked a widespread belief that thorough and rigorous empirical study produces the "truest” and most credible claims and understandings about the world (Calhoun et al., 2002).

Fundamentally, two themes permeate all scientific endeavors: 1) a search for "truths" or understandings about the world, and 2) explanations founded on empirical evidence. Many of the conventional views of science envisioned facts and justified knowledge as "mirrors of reality" and that "facts are discovered when scientists follow only the neutral and disinterested leads of Reason and Reality” (Fuchs \& Ward, 1994, p. 485). However, much criticism and debate has surfaced over whether or not value-free, objective facts can ever be established (e.g., Kuhn, 1970). The roots of these deliberations are characterized by deep-seated philosophical questions about the nature of reality, what we can know, and how certain we can be about the things we know.

\section{Overview of Metaphysics}

At the very heart of these debates is the philosophical complexity of defining the word "knowledge." Plaguing some of the greatest minds of history in metaphysical and scientific searches for universal truth(s), centuries of philosophical debate surround the pursuit for a concrete understanding and consensus of the notion of knowledge. Inherently abstract and transcendental in nature, "knowledge” is not an easy construct to pin down and contain with a few simple words. In general, to establish what constitutes knowledge, how it is generated, and how it is transferred scholars often turn to the specification of various metaphysical assumptions. 
Metaphysics is the branch of philosophy that deals with first principles, the theory of being, and the nature of reality (Glesne, 2006; Schwandt, 2001; Solomon, 2002). Fundamentally, metaphysical theories are concerned with defining the things that constitute the world we know and live in. Much of the discussion surrounding the metaphysical debates is founded upon the establishment of various ontological, epistemological, and methodological premises. In general, Schwandt (2001) articulated that there is often a complementary relationship between the ontological, epistemological, and methodological beliefs that one embraces.

Sometimes used synonymously with metaphysics and other times viewed as a component of metaphysics, ontological beliefs are the views a person adopts about the nature of reality and the means by which that reality is constructed (Schwandt, 2001; Solomon, 2002). Ontology is essentially concerned with questions about whether the world and reality exist, and if so, in what forms? (Glesne, 2006). Upon resolving (or at least contemplating) the nature of reality and how it is constructed, one must then consider how it is we can come to recognize what justifies such beliefs about reality to be true and therefore "known." As such, epistemology is the branch of metaphysics comprised of theories about knowledge and how knowledge is justified.

Correspondingly, methodology refers to theories of how inquiry for knowledge acquisition should proceed (Schwandt, 2001; Solomon, 2002). Schwandt (2001) expressed that methodologies serve as a sort of conduit between the metaphysical underpinnings pertaining to the nature of knowledge and the actual practices of knowledge generation. They fundamentally explicate and define the kinds of problems that are worth investigating and govern the implementation of particular methods 
(Schwandt, 2001; Solomon, 2002). Methods, then, denote specific procedures, tools, or techniques utilized by an inquirer to generate justifiable knowledge (Schwandt, 2001). As such, the various arguments surrounding the notion of what constitutes "science" are all more or less based upon the adoption of one of two general doctrines: realism or relativism. At the extremes of each of these two doctrines, views are espoused which are in absolute opposition of the other. However, there are various components of each of the doctrines which in union create a sort metaphysical structure characterized by fractal cycles (Abbott, 2001). Thus, while some arguments stay firmly grounded in the extremist views of either realism or relativism, others maintain perspectives that blend the two doctrines together.

Metaphysical realism is an ontological doctrine that views the world as existing independently of consciousness and embraces the notion that there are universal truths to be found (Schwandt, 2001). Epistemologically, realism holds that universal truths exist and through experience we can come to understand the workings of the world (Hollis, 1994; Schwandt, 2001). Metaphysical realism can then be broken down into two opposing theories of human perception. Naïve realism holds that meaning exists in objects of study and humans as perceivers can directly, objectively and accurately decipher those meanings. That is, objects in the world appear just as they exist and humans perceive them as such (Schwandt, 2001). Accordingly, the warrant of a knowledge claim from a naïve realist's worldview is one of an objective ability to know things as they really exist (Schwandt, 2001).

In contrast, representative realism is the ontological belief that humans do not directly perceive external objects but rather the effects (i.e., images, ideas, impressions, 
etc.) that these objects have on them. Consequently, representative realism starts to integrate premises of subjectivity, whereby although there may be universal truths, our ability to know these truths is subjective in nature (Schwandt, 2001). The distinctions between naïve realism and representative realism epitomize the debate between subjectobject relationships (i.e., our ability as knowers and thinkers to know what we know and think what we think about objects of study).

The components of metaphysical realism are usually contrasted with metaphysical views of relativism. Ontological relativism indoctrinates the premise that reality is only that which can be determined by our language and/or conceptual schemes. In this regard, epistemological relativism separates the body and the mind and holds that phenomena is experienced only in appearance and situated in the cognition of the mind (Schwandt, 2001). Relativistic views often suggest that, although there may not be universal truths, there are many contextual truths about the world that we can come to "know" relative to some theoretical, conceptual, or cultural scheme. As such, relativistic beliefs are often associated with universal perspectivalism (i.e., all knowledge is interpreted and thus a reflection of individual perspectives) (Schwandt, 2001). At its extreme, relativism leads to radical skepticism (i.e., validated and universal knowledge is not possible) whereby knowledge claims can never be certain and always serve as interpretations of interpretations (Schwandt, 2001; Solomon, 2002).

Another ontological element to consider is the beliefs that one holds about the basic components of reality that account for what we can know and understand. One such ontological view is that of materialism which posits that the fundamental components of the world are physical or material subjects or objects (Solomon, 2002). In contrast, 
immaterialism denies components of reality as being physical or materialistic and acknowledges underlying intangible entities of reality (e.g., spirit and mind) (Solomon, 2002). Alternatively, essentialism is a worldview that in many ways unites the underlying premises between materialism and immaterialism. It is an ontological doctrine that holds that things or objects have essences or identifiable properties that characterize their true nature (Schwandt, 2001).

Ontological theories about the compositional features of the world are also linked to theories about how these features come together to shape the world and reality that we recognize. Many of these theories are then separated into epistemological and methodological beliefs about how we can accurately conceive the ways in which the fundamental components are organized in the world. The ontological notions of determinism and free will are at the forefront of these metaphysical considerations and refer to the opportunities for and constraints imposed upon entities' ability to act at random or by their own free will. Two frequently contrasted ontological views from this regard are theories related to stances of structure and action. These theories are then translated into the epistemological and methodological perspectives of individualism and holism.

Broadly conceived, determinism is thought of as a thesis that implies there is causal order in nature (Hollis, 1994). However, an inherent ambiguity exists pertaining to the strength and necessity of the causes. Thus, the engagement of deterministic premises spans a range of propositions. For example, at one extreme of determinism, Newtonian mechanics and physics posit there are absolute laws of nature that are universally and necessarily held in all places and times (Hollis, 1994). Conversely, a looser conception of 
determinism integrates facets of uncertainty, randomness and opportunities for freedom of will. Hence, a looser deterministic conception often contrasted with Newtonian views is Einstein's notions of a relativistic universe.

From a sociological standpoint, determinism is often linked to the conscientiousness of choice and action. One ontological approach encompasses theories that embrace the perspective of self-action which conceive of entities as acting under their own powers (Emirbayer, 1997). An extreme conception of self-action for human behavior is one that fundamentally explains action as being strongly determined by rational and conscious choice. The underlying assumptions pertaining to these theories predicate an atomistic viewpoint whereby people make their choices independent of others and their choices are guided by deep-seated, relatively enduring characteristics (e.g., personality, interests, etc.) (Emirbayer, 1997).

Some conceptions of self-action also maintain various externally deterministic premises, albeit more or less loosely conceived. For example, the idea of norm-coerced actions depicts individuals as self-subsistent, inner-driven individuals that pursue actions guided by fixed social ideals (Emirbayer, 1997). In this regard, action is conceived as less rationalized (i.e., calculated and conscientious) and a matter of conformance to idealistic socially-determined norms. The notion of free will comes into play in that individuals have a choice to conform or deviate from the idealistic choices for action.

Epistemologically, theories of atomistic self-action engender a "Bottom-Up" approach to thinking whereby individualistic methodologies are the appropriate approaches to inquiry (Hollis, 1994). Individualism maintains that self-driven actions of individuals aggregate together to form a social collectivity. Therefore, inquiries and 
explanations regarding social behavior focus on such features as the attitudes, actions, and decisions of individuals (Schwandt, 2001). From these premises, the methodological practice of reductionism ensues which entails the assumption that social "wholes" (i.e., collectivities) are reducible to parts that are construed as being capable of explaining the activities of individuals that comprise the wholes (Schwandt, 2001).

Contrastingly, a second variety of ontological approaches embody theories of structure which suggest the world is driven by forces that supercede individualistic attributes. In these approaches, self-subsistent entities (e.g., societies, structures, and socially deterministic classifications) are construed as exclusive and powerful sources driving action (Emirbayer, 1997). In addition to characterizing an ontological belief, structuralism is also a methodological approach for understanding the world which attempts to identify and describe underlying deterministic structures (Schwandt, 2001). Structuralist theories adopt the assumption that deterministic entities possess emergent (i.e., not readily observable) properties that are not reducible to discrete elements for the purposes of study and explanation. In other words, the driving forces of action must be conceptualized relative to the larger systems in which the entities belong (Emirbayer, 1997).

With these foundational arguments, structuralist thinking invokes a "Top-Down" conception of human action in which the behaviors of people are the deterministic result of underlying structures within a larger system (Hollis, 1994). As such, structuralism incurs methodological holism whereby explanation and understanding of social phenomena require the consideration of a macroscopic view of the larger systems. Holism is fundamentally opposed to the idea of explanatory reductionism because it 
assumes that the actions of individuals are a function of their relationship to a larger system. Thus, holism denies the ability to reduce explanation of the social whole to the parts of which it is comprised (Hollis, 1994; Schwandt, 2001).

An ontological alternative to the notions of self-determined action and structurally-determined action is the idea of inter-action. Under this view, entities do not generate their own action, but rather action is a function of causal interconnections (Emirbayer, 1997). Throughout an interaction between two entities, the entities themselves remained fixed and unchanging.

Epistemologically and methodologically this view of interaction has become a dominant approach to inquiry and knowledge acquisition through the implementation of "variable-centered" analyses. These types of analyses often employ various types of quantitative methods to test causal hypotheses using such analytical techniques as multiple regression, factor analysis, and event history approaches (Emirbayer, 1997). As Emirbayer (1997) highlighted, Andrew Abbott described variable-centered approaches as establishing an "imagery of fixed entities with variable attributes that 'interact, in causal or actual time, to create outcomes, themselves measurable as attributes of the fixed entities'” (p. 286).

All of the ontological beliefs described above fundamentally regard the governing components of the world as relatively enduring, static entities. The self-directed rationalactor theories, norm-based approaches, holistic structuralisms and variable-centered interactional views all espouse ontological, epistemological and methodological premises 
positing that the entities come first and the relationships among them are only of subsequent concern (Emirbayer, 1997). Emirbayer (1997) classified these ways of thinking into what he calls a substantialist perspective.

Fundamentally opposed to the ontological belief that the world is comprised of and is operationally constituted by static substances (i.e., things, beings, essences), Emirbayer (1997) and other scholars contend that the world is established by dynamic, unfolding relations and action is construed as a function of trans-action. Thus, a relational view of the world holds that substances cannot be understood as independent and are inseparable of the relations in which they are situated; in a sense, then, the world is construed as processes of ongoing negotiations. Emirbayer (1997) described:

Relational theorists reject the notion that one can posit discrete, pre-given units such as the individual or society as ultimate starting points of sociological analysis (as in the self-actional perspective). Individual persons, whether strategic or norm following, are inseparable from the transactional contexts within which they are embedded...By the same token, structures are empty abstractions apart from the several elements of which they are composed; societies themselves are nothing but pluralities of associated individuals (p. 287-288).

Furthermore, Emirbayer articulated, Variable-based analysis (as in the interactional perspective) is an equally unviable alternative; it, too, detaches elements (substances with variable attributes) from their spatiotemporal contexts, analyzing them apart from their relations with other elements within fields of mutual determination and flux (p. 288). 
In essence, a relational ontological view raises an intriguing alternative to the "Bottom-up” and “Top-down” approaches of individualism and holism. To convey this alternative approach, it is useful to draw upon Hollis' (1994) depiction of two divergent values of inquiry: explanation and understanding. Explanation predicates the idea that facets of nature are intelligible and allocate distinctions of determinable causal actions. The aim of explanation in this regard is often associated with conceptions of positivism (which will be covered in-depth later) and usually pertains to nomological accounts of action. Nomological explanations are characterized by an establishment of generality, universality, atemporality and causal explanation (Schwandt, 2001). A prominent view of what constitutes a nomological account of explanation is one that embraces a covering law model of explanation (i.e., aim of inquiry is to seek general causal or probabilistic laws for explaining action) (Schwandt, 2001). As such, Hollis’s (1994) characterization of explanation aligns well with realist ontological beliefs.

In contrast, Hollis (1994) referred to understanding as a need to understand the world from within rather than seek explanation from without. Thus, instead of seeking universal lawlike explanations for action, understanding necessitates an account of the meaning of action. The aim for understanding is generally derived from relativistic ontological views and aligns with interpretivistic (i.e, subjective accounts of action) and hermeneutic (i.e., the art, theory and philosophy of interpreting meaning) methods of inquiry (Hollis, 1994). The practice of understanding in this regard regularly incurs a historicist (i.e., narrative of the sequence of events preceding) account of action. In the context of trying to explain human behavior, Hollis's delineation of understanding holds 
that "actions derive their meaning from the shared ideas and rules of social life, and are performed by actors who mean something by them” (p. 17). Hence, the aim of understanding is to search for those meanings from within.

In contemplating the metaphysical bases of beliefs about the world and the way it works, several underlying themes emerge. First, regardless of the specific beliefs one holds to be true, the ultimate determination of what is fundamentally real is an understanding of some sort of properties that are relatively enduring in nature (e.g., objective understanding vs. subjective interpretation or substantialism vs. relationalism). A second derivable theme is the notion that reality is by some means unitary. That is, whether a person embraces “Top-down” holistic views, “Bottom-up” individualistic perspectives, or interpretive understandings from within, there is an intrinsic aspiration to know how the components of the world fit together to form a "whole" or "wholes" depending on your worldview (Solomon, 2002). Lastly, in constituting our knowledge claims we necessitate ways (albeit through various means of inquiry) to justify our beliefs and support our claims.

\section{Philosophies of Scientific Inquiry}

As was delineated earlier, much of the ambiguity and tension surrounding what constitutes "science" is derived from the adoption of opposing metaphysical views. Due to the complexity, and many times blending, of the various ontological, epistemological and methodological stances, it is difficult to provide a universally accepted definition of 
what constitutes “science.” However, a narrative overview of a few of the many philosophical perspectives on the nature of science can help clarify a number of the conflicting views.

The physical sciences have a tradition characterized by a relatively streamlined and rigid paradigm of scientific activities. That is, the conventional "scientific method" is the guiding structure behind most fields of inquiry and there is a strong tendency toward strict realist ontological and epistemological principles (although practicing scientists may not acknowledge or even be aware of the metaphysical underpinnings behind what they do). Consequently, many argue that in this way, the physical sciences are comprised of a single, linear, and cumulative collection of what they view as considerably objective facts. With a strict focus on empirical evidence, once a knowledge claim is deemed justified, the findings of a particular study are expected to matriculate into the evergrowing progression of scientific knowledge.

In contrast, the social sciences have multiple views regarding the metaphysical ontological and epistemological debates. As a result, the commensurability of knowledge and recognition of "facts" is an arduous challenge for the social sciences. In essence, as Alexander (1987) denoted, the number and extent of discourses reigning in the social sciences implies an "over-determination” of social facts by theory and an "underdetermination” of explanations by conclusive empirical evidence.

Moreover, an inability to effectively separate the subject from the object of study often leads to inconsistencies in empirical findings and an inconclusive determination of the source of the discrepancies. Consequently, rather than the single discourse predicated by the scientific method in the physical sciences, the social sciences invoke a multitude 
of discourses that span a continuum of strategies for "scientific" inquiry. At one extreme, the social sciences embrace a philosophy of naturalism (i.e., belief that human sciences should follow the same aim and methods as the physical sciences), and at the other extreme, the philosophies become blurred with those of the humanities (Denzin \& Lincoln, 2003).

In the most scrupulous sense, social science is characterized by an epistemological stance of strict empiricism. Commonly referred to as positivism (and sometimes 'positive science'), which was a term coined by August Comte, this stance holds that only the only legitimate knowledge claims are those established directly by experience (Hollis, 1994). In this regard, Comte sought to advance the distinction between theological, metaphysical and what he called "positive" knowledge claims. According to Schwandt (2001), Comte argued that the aspiration of science was “prediction accomplished by laws of succession” (p. 199).

In contemporary philosophies of science, positivism has come to be viewed as an umbrella term used to refer to people who base their theory on empirical observations for explanation (Hollis, 1994). Hollis articulated that positivists' science "goes with an empiricism about scientific knowledge, which rests on observations as the moment of truth when hypotheses are tested against facts of the world” (p. 42). In its most stringent sense, positivism can take on a pejorative connotation whereby a position of complete objectivity and naïve realism is adopted and is sometimes used to refer to someone who is myopically quantitatively focused.

A more general summary of the basic characteristics of a positivist paradigm include an aim of scientific explanation and prediction through as value-free of a lens as 
possible (Glesne, 2006). Positivists usually embrace an ontological view that there is one reality which can be quantified and measured. Epistemologically, positivists believe that reality consists of facts and with appropriate methods scientists can ascertain those facts (Denzin \& Lincoln, 2003). As such, positivists are usually characterized by a belief that, if an investigation follows the rigid methods set forth, and establishes a degree of methodological validity and reliability, the results can be considered objective and valuefree facts of the world.

\section{Perspectives on the Construction of Knowledge}

Through the lens of a positivistic paradigm, science has traditionally been portrayed as an individual practice of discovery whereby individuals through their own brilliance and luck of circumstance advance knowledge claims. The unified agreement on many of the metaphysical underpinnings guiding scientific practices lets scientists focus their attention on empirical questions, and scientific facts are deemed the outcomes of effective problem-solving (Alexander, 1987). Unlike the social sciences and humanities where classics (i.e., earlier works with a privileged status) serve an important role in theoretical development, the physical sciences employ exemplars (i.e., concrete examples of successful problem-solving) as guides. In this regard, the view of science as a cumulative body of facts and theory driven by empirical results deems classics as unnecessary because it is only necessary to integrate what is useful from past theory while the rest can be disregarded.

Nevertheless, in the 1960's and 1970's, the strict empiricism of the positivistic philosophies was heavily challenged by historians and philosophers of science (e.g., 
Kuhn, 1970; Merton, 1968). For example, Kuhn (1970) articulated the danger of the lack of recognition of classics in science texts:

Those texts have, for example, often seemed to imply that the content of science is uniquely exemplified by the observations, laws, and theories described in their pages. Almost as regularly, the same books have been read as saying that scientific methods are simply the ones illustrated by the manipulative techniques used in gathering textbook data...The result has been a concept of science with profound implications about its nature and development (p. 1)

Among the many contributions of Kuhn's seminal work, The Structure of Scientific Revolutions, was his coining of the term "paradigm.” Although there is some confusion surrounding an explicit meaning of the term, in part due to a lack of conceptual clarity on Kuhn's end, two distinct uses of the term emerged in his responses to the critics. In the first sense, a paradigm refers to a cognitive framework or shared solutions (i.e., exemplars) to substantive problems used by a community of scientists (Schwandt, 2001). The second connotation of the term pertained to a disciplinary matrix (i.e., commitments, beliefs, values, methods, outlooks, etc.) shared by scientists engaging in a particular discourse (Schwandt, 2001). While the first sense of the word, as noted earlier, does not readily lend itself to the social sciences (i.e., due to a lack of consensus on worldviews exemplars are not often recognized), the second sense of the word has often surfaced in deliberations between quantitative and qualitative research methodologies.

Hence, facing much criticism, and as a result of several seminal works on the philosophy of science, many of the extremist features of positivism have given way to views embodying postmodern conceptions. In general, postmodernism is an attitude 
toward the social world at the current stage of its historical development (Schwandt, 2001). Rather than the modernist views of humanism (i.e., autonomous individualism and deterministic structuralisms) and history as a linear process of progressive change, postmodernism calls for de-centered subjects, relational thinking and a view of history that is non-linear, cyclical and indeterminant (Lather, 1991). Schwandt (2001) expressed that postmodernism is characterized by its opposition to four central doctrines relative to conventional academic discourse: 1) the notion of a rational, autonomous subject—a self that has an essential human nature, 2) the notion of foundationalist epistemology, 3) the notion of reason as a universal, a priori capacity of individuals, and 4) the belief in social and moral progress through the rational application of social scientific theories to the arts and social institutions.

Consequently, a number of postpositivistic philosophies of science are now common among scientific research domains. Although there is much confusion surrounding the term postpositivism in the literature, broadly conceived it is the foil to the philosophies of science characterized by positivism. Some use "postpositivism" as an allencompassing term characterized by opposition to any of the ontological and epistemological views of positivism (Lather, 1991). Others use "postpositivism" solely to refer to a view that holds to the positivist views of one true reality and naïve realism, but stray from positivism epistemologically and methodologically. That is, this narrower view of postpositivism takes on a premise of representative realism (i.e., the ontological belief that humans do not directly perceive external objects but rather the effects these objects have on us). Thus, under this view postpositivists acknowledge that reality can never be fully apprehended, only approximated (Denzin \& Lincoln, 2003). 
Philosophical views that are closely tied to postpositivism (whether falling under it as an umbrella term or separate paradigms all together) are those of interpretivism and constructivism. Like postpositivism, there is ambiguity in the literature about whether they are actually two separate worldviews or characterize a single paradigm. For the purposes of this paper, the two will be described as falling under one philosophical view of constructivism.

A general conception of constructivism is characterized by an epistemological view of subjectivity. That is, constructivism holds that "human beings do not find or discover knowledge so much as construct or make it” (Scwhandt, 2001). Ontologically, constructivism espouses relativistic views of many truths characterized by local and specific constructed realities (Denzin \& Lincoln, 2003). The aim of constructivism is generally one that embraces Hollis' (1994) depiction of understanding. In other words, constructivists aim to understand the world from within rather than seek explanation from without.

As the discussion in this section delineates, in all reality, the social sciences (and in some respects the physical sciences too) have not achieved consensus in determining a single philosophy of science. In essence, there are many philosophies and beliefs that have gained a relative acceptance (or at least recognition) as possible approaches to scientific inquiry. Fundamentally, as Kuhn's (1970) notion of paradigm explicates, the scientific endeavors academics pursue and the means by which they pursue them are inevitably shaped by historical, political and social forces. That is, the ontological, epistemological and methodological worldviews that one holds to be true as well as the topics selected for investigation are likely products of historically-informed interactions 
(both directly and indirectly) with other people and subject to deterministic forces of social and political norms.

Fundamentally, perspectives on the construction of knowledge claims fall into one of two categories: 1) a positivistic view in which if knowledge claims are obtained through straight-forward methods whereby a relative degree of validity and reliability are established, they are considered to be value-free facts about the world, and 2) a constructivist view which holds that knowledge claims are inherently subjective and influenced by historical, social, and political factors. Similarly, a number of theories exist which attempt to explain how knowledge claims are accumulated and assimilated into general bodies of knowledge. These views are also varied in terms of the ideas they suggest, but tend to embrace either positivistic or constructivist tendencies. As such, the upcoming sections provide an overview of some of the more commonly cited models on scientific growth and progression.

\section{Popper's Process of Conjecture and Refutation}

A renowned philosopher of science, Karl Popper, advanced the principle of falsification to distinguish scientific endeavors from nonscientific ventures. As a fallibilist, Popper argued that scientific claims, at least in principle, are capable of being found incorrect while non-scientific claims are not (Schwandt, 2001). Popper contended that scientific knowledge thus advances through a process of "conjecture and refutation" whereby scientific claims are never actually provable or conclusively justifiable.

Fundamentally, Popper's process of science unfolded as such: 1) an inquirer identifies a problem to study, 2) inquirer puts forth propositions that serve as initial 
conjectures or hypotheses of explanation, and 3) research and analysis then either confirm or reject the conjectures (Calhoun et al., 2002). As a philosophy of how scientific knowledge grows, Popper's principle of falsification has been criticized as an untenable explanation. Nevertheless, many his of contentions relative to methodological proceedings, are still invoked in research practices today (e.g., disconfirming hypotheses rather than verifying hypotheses in quantitative analyses and using negative case analysis in qualitative studies) (Schwandt, 2001).

One of the criticisms of Popper's model of conjecture and refutation as an explanation for scientific growth is that "facts and cases are not strong in and of themselves, but only relative to the strengths or weaknesses of competing accounts" (Fuchs \& Ward, 1994, p. 487). In other words, you must have an alternative hypothesis for comparison purposes to "falsify" an old one (Fuchs \& Ward, 1994, p. 487). Likewise, as Quine's notions of webs of belief maintained, all statements (including the logical connections of theoretical statements) derive their meaning from how they are embedded in webs of other terms. As such, Quine argued this web provides defenses for the nonconforming observations and suggests the notion that we can choose which parts of a theory are rejected by evidence (Hollis, 1994). Moreover, as Fuchs (1993) articulated Popper's model espouses a view of scientific findings as being cumulative in nature and does not account for historically, socially and politically informed conduits for knowledge change and growth. 


\section{Kuhn's Paradigmatic Shifts}

The contentions elucidated in Kuhn's $(1962,1970)$ The Structure of Scientific Revolutions is often attributed as a large factor in the discrediting of Popper's model of scientific growth (Fuchs, 1993). As was described in an earlier section, Kuhn's notion of paradigms incited a strong argument against the belief that scientifically produced knowledge is objective, neutral, and value-free. Fundamentally, as Glesne (2006) described, "Kuhn demonstrated how science was often an ideological battleground where ideas and explanations competed, and those that "won" were often those of the scientists with the most power (economically, politically, socially, etc.)” (p.7). As such, the thrust of Kuhn's model for the growth and change of scientific knowledge was his distinction between Normal and Revolutionary science.

In a brief overview of his arguments, Kuhn's model suggested that scientific growth and development is derived from the establishment of a paradigm (or accepted model) which sets forth the guidelines and expectations for research to ensue. With a paradigm in place, a period of Normal science follows in which scientists explore various applications of the paradigm and the results produce a relatively coherent body of knowledge. Kuhn (1970) articulated, "Normal science, the activity in which most scientists inevitably spend almost all their time, is predicated on the assumption that the scientific community knows what the world is like” (p. 5).

From time-to-time, empirical evidence arises that no longer conforms to the theory ascribed to the paradigm; thus invoking a state of "crisis" in the field. The formulation of a new paradigm that helps fill the voids of the old paradigm is proposed and a battle of paradigms ensues. At the culmination of the battle, if the new paradigm 
wins out, a transformative revolution characterized by a shifting of paradigms takes place. Consequently, over time the new paradigm enjoys a period of Normal science with the potential to be challenged by a later period of crisis and revolution.

What was particularly useful about Kuhn's work was how his model was able to capture (albeit modestly) how two different paradigms vie for dominance and legitimacy in the field. Unlike Popper's principle of falsification, Kuhn's model recognized that findings from various studies and approaches could be rendered "incommensurable," eschewed the idea of objective and cumulative scientific progress, and introduced propositions about how history, politics and social relationships play into the research process. Nevertheless, although many of Kuhn's propositions have become widely recognized and accepted, several scholars have revealed the limitations of his views.

One of the main criticisms of Kuhn's model is the very linearity of his framework. Although he accounted for distension and incommensurability, his portrayal of the "paradigm wars" predicates that one dominant paradigm wins out in the end. As Collins' (2000) analysis of the survival of "high visibility gravitational radiation” (HighVGR) demonstrated, however, even in the physical sciences a plurality of contradictory paradigms can simultaneously exist within a single field. Fundamentally, Collins' study showed how, in spite of empirically falsified evidence and a general fieldwide informal rejection of the HighVGR paradigm, research embracing this paradigm continued to exist with formal support (i.e., peer-reviewed publications and funding) for nearly a quarter of a century. Likewise, Kuhn's model does not account for the subsistence of the multiple philosophical paradigms widely implemented in the social sciences (Abbott, 2001). 


\section{Fuch's Sociological Theory of Scientific Change}

In a similar manner, Fuchs (1993) denoted that Kuhn’s model does not account for the many possible reactions to anomalies that arise in scientific activities (i.e., one can ignore them, explain them away, make minor modifications in a trivial part of the theory, etc.). Furthermore, Fuchs (1993) argued that Kuhn's model only expects two basic types of scientific activity: normal and revolutionary. However, Fuchs (1993) expressed, Most scientific change appears to be nonrevolutionary. Holton (1988), in his study of relativity theory, has suggested that revolutions are often returns to classical ideals of simplicity and uniformity...It seems that the major failure underlying these various problems with Kuhn's theory is the failure to allow for more variation in scientific practice. The normal/revolutionary dichotomy is not complex enough to account for the many ways in which science may change (p. 934)

Consequently, Fuchs (1993) proposed what he called "the sociological theory of scientific change.” Fuch's theory views scientific specialties (i.e., a group of practitioners with similar training, attending the same workshops, reading and citing the same bodies of literature, etc.) as material resources and inherent social structures that shape how scientists perform their work.

Fuch's theory called upon the literature in organizational theory to conceptualize scientific change in specialties, demarcating two factors relating to the permissibility of innovation: the structure of the group and formality of group organization. According to Fuchs, the more cohesive and homogenous group, the greater the pressure to conform to its norm-based rules and standards. In contrast, loosely coupled and heterogeneous 
specialties allow less rigid styles for cognitive pursuits. Likewise, when the work is certain, routine and predictable, the group’s organization will resemble a Weberian bureaucracy. Thus, the academic specialty characterized in this regard emphasizes conceptual orderliness and procedural correctness. Conversely, if the tasks are uncertain and nonroutine, the social structure of the academic specialty will be less formal, more flexible and decentralized—and hence, more tolerant of innovation and nonconformance. As such, Fuchs (1993) suggested, Strong and closely coupled organizations produce science; loosely coupled and textual organizations produce hermeneutics. Weak textual disciplines are more discursive and metaphysical than factual and objective. Their intellectual culture is comparatively cosmopolitan and relativist...The sheer plurality of worldviews and ideologies reveals the contingency of all world views. For none of the many approaches can, without strong and persistent opposition, claim special or privileged status (p. 946).

Under these premises, Fuchs contended that innovative research fronts and routinized paradigmatic approaches seem to be much more characteristic of the physical science than of the social sciences or humanities. In essence, he argued against the dominant philosophical argument between the social and physical sciences is that there deep-seated ontological and epistemological differences between the study of natural and social entities. Fuchs (1993) suggested that the difference is not in the objects of study, but rather the structures of the groups and organizations doing the studying.

In a later article, Fuchs and Ward (1994) elaborated on Kuhn's shortcomings regarding the fact that not all scientific change is revolutionary. They delineated, 
minimalist realism is broadly conceived of as the philosophy of most practicing scientists who engage in what Kuhn (1970) referred to as activities of normal science. Scientists in this regard either have no interest in or find relevance for the metaphysical debates on ontological and epistemological views (Fuchs \& Ward, 1994). As such science conducted in this matter turns what Fuchs and Ward referred to as "radical DECONSTRUCTION" into moderate deconstruction. Fuchs and Ward (1994) expressed, In deconstruction, some group or organization tries to construct a certain state in the world, while their opponents or competitors intend to construct a different state, which implies, at the same time, deconstructing their opponents' construction. Both forms of deconstruction weaken the authority of statements, but deconstruction is much less radical than DECONSTRUCTION. While DECONSTRUCTION wants to change the game or play a different game altogether, deconstruction wants to play the game and challenges only certain moves of certain competitors within it (p. 484).

Fundamentally, Fuchs and Ward's conception of DECONSTRUCTION and deconstruction differ in degree, not in principle. In general, almost all scientists engage in deconstruction as it is part of the process of conjectures and refutations, however, under certain circumstances (as in the example of Kuhnian revolutions) scientists will partake in activities of DECONSTRUCTION (Fuchs \& Ward, 1994).

\section{Crane's Invisible Colleges}

From a different perspective, Crane (1988) advanced the notion of invisible colleges as a means of describing and explaining scientific knowledge growth. 
Challenging the fact that many models of the growth of scientific knowledge describe the cognitive aspects of growth, Crane sought to explore the role of scientific communities in the research process. The first step in Crane's model was to establish that the scientific growth of knowledge was indeed a function of social interaction. To do so, she employed a model of scientific growth as a social diffusion process. Crane (1988) articulated,

If scientific growth represents the accretion of many small innovations, and if, in producing these innovations, authors are indeed building upon each other's work (as analyses of their citations to each other's publications suggest), then it would appear that such authors are adopting some of each other’s innovations (p.22). Under these premises, Crane suggested scientific knowledge can be viewed as a process of social contagion.

As a result of this part of her analysis, Crane found evidence to suggest that in stage one of a paradigm, interesting discoveries attract the attention of new scientists. In stage two, a few highly productive scientists set priorities for research and often recruit and train students (who then become future collaborators) which ultimately stimulates an exponential growth of publications in the area. Finally as anomalies emerge, fewer new scientists are attracted to the paradigm and old members are likely to move on to new areas characterizing a lull or burn-out in interest.

The second part of Crane's model considered the social organization of research areas. Crane's focus in this section was on identifying the various types of social relationships that take place within a research area and how those relationships impact scientific knowledge growth. As such, Crane suggested that some of the most important indicators of social organization in research areas include: informal discussions of 
research, published collaborations, relationships with teachers, and the influence of colleagues upon the selection of research problems and techniques” (p. 41). In analysis of some of these social networks, Crane found evidence for the presence of what is often referred to as "scientific stars.” That is, members of research area networks were not so much linked directly to each other but were linked indirectly through a few highly influential members of the research area.

The third facet of Crane's model then drew a direct connection between the social organization of research areas and the diffusion of ideas through it. Fundamentally, her analysis indicated that social factors within a research area affect the dissemination of the ideas through the network. In addition, Crane found evidence that social factors were also correlated with the likelihood that ideas were again utilized in later publications.

She interpreted the latter result as being related to either the effect of the quality of the ideas or as the effect of a social influence process that increases the visibility of previously utilized work. Crane contended:

A high level of utilization reflects conformity to norms set by the invisible college in the area. Innovations that were more frequently utilized or cited by members of these research areas were those that were utilized or cited by the most productive members of the area (p. 83).

A fourth premise of Crane's invisible colleges investigated the notion that paradigms may not shift but rather split into different communities or schools of thought. Crane's model suggested that confrontation may not ensue between paradigms because supporters of different paradigms devote their attention to different research areas or 
those that do pursue the same area avoid confrontation with each other by minimizing points of contact (i.e., citing those who follow their own school of thought). In addition, Crane suggested, that attention to new research areas might not necessarily be a result of anomalies as much as socially fashionable trends.

Finally, Crane's model also raised the issue of how closed or open specific disciplines are to the external influence of other disciplines. Crane articulated, If research areas were completely closed to external influences, scientists would be divided into small groups, sharing the same interests, speaking only to each other, and reading and citing only each other's work. If this were the case, science would consist of hundreds of disparate groups, none of which would have any communication with or relevance for each other (p. 100).

Her findings suggested that exchange of different ideas between members of distinctive research areas is important in terms of generating new lines of inquiry and in producing some integration of findings from diverse areas. Likewise, Crane’s analysis indicated that some degree of closure is necessary for a college in order for knowledge bases to accumulate and grow.

\section{Abbott's The Chaos of Disciplines}

Turning more specifically to the structural barriers and opportunities in regard to theoretical development, Abbott (2001) proposed a model of scientific growth for the field of sociology (although noted that his propositions has theoretical implications for other social/cultural organizations as well). The first feature of Abbott's model pertained to the interstitial nature of the social sciences which he noted "stands uneasily" between 
the natural sciences and the humanities; a middle ground between "the mode of facts and the mode of values” (p. 7). Abbott described sociology as a field of permeable theoretical boundaries due to its implicit inability to exclude ideas from the field once they are introduced.

The second thrust of Abbott's model was to refute the linearity of theoretical develop and instead adopt a model of fractal distinctions. Using the binary of the classic “qualitative” and "quantitative” traditions to explicate his thinking, Abbott argued that sociology is characterized by split dichotomies that then split and split again. In his depiction, Abbott illustrated how out of the topic of methods a split created a dichotomy of a quantitative/qualitative divide. Each side of the dichotomy then splits again into a new lineage of a quantitative/qualitative divide under each of the earlier dichotomized groupings (i.e., the first line of quantitative methods splits into a second dichotomy whereby within quantitative methods qualitative methods also emerge). As such, Abbott (2002) expressed,

A fractal distinction thus produces both change and stability. Any given group is always splitting up over some fractal distinction. But dominance by one pole of the distinction requires that pole to carry on the analytic work of the other, so the endless subdivision that we label by the word differentiation does not seem plausible. There results a continuous bending of terminologies that breaks down the original metaphors that produced the dominance (p.21).

Abbott's third proposition then entails what he termed "fractal cycles.” As Moody (2004) described, Abbott's model conveys a structure whereby "Pushed by competition for status, proponents of one set of ideas attempt to vanquish another, only to find that 
they need to reinvent those same ideas later” (p. 217). In essence, Abbott's fractal cycles described a constant reshuffling and revisiting of ideas creating an ever-growing body, though not linear in nature. Furthermore, as the methods we employ fundamentally shape our findings, Abbott articulated an advantage of breaking down the extremist affinities of the quintessential methodological quantitative/qualitative divide claiming:

Breaking these affinities is the most powerful mechanism for knowledge change in the social sciences. Interesting new social science can always be produced by trying a combination of the hitherto unknown (p. 29).

\section{Research on the Sociology of Science}

During the last half of the century, historians and sociologists alike, have noticed an evolutionary change in regard to scientific practice and communication—a significant rise in terms of the collaborative efforts on the part of researchers. Research examining scientific collaboration conducted in the realm of the physical sciences has revealed a shift from the practice of scientists and theoreticians working in relative isolation to the development of research teams. Price (1986) demonstrated that in many cases, collaboration in the physical sciences now appears to be the norm. Likewise, studies on the social sciences have also shown that single authorship of research studies have decreased, while coauthorship has increased; not only in terms of the number of studies that entail multiple authors but also that the number of authors per article (Endersby, 1996). Historians and researchers falling into the humanities classification, on the other hand, tend to embrace sole authorship over collaboration (Ede \& Lunsford, 1990). 
The increases in collaboration rates can be attributed to a number of factors. For example, improvements in technology (e.g., email, travel, etc.) has rendered collaboration more convenient as well as broken down many of the previous geographic barriers. Likewise, Endersby (1996) presented several incentives for researchers to collaborate: 1) as scientific research becomes more complex, utilizing the expertise and talents of others can be beneficial, 2) active participation of students and junior colleagues can provide a valuable learning experience, 3) collaboration can lead to increased efficiency of output, and 4) the quality of research can be improved by the opportunities and constraints required in collaborative efforts. Whatever the reasons for the increases in collaboration, many scholars have articulated that the collaboration patterns between scientists has widespread implications for the diffusion of information and opportunities for scholars to influence each others’ work (e.g., Friedkin, 1998, Kuhn, 1970; Moody, 2004).

Nonetheless, as Crane (1988) expressed, "growth in gross numbers of publications is not a good indicator of the growth of knowledge on the grounds that only a few publications are heavily utilized in later scientific work" (p. 19). Thus, rather than taking on individual researchers or research papers as the unit of analysis, many scholars have stressed the need to examine how kinds of social interaction and organization affect the growth and changes in knowledge patterns specifically (e.g., Ennis, 1992; Swidler \& Arditi, 1994). Consequently, this avenue has been explored by a number of researchers investigating such topics as the effects of the informal and formal controls on the plurality of paradigmatic subsistence (Collins, 1998), scholars’ consensus and journal rejection rates (Hargens, 1988), and the implications of organizational features (i.e., task uncertainty, level of integration, authoritative influence, etc.) of scientific groups (Fuchs, 
1992; Fuchs \& Ward, 1994). Likewise, studies on citation patterns (e.g., Mizruchi \& Fein, 1999), joint-membership (Daipha, 2001; Cappell \& Guterbock, 1992), and shared specialties (Ennis, 1992) have produced interesting implications regarding the underlying social structures and knowledge growth.

More recently, researchers have explored direct linkage patterns between social interaction and the structure of ideas spaces (e.g., Crane, 1988; Friedkin, 1998; Martin, 2002; Moody, 2004). For example, citation analyses have generated detailed representations of some of the interrelations within and between specialties in various sciences (Crane, 1988; Small \& Crane, 1979). Indeed, Crane (1988) implemented such an approach by viewing scientific knowledge and growth as a process of social diffusion.

Crane (1988) and other scholars (e.g., Kuhn, 1970) have argued that the topics a researcher pursues and the paradigmatic approaches that guide his or her investigations are strongly influenced by those with whom the individual interacts. As discussed earlier, Crane's (1998) model explored how various facets of the networks of "invisible colleges" can impact the diffusion of information, citation patterns, and forces of attraction guiding scientists to certain areas of research. Likewise, Martin (2002) demonstrated that belief consensus in small networks is associated with the authority structure of a group's social network, and Friedkin (1988) suggested that consensus across different groups of scientists is related to overlapping cohesive groups of a larger social network. Furthermore, support for various implications of how the networks of collaborative efforts affect the overall knowledge spread and growth has been evinced in several ethnographic works on scientific disciplines (Collins, 1998; Owen-Smith, 2001). 
Given these arguments, a concrete mapping of the social structure of a scientific discipline is an ideal means to explore the opportunities scholars have to be influenced (as well as the opportunity to influence others) based upon their connectivity to all of the other scientists and location within the social structure of the discipline. As such, social network theory and analysis can serve as a valuable tool in studying various factors associated with the sociology of scientific generation in any academic field. Moreover, social network analysis provides the opportunity to extend the study of social influence in scientific activities beyond small groups of specialty areas and teams of scholars.

In fact, Moody (2004) used a network approach to explore the underlying social structure of an entire discipline. Examining the structure of the collaboration network over time, Moody investigated several claims from the models discussed earlier (i.e., star networks, fragmentation, and fractal walks/permeable boundaries) relative to the discipline of sociology. In addition to revealing some intriguing implications for the discipline of sociology as a whole, Moody’s (2004) approach also elucidated some of the advantages for using a network approach to studying entire disciplines.

As the overview provided in Chapter 1 denoted, social network methods provide compelling and useful techniques for capturing the social relations between actors embedded within a system of other actors. By providing tools for overturning the static, undersocialized and oversocialized limitations of the more conventional research methods, social network analysis is particularly useful for studying diffusion and social contagion patterns. Thus, the application of such techniques to the study of scientific knowledge growth and the underlying social structures of disciplines can be extremely advantageous. 


\section{A Social Network Perspective}

In some of the social sciences’ fields of study such as psychology and economics, human behavior tends to ultimately rest on individualistic assumptions (i.e., atomistic rational-actor models). Research investigations in the field of sociology, on the other hand, are often concerned with determining enduring patterns in the relationships among elementary parts of social systems which are thought to constrain individuals' behaviors (i.e., structural holisms) (Wellman \& Berkowitz, 1988). In addition, approaches, at times, blend each of these perspectives together especially when moving from descriptions of the observations to explanation or understanding of the observations.

Nonetheless, in any of these cases, rather than working from a holistic social structure, most researchers take the approach of working toward a larger structure through aggregative analyses (Wellman \& Berkowitz, 1988). That is, both conventional quantitative approaches (with the implementation of inferential statistics and probability models) and qualitative approaches (with the adoption of constructivist premises) have a tendency to "base their explanatory schema on the putative norms and values of idealized social actors” (Wellman \& Berkowitz, 1988, p. 3). As such, according to Wellman \& Berkowitz (1988), social scientists following these strategies can "at best, only infer the presence of social structure when they discover aggregates of individuals thinking and behaving in similar ways” (p. 3).

Both atomistic individualism and structural holisms tend to dictate thinking into terms of categories or groups of social actors who share similar attributes. While, depending on the research questions of interest, these approaches have their uses, analytically the outcomes derived are founded on assumptions that particular attributes 
are the driving forces of human behavior, humans act in isolation of one another, and actions are based upon independent events (i.e., not historically informed) (Wellman \& Berkowitz, 1988). Thus, as Wellman (1998) articulated, “although most mainstream sociologists profess to be studying social structure through attributional analyses, their inherent 'methodological individualism' leads them to neglect social structure among individuals” (p. 31).

The limitations of these approaches become all-the-more apparent when contrasted with the alternative network perspective. Rather than working toward an indirect and abstract understanding of social structure using categorized attribute variables, network analysts focus on studying social structures directly and concretely through observations of relations between individuals in a social system (Wellman \& Berkowitz, 1988). Wellman (1988) highlighted five characteristics of network analysis that set it apart from the traditional mainstream approaches:

1. Behavior is interpreted in terms of structural constraints on activity rather than in terms of inner forces within units (e.g., "socialization to norms”) that impel behavior in a voluntaristic, sometimes teleological, push toward a desired goal.

2. Analyses focus on the relations between units, instead of trying to sort units into categories defined by the inner attributes (or essences) of these units.

3. A central consideration is how the patterned relationships among multiple alters jointly affect network members' behavior. Hence, it is not assumed that network members engage only in multiple duets with separate alters. 
4. Structure is treated as a network of networks that may or may not be partitioned into discrete groups. It is not assumed a priori that tightly bounded groups are, intrinsically, the building blocks of the structure.

5. Analytic methods deal directly with the patterned, relational nature of social structure in order to supplement—and sometimes supplant—mainstream statistical methods that demand independent units of analysis (p. 20).

The implications of Wellman’s first characteristic are relatively clear based upon the earlier discussion. Wellman’s second premise aligns with Emirbayer’s (1997) deliberations on relational perspectives versus substantialist perspectives whereby selfacting and inter-actional approaches typify the conventional methods while network analyses embrace a transactional approach. In other words, rather than using substances or essences and their attributes as the starting points of analysis, network approaches focus first on the relationships between them. Thus, while mainstream approaches tend to collect data on attributes such as the relative strength of attitudes, perceptions and characteristics of units, network approaches concentrate on relational data such as the relative presence, strength and intensity of ties between units (Scott, 2001).

The third characteristic highlighted by Wellman refers to another approach commonly implemented in sociological studies: dyadic interactional relationships between units. While this approach does stray from methodological individualism by looking at factors affecting the initiation and continuation of ties as well as the types of resources each dyad exchanges with the other, it still incurs a form of "reductionist aggregation” (Wellman, 1988, p. 35). That is, approaches focusing only on dyadic exchanges still disregard the larger structural system within which the dyad is located. As 
such, these approaches adopt the assumption that isolated dyads can be representative of all other dyads in a system without regard to the nature of other ties in the network or to how all of the other ties fit together.

Consequently, this idea leads into Wellman's fourth characteristic which holds that the world is composed of networks not groups. Rather than conceiving of groups as collectivities of autonomous individual actors, network analysis precludes that the linkages among individual actors can constitute more or less bounded groups (Wasserman \& Faust, 1994). Wellman (1988) contended that the categorical groupings (e.g., neighborhoods or communities) of traditional sociological methods tend to oversimplify the true complexity of social structures. Thus, he articulated, "By starting with networks rather than groups, analysts are able to study both ties that do not form discrete groups and networks that are, in fact, sufficiently bounded and densely knit to be termed 'groups'” (p. 37). As such, network analysis allows the researcher "to describe linkages among the members of social systems and to manipulate these representations in order to probe the underlying 'deep structures' connecting and cleaving social systems” (Wellman \& Berkowitz, 1988, p. 23).

Broadly conceived, in a network approach, a group is a collection of actors upon which ties are to be measured (Wasserman \& Faust, 1994). Since, from a network perspective, any group is a more or less bounded network of actors, a single network can also be a host to more or less bounded groupings of actors within its specified boundaries. Moreover, these subgroups may have overlapping layers in that single actors may be 
connected to multiple subgroups at the same time. Therefore, researchers implementing a network perspective should be able to justify the group boundaries they set through theoretical, conceptual and/or empirical reasoning (Wasserman \& Faust, 1994).

Finally, Wellman’s fifth feature highlights the distinctiveness of a network perspective's ability to directly measure relational patterns as well as evaluate across multiple levels of analysis. According to Berkowitz (1982) network analysts "accept the view of science as fundamentally 'pattern recognizing': that its object is to create models which act as analogs for complex physical, natural or social phenomena which can then be observed in the 'real world”” (p. 7). In this regard, a network model serves as a simplified representation of some facet of "reality" that is reduced both in terms of scale and complexity (Berkowitz, 1982, p. 7). More specifically, a "social network” refers to a theoretically, conceptually, and/or empirically bounded set of actors and the ties among them. An analysis of the network thus aims at modeling the relationships among the actors to depict a concrete structure which can then be studied to investigate the impact of the structure on the functioning of the group and/or the influence of this structure on the individuals within the group (Wasserman \& Faust, 1994).

According to Wasserman and Faust (1994), network analysis amalgamates the process of model building by providing formal definitions of concrete relational concepts which can then be used to evaluate and test theories about relational processes or structures. The fundamental difference between traditional methods of analysis and network methods is the unit of analysis to which researchers devote their attention. Rather than focusing on individual actors and their attributes, network analysts concentrate on a specific more or less bounded set of actors and the linkages among 
them. Therefore, in network studies the "theoretical concepts are relational, pertinent data are relational, and critical tests use distributions of relational properties" (Wasserman \& Faust, 1994, p. 6). As such, network analytic measurements and theories can then be extended to serve as explanatory factors or variables in understanding individuals' and groups’ behaviors (Wasserman \& Faust, 1994).

One significant advantage a network approach imparts is a formalized vocabulary that has developed to represent relational definitions, concepts, and structural properties in precise and consistent ways (Wasserman \& Faust, 1994). Unlike the categorical approach used in more traditional methods which often entails, as Homans (1967) articulated, ambiguous and imprecise "nonoperating” and "orienting statements," a network approach uses unitary, formalized language and procedures to directly define concepts (Wasserman \& Faust, Berkowitz, 1982; Wellman \& Berkowitz, 1988). For example, using conventional methods, the phrases "social group" and "social role" involve contextual clarifications in order to be operationalized and measured.

In contrast, using a network approach, these concepts are formally, concretely, and consistently defined and therefore can be precisely measured and quantified among the actors in a network. In essence, as Wasserman and Faust (1994) contended, "The methods of network analysis provide explicit formal statements and measures of social structural properties that might otherwise be defined only in metaphorical terms” (p. 17). Hence, social network analysis provides a formalized and relatively concrete means of thinking and theorizing about the social connectedness of entities in the world (Wasserman \& Faust, 1994). 
As a network approach deals with conceptual and theoretical problems and questions that are very different from the conventional methods, traditional statistical and data analytic procedures (e.g., multiple regression, t-tests, canonical correlations, structural equation models, etc.) are generally not directly applicable for testing network data and theories (although they may implemented if structural elements discovered are later turned into structural variables). Thus, network analysts have developed an entire body of methods specifically designed for investigating relational concepts and properties. In particular, the precision and consistency of the vocabulary provided by a network approach allows for the integration of formal mathematical models as a basis for empirical testing and theoretical development. As such, three major mathematical foundations for network studies include graph theory, statistical and probability theory (used to study relational properties such as reciprocity and transitivity), and algebraic models (Wasserman \& Faust, 1994).

In addition, conceiving of the world as being comprised of more or less bounded networks rather than rigid categorical groups inculcates some interesting analytical implications. Conventional categorical approaches compel researchers to determine a priori specific levels (i.e., individual, subgroups, or entire collectivity) and units of analysis (usually attributes of entities) upon which to focus their observations. That is, a researcher must determine prior to empirical investigation whether they are going to investigate the properties or attributes of individuals in a population, positional properties of groups within a population, or a population in and of itself.

In contrast, social network analysis fundamentally predicates a unitary nature of social processes and thus places great emphasis on the mutual interaction and 
interdependence of systems, subsystems and the elements that comprise them (Berkowitz, 1982). By using relationships between entities as the primary unit of analysis, a network perspective allows a simultaneous investigation of multiple levels within a single study (Wasserman \& Faust, 1994). Therefore, using a network approach, a researcher can investigate how actors are located or “embedded” in the overall network as well as holistically assess the patterns of relationships among all of the actors.

Under these premises, network analysis posits a number of substantive opportunities for researchers to study the social structures of scientific disciplines. Of specific interest is the notion that relational ties between actors in a structure can be viewed as channels for the transfer or "flow" of resources (i.e., information, ideas, materials, etc.) throughout a system of actors. As Wellman and Berkowitz (1988) expressed,

A basic strength of the whole network approach is that it permits simultaneous views of the social system as a whole and of the parts that make up the system. Analysts are therefore able to trace lateral and vertical flows of information, identify sources and targets, and detect structural constraints operating on flows of resources (p. 26).

\section{A Social Network Perspective on the Social Construction of Knowledge} Moody (2004) used an innovative approach that tied theoretical antecedents and consequences in the sociology of scientific knowledge to a social network perspective. In doing so, Moody suggested that there are three competing models of scientific collaboration patterns that can be related to the social structure of a discipline. These 
three models included: 1) the scientific star model, 2) the small-world model, and a 3) model of structural cohesion. Each of these models is described in further depth below.

\section{$\underline{\text { Scientific Star Model }}$}

One common theme that has emerged from critical reflections on scientific disciplines is the notion that scientific production and idea diffusion depend heavily on a few "scientific stars” or prolific researchers of high status. Scientific stars are those researchers within a discipline who may tend to attract a disproportionate level of research funding, citations, students and aspiring collaborators because of their prestige and status (Moreno, 1968). Moody (2004) articulated, “Although most scientists labor in obscurity, a small number of scientists receive disproportionate recognition. This has been clearly demonstrated for indicators such as citations, number of publications, or grants” (p. 216).

In addition, Crane (1988) contended that the scientific stars are likely to play a large role in terms of determining what works get cited and the ideas that attract recognition in the discipline. For instance, people trying to make their way in the field often seek to work with the high-profile researchers and are thus likely to adopt the dominant ideas that have been developed by the scientific stars (Crane, 1988). Likewise, the high status individuals are also likely to be the very same individuals who serve on the editorial boards and as reviewers for peer-reviewed journals which ultimately suggests that these individuals can have great influence on what work gets published. Thus, in essence, a central location or "star" position in the collaboration network suggests that a researcher has the potential to rapidly diffuse ideas throughout the 
discipline, while the other researchers in the field often have to depend on the stars in order for their ideas to spread.

It is important to point out that although the use of the word "star" might seem to infer that the work of those located in a central position is the most innovative and quality work being produced in the field, this is not necessarily the case. While one reason a person has become a recognized "star" in the field may indeed be a result of the ideas he or she has published, the scientific star model simply refers to their structural position in the network and not the actual quality of their work. Specifically, a "star" in the scientific star model is one who has the potential to serve as a gatekeeper for the transfer of ideas.

In other words, while the individual is tied to a number of people, the people who they are connected to are not highly connected to each other. Hence, in theory, if the network is characterized by a few "scientific stars," the highly-connected stars have the potential to serve as very influential people within the discipline. Figure 1.1 provides a simple graphical depiction of what a network characterized by scientific stars might look.

\section{Disciplinary Small-World Model}

Many studies have noted that fields within the social sciences in general do not have an overarching theory or set of theories but rather possess theoretically fractured disciplines comprised of a large number of disconnected research specialties (Moody, 2004). Through a diffusion process, it may be assumed that new ideas will be rapidly developed and explored by a core of individuals who often interact either directly (through collaboration) or indirectly (via reading and citing others' work) with each 
other. As ideas get further developed and acceptance or consensus about an idea grows, it is possible that a core group of research specialists working within that idea space will become a highly clustered cohesive group of individuals.

Those scientists in opposition or disagreement about an idea will either be forced to fight a single-person battle against a collective group, form their own highly clustered and structurally cohesive group of individuals over time, or avoid the topic in general (Abbott, 2001). In some cases, those disinterested in pursuing the topic in the paradigmatic way deemed acceptable by the core group will simply focus their research elsewhere (Crane, 1988). Consequently, as Moody (2004) cited from Stinchcombe (2001), "The wide variety of substantive subject matter in disintegrated disciplines, and the strong boundaries around substantive specialties, means that people cannot get interested in each other's work” (p. 215).

Theoretical fragmentation is likely indicative of a highly clustered social structure of idea spaces where people outside the clusters are unable to or choose not to closely follow each other's work. If a discipline is theoretically fragmented and/or people are unable to closely follow each other's work, then rather than collaborating with people of diverse backgrounds, opinions, and beliefs, researchers are more apt to collaborate with fellow specialists in their topical areas (Moody, 2004). Consequently, the structure of a discipline exhibiting these types of characteristics will contain clear clusters with little collaboration crossing specialization or topical boundaries.

Moody (2004) suggested that the social network model that best fits this scientific disciplinary structure is the small-world model. In essence, a small-world network is constituted by any network where the level of local clustering is high (in this case where 
one's collaborators are also collaborators with each other), but the average number of connections needed to reach other actors is low. Moody (2004) contended, "Distinct research clusters will likely inhibit broad theoretical integration, since theory will profess largely within distinct research groups” (p. 216). Figure 1.2 depicts a graphical representation of how a network characterized by a small-world model might look.

\section{Disciplinary Structural Cohesion Model}

A related model posits that a discipline has little theoretical consensus but is not comprised of distinctively clustered sub-groups. Rather than excluding ideas because of structural barriers characterized by a network of low overall connectivity, this model suggests that the disciplinary structure creates permeable theoretical boundaries. That is, the structure predicates low consensus as a result of a discipline's inability to exclude ideas from the knowledge space once they are introduced (Abbott, 2001). As such, the process of theoretical development is not viewed as linear but instead follows a structure characterized by fractal walks (attempts at exclusion are later diminished because it is often found that those same ideas are reinvented later). As Moody (2004) expressed, "This results in a constant revisiting of ideas and interests in the discipline (though usually from a different direction) as actors continuously loop through wide sections of the available idea space” (p. 217).

According to Moody (2004), the social network model that best fits this description is a model of structural cohesion. A network is structurally cohesive to the extent that ties are distributed evenly across the entire network and is measured by the capacity of a network to remain connected when various nodes are removed from the 
network (Moody \& White, 2003). However, structural cohesion is not only dependent upon the mere connectivity of the members in the group. It is also dependent upon the configuration of the connections through the single actors. That is, structural cohesion is reliant upon the presence of multiple paths, passing through different actors such that, if any one actor is removed, alternative links among members are still present to maintain connectivity (Moody \& White, 2003).

The idea of theoretical permeability allows for and often promotes cross-topic collaboration as the same theoretical frameworks (e.g., economic theories, organizational theories, etc.) can be applied to a variety of empirical questions (Moody, 2004). In other words, although people might specialize in certain techniques (e.g., qualitative methods or social network analysis), they may collaborate freely with others who have different research interests and focuses. In this regard, collaboration patterns across a discipline should be characterized by a structure with few clear divisions or clustering effects. Figure 1.3 illustrates how a collaboration network that is highly structurally cohesive might look.

The Social Construction of Knowledge in the Field of Sport Management Pitts (2001) articulated that a field of study is constituted and gains credibility through many factors including: a body of knowledge and literature pertaining to theory and practice, the engagement of professionals (educators, researchers, and practitioners), and professional organizations dedicated to the advancement of the field. As these are indeed vehicles in which the knowledge generation, transfer, and implementation are 
carried out, they engender a profound opportunity to shape the quality and legitimacy of the activities pertaining to the field of sport management. Incidentally, all three of these factors are characterized by a significant amount of social interaction and influence.

Several scholars in the field of sport management have expressed the benefit and power of scholarly social interaction in shaping and improving the quality of the education, research, and practice of the people and institutions touched by the discipline (e.g., Cuneen, 2004; Frisby, 2005; Pastore, 2003; Pitts, 2001). For example, Pitts (2001) noted how "NASSM has served as a center of activity and a focal point around which those interested in sport management academia have gathered and have been able to share and grow as a family” (p. 5). Daprano et al. (2005) emphasized some compelling reasons to pursue joint research projects with colleagues such as utilizing each other's strengths and sharing ideas. Likewise, Pastore (2003) stressed the need for and value in devoting attention toward mentoring relationships.

As Ennis (1992) articulated, "Making explicit the intellectual and social web of a discipline may shed light on the relative size, prestige, and power of particular specialties, the rise and decline of topical areas, and the diffusion of theories and methods among them” (p. 260). The studies in the sociology of science offer compelling new insights and means to explore the status of the field of sport management from entirely new angles. In challenging the current status of the field from a sociological perspective a whole new range of questions can be raised.

Are the debates and voids that have been plaguing and challenging our discipline reminiscent of some of the very same problems that other disciplines have stumbled through in their evolution and progression? What can the histories and challenges that 
other disciplines have faced offer for us in terms of insight into how to improve our own field? Moreover, are there underlying social structural obstacles that are inhibiting the scientific quality and productivity of studies in the discipline of sport management? If so, what might these structural obstacles hold for the current state and future of research conducted in the field?

From this perspective, an investigation into the social relationships and underlying social structure of the field of sport management offers a wide array of exploratory opportunities. Through the ongoing debates and studies such as Costa (2005) Delphi study and critical evaluations of leading scholars in the field (e.g., Chelladurai, 1992; Chalip, 2006; Cuneen, 2004; Slack, 1998), we have a fairly robust notion of the limitations of our idea space. However, scholars in the field stand to gain great insight by expanding their critiques to include questions about the social interaction and social structures of the people that are doing the studying. 


\section{CHAPTER 3}

\section{METHODOLOGY}

The purpose of this chapter is to describe the methodological procedures implemented to investigate the research questions presented in Chapter 1 . The methods are described in regards to the following aspects of the study: 1) Research Design; 2) Sampling Procedures and Data Collection; and 3) Data Analysis Procedures. Fundamentally, the overarching goal of this study is essentially to explore the question: What do the patterns of scholarly interaction hold for the field of sport management's idea space? More specifically, the research questions that will be addressed include:

1) What is the structure of coauthorship patterns in the field?

2) Do certain variables appear to be related to actors' locations in the overall network structure?

3) What does the collaboration network structure implicate for the transfer of ideas throughout the network?

\section{Research Design}

The methodological premises for this study are grounded in the foundations of social network theory and analysis. As such, the networks of interest are representations of scholarly collaboration which are assumed to fundamentally shape the idea space of 
the field of sport management. Since the focus of this study is on coauthorship patterns, the actors defined for the networks are the scholars contributing to the idea space in the field of sport management (i.e., scholars who have published on sport managementrelated topics).

The data collected was used to describe coauthorship trends in the discipline and construct models of a sport management collaboration network over time. The primary goals of this study were to: 1) explore various network analytic techniques and provide a clear and comprehendible description for future researchers in the field of sport management to employ; 2) identify the observed coauthorship network structure and relate the findings to the evolution of sport management as a field of study; and 3) describe the various implications the results of the study suggest for the transfer of knowledge into idea space in the field of sport management. As participation in the network is a necessity for direct social influence in the network, a model of participation was first derived. Subsequently, the structure of the network among those who have coauthored was then examined through exploratory social network analysis. Finally, qualitative assessments were used to identify thematic patterns related to the underlying social structure of the field.

To explore coauthorship patterns in the discipline, a one-mode (i.e., one type of actor/entity/event of interest) network was used to represent the structure of coauthorship contacts in general. In this regard, the foundational relation of interest was essentially binary and pertained to whether or not two actors in a network have ever co-authored together. The coauthorship network was constructed using the foundational graph theoretical principles of nodes and edges. 
Nodes (i.e., points or vertices) represented the actors, entities, or events of interest in a system and the edges represented relations between any two nodes. Generally speaking, network edges can be defined in an infinite number of ways and representative of undirected (either simply present or not), directional (present from one node to another but not vice versa), or reciprocal (reciprocally directed between two nodes) relations. In addition, ties between nodes can be binary (unvalued and depicted as being present or not) or valued (usually inferring a relative strength or intensity of the tie) (Scott, 2001).

\section{Sampling Procedure and Data Collection}

One of the major threats to the validity of any network study is a researcher's determination of boundary specification for the actors to be included in the analysis (Laumann, Marsden, \& Prensky, 1983; Scott, 2001; Wasserman \& Faust, 1994). Scott (2001) expressed that the extent to which actors who may indeed be relevant to the construct of interest are ignored, the network analyzed will be an imperfect representation of the true underlying structure. Omitting actors who in actuality serve as key people in an analysis can especially distort the overall configuration of structural picture. These issues specifically constitute what network analysts refer to as the boundary specification problem whereby inappropriately defined boundaries render a study subject to partial system fallacy (i.e., a failure to include all of the relevant components of a system) (Laumann et al., 1983). 
In some cases, natural or obvious boundaries may already exist (e.g., an immediate family unit), while in other cases, finding a clear break to bound actors in a network may present a great challenge for the researcher. In fact, logical extensions of even the seemingly evident types of boundary specifications can be easily established (e.g., grandparents, aunts and uncles). Hence, because one of the fundamental premises of a network approach is that the world is comprised of networks not groups, the researcher must be able to reasonably justify the inclusion or exclusion of actors based upon theoretical, conceptual, and/or empirical contentions (Scott, 2001; Wasserman \& Faust, 1994).

Laumann et al. (1983) suggested that, in general, researchers bound their studies in one of two ways. Laumann et al. (1983) distinguished between the two alternatives by calling upon the realist and nominalist views on the ontological status of social phenomena, but note that many network studies do not fall clearly under one category or the other. The realist approach focuses on boundaries set by the actors themselves as membership in the network is based upon their perceptions or recognition of various criteria for belonging to the group. Laumann et al. (1983) expressed, “The realist strategy of setting network boundaries by definition assumes the proposition that a social entity exists as a collectively shared subjective awareness of all, or at least most, of the actors who are members” (p. 21). For example, members of a street gang have insider-outsider "rules" for acknowledgement of group inclusion.

The second way of specifying boundaries highlighted by Laumann et al. (1983), the nominalist approach, is based upon a researcher's artificial imposition of a network 
boundary based upon their theoretical or conceptual interests. Under this approach, Laumann et al. (1983) denoted,

Delineation of network boundaries is analytically relative to the purposes of the investigator, and thus network closure has no ontologically independent status. There is no assumption that reality itself will naturally conform to the analyst's distinction (p. 21-22).

Regardless of whether boundary specification is based off of a nominalist or realist approach, a researcher must somehow enumerate a distinct and finite set of actors to study (Wasserman \& Faust, 1994).

In choosing a set of actors to comprise a network study, researchers generally focus on one or more of three sets of components: actors, relations, or activities (Laumann et al., 1983). Typically, two approaches (and sometimes a blending of both) are utilized when defining a target population: a positional approach and a reputational approach (Scott, 2001). With a positional approach, a researcher samples actors from a formally defined position or group (e.g., members of a sport team). The group or position of interest is identified, a list of all members is enumerated, and then each member is sampled.

When no relevant positions can be identified, and as such no comprehensive list of members is readily available, researchers can implement a reputational approach. With a reputational approach, a common strategy is to construct a list of relevant actors using knowledgeable informants. In this regard, the choice of informants is crucial to the relative validity of the structure generated by the end sample (Scott, 2001). Therefore, it is imperative that the researcher believes the informants have a strong knowledge about 
the target population and are able to report their knowledge accurately (Scott, 2001). Inevitably, there is an element of circularity in this approach because once the network actors are enumerated and subsequently studied, the accuracy of the informants is relatively apparent (Scott, 2001).

A variation of the reputational approach is a snowballing technique whereby a small number of informants are studied and then asked to nominate other informants for the study. These nominees, are then studied, and in turn, asked to nominate still further actors for study. By its very nature, however, a snowball sample is likely to be organized around the connections of the particular individuals who served as the initial informants. Thus, as Laumann et al. (1983) expressed, "It is scarcely informative to learn that a network constructed by a snowball sampling procedure is well connected or 'integrated'” (p. 22).

Although the discipline of sport and exercise management in North America possesses one dominate professional organization (NASSM) which maintains an updated database enumerating all of the current members, the researchers did not believe a positional approach would provide the most valid and accurate means of defining the relevant actors for the network(s) of interest. Instead, we implemented a reputational approach to generate a list of actors cross-referenced with the names identified through an internet search of sport management professors in programs featured by the NASSM website. A panel of experts in the field served as knowledgeable informants to generate a list of productive researchers in the discipline of sport and exercise management. 
The nominated actors were contacted and asked to supply an updated copy of their curriculum vita. In addition to the request for their curriculum vita, the nominees were also asked to complete a six-item questionnaire. As one of the focuses of this study was an exploration of the coauthorship patterns, and most curriculum vitae provide a detailed listing of a person's publications, further triangulation of the names generated was carried out by cross-referencing the coauthors listed on the collected curriculum vitae to the overall listing of the population. Ideally, between the names generated by the panel of experts, the listings of the NASSM membership database, and the coauthorship listings from the nominated individuals' curriculum vitae, a relatively comprehensive network of actors was believed to be clearly defined. Even so, to further supplement the data, the articles from the top five journals in which the participants most frequently published were added to the dataset

After three weeks, a thank you letter was sent out to respondents and a reminder request was sent out to all non-respondents on the list. In addition, the data collected from the first round of curriculum vitae revealed new actors for the network (i.e., co-authors listed that are not already on the list), and an attempt was made to contact these new nominees to elicit their participation. One week after the second requests were sent out (or the first request for any new nominees), thank you letters were sent to all new respondents.

The classical issues pertaining to the reliability and validity of a study are invoked in a somewhat different manner in network analytic approaches as compared to traditional quantitative methods. In essence, due to the fact that network analysis is still a relatively new and developing methodological approach, the exact relevance of these 
issues to network analyses has not been resolutely determined and formalized (Wasserman \& Faust, 1994). Therefore, handling these issues for this study falls more along the practices of qualitative research in that the researcher seeks accuracy through as much transparency and robustness as possible in terms of how the data is collected, analyzed, and tied to the propositions being made. For example, reliability and validity checks in network studies often take the form of triangulating the information obtained with multiple approaches to investigation (e.g., asking actors’ perceptions as well collecting data on actual observations).

In general, a measure of a concept or variable is considered reliable to the extent that repeated measurements give the same estimates of the variable. In standard psychometric testing instruments, the reliability of a measure can be assessed by comparing measurements taken at two points in time or by comparing measurements based on subsets of test items. Fundamentally, the idea of a test-retest assessment of reliability in network studies is not likely to be appropriate because one must assume that the "true" value of a variable has not changed over time. Ontologically, a network perspective holds that social phenomena cannot be "assumed to remain in stasis over any but the shortest spans of time” (Wasserman \& Faust, 1994).

The validity of a study is concerned with the extent to which a measure actually measures what it is intended to measure. Since many network approaches, and specifically the approach used in this study, entail a sort of census sampling of a population, the notion of external validity (i.e., the extent to which the results of a study can be generalized to a population) becomes somewhat obsolete. Likewise, as the aim of this study is more descriptive in nature than looking for causal relationships per se, the 
determination of the internal validity (i.e., degree to which observed differences on the dependent variable are directly related to the independent variable, not to some other uncontrolled variable) of the measure is also not of particular concern.

In essence, because the overarching concept of interest is ultimately the social structure of the discipline, the main threats to the overall validity and reliability of the study that must be addressed include: 1) how the target population is defined and sampled and 2) determining relevant relational ties that can accurately depict or represent the underlying social structure. The first threat was previously addressed in the section describing the sampling method for the study. By using a reputational approach and cross-checking the nominations of the informants with the institutions identified by the NASSM website, the snowball coauthorship records obtained from curriculum vitae, and the information obtained from the journals ultimately exhibited a relative circularity whereby the names generated from each of the methods overlap. If this is the case, which indeed it was, the dataset should be a relatively robust and valid sample of data.

The second threat has not yet been thoroughly addressed and pertains to the relations that were used to represent the ties connecting the actors in the underlying social structure. The use of curriculum vitae as the primary means for data collection offers a wide variety of exploratory opportunities for studying social factors in scientific knowledge production. For example, many researchers in the field list the institutions where they completed their doctoral work, and in many cases, their doctoral advisor on their curriculum vitae. Likewise, many researchers also include a statement of research interests or specialty focuses. 
For the primary purposes of this study, the fact that researchers almost always provide a comprehensive listing of their publications over the course of their academic careers was of particular value. As the main focus of this investigation was to construct a collaboration network based upon coauthorship on publications, the curriculum vitae served as a direct means for obtaining information on the papers a particular researcher has published. In addition, the listing of publications also served as a record of all of the formal collaborations the researcher has engaged in over the course of their career.

Since the evolution of a collaboration network can be heavily dependent on the passage of time, time variables were observed and incorporated into the study. As 1985 was the year in which the beginnings of a formal organization of the field began through the creation of NASSM, the publications included will be focused on those published in 1985. Publications generated through curriculum vitae that were written prior to 1985 were included, however, the time block was simply labeled as "prior to1985" during the analysis process. The rest of the publications were blocked in five-year increments (with the exception of the block of time still currently in progress): 1985-1989, 1990-1994, 1995-1999, 2000-2004, 2005-In Press.

As was mentioned earlier, in addition to sending their curriculum vitae, subjects were also asked to fill out a six-item questionnaire. The four items included an openanswer format to the following statements: Please list the names of the top five people in the field of sport management 1) with whom you are most likely to collaborate on peerreviewed publications; 2) with whom you most frequently discuss research-related topics; 3) whom you believe to have greatly influenced your work (this last one does not have to be someone you interact with personally such as a specific researcher whose work you 
often cite); 4) the name of your doctoral committee chairperson (advisor). In addition, the participants were asked two items related to the substantive areas their research has covered.

Fundamentally, the point of these questions were to provide a basis to compare the measures of the formal communication patterns obtained from actors' curriculum vitae (i.e., publications) with actors’ perceptions about their collaboration and other informal communication patterns. In essence, the responses to this brief questionnaire helped provide a general sense of the validity and reliability of the formally recognized patterns of interaction provided by the co-authorship data with some information about informal communication patterns. Collectively, these two measures provide a robust depiction of the underlying social structure of the discipline of sport and management.

\section{Data Analysis}

Although there are a number of relational patterns that could potentially serve as conduits for the flow of information in a disciplinary network, the types of ties and resultant networks that were explored in this study were selected based upon theoretical and conceptual propositions as well as the information that was made available through the sampling method. Specifically, the data collected was analyzed relative to coauthorship network patterns, institutional affiliation patterns, student-advisor relationships, time in the network, specializations, methodological preferences and refereed publication productivity. In addition, the constructed networks were analyzed relative to distinct time-blocks in order to grasp an understanding of how the network structures evolved over time. 


\section{$\underline{\text { General Collaboration Trends }}$}

The first part of the analysis pertained primarily toward assessing the general collaboration trends in the field of sport management for each of the designated time blocks. We looked at such trends as the growth in number of actors in the network and number of publications over each of the time blocks. We also analyzed the number of publications per author and number of authors per paper over time. Finally, to draw some conclusions as to who were the most active members in the network and those who had only published one or two times, we qualitatively explored the actors at both extremes of the distribution for number of publications per author.

\section{Visualization and Network Evolution}

As one of the most helpful tools provided by social network is the ability to use graphical representations of the network to aid in interpretation, we heavily relied upon network visualization techniques in interpreting the results of the study. In particular, we employed visualization to help tell us the story of the evolution of the network over each of the designated blocks of time. In doing so, we were able to qualitatively explore various features of the network and develop several themes pertaining to the attributes of the actors and the obtained network structure.

\section{Quantification and Property Exploration}

As visualization can be limited in terms of interpretation, particularly for the larger and more complex structures, we also employed various analytical procedures available through the software program Pajek to explore a number of quantification 
measures. For example, we explored patterns of connectivity through the assessment of vertex degree values, $k$-core identification, and clique extraction. We also investigated measures of closeness and betweenness centrality to identify structurally important actors in the network, clustering patterns, the strength of ties between actors, and actors' patterns of structural holes.

\section{Thematic Development}

Finally, in the later stages of the analysis process, we created several network partitions based upon the emergent themes in the earlier stages. These partitions included: 1) regional institutional affiliations, 2) affiliation with one specific institution, and 3) methodological preferences. All three of these partitions were assessed qualitatively and quantitatively in terms of how they related to the centrality properties of the observed network as well as how they could potentially impede or facilitate the diffusion of an innovation throughout the network. 


\section{CHAPTER 4}

\section{RESULTS}

The analysis for this study employed exploratory social network analysis only. That is to say that the researchers had no specific hypotheses about the structure of the network ahead of time that were tested per se. As no research looking into the collaboration patterns in the field of sport management has been done before, it was difficult to generate testable statements. Therefore, for the initial analysis of this data, the researchers adopted an exploratory approach which was used to explore the network(s) for meaningful patterns. In addition, rather than analyzing samples of the network (which might be construed as the population under conventional methods), the entire observed network was analyzed using articulated boundary specifications. Under these premises several themes were developed to lay the groundwork for future studies.

\section{Executive Summary}

As the data collected for this study far exceeded the scope of a single report, the results presented in this chapter are intended to essentially tell a story of the evolution and current state of the field of sport management's coauthorship patterns. The design of this chapter was developed to provide the reader with a relatively intuitive and 
transparent step-by-step understanding of how the descriptions of the structure were obtained. In addition, throughout the description of the analyses performed and the subsequent results, several emergent themes are highlighted. It is the goal of the researchers to develop these themes to the point that they can serve as springboards for future testable hypotheses related to the social structure and idea space of the field of sport management.

\section{Sampling Procedures}

\section{Stage 1}

The initial list of names generated by the panel of experts and supplemented by a mass internet search for email addresses of sport management instructors consisted of a total of 254 names and email addresses. Once the list was generated, an email was sent to each of the 254 addresses with an official cover letter and a questionnaire attached. Despite several attempts and checks verifying the correctness in spelling, the emails to four of the addresses continually failed to send. Upon further investigation into those names, it was discovered that two of them had retired from academia and two of them appeared to have either moved to other institutions or retired from academia.

The first round of sampling produced 51 responses including both the participants’ curriculum vitae and their completed questionnaires. Three additional participants sent only their curriculum vitae but no questionnaire and one participant returned the questionnaire but did not send his/her vita. In addition, 24 people responded that although they teach in the field of sport management, their contract loads do not require and/or allow them to spend time publishing; seven people responded noting that 
although they publish research articles, they do not consider themselves to be active in the field of sport management; and 13 replied expressing they did not have time or did not wish to participate in the study. In total, of the initial 254 names generated, responses were received from 98 people. As some people from the initial list were identified as not qualified, an adjusted desired response total was recalculated as 223 (i.e., 31 responses noted not qualified subtracted from the 254 names generated). Therefore, the response rate for the first round was calculated as $22.87 \%$ complete responses with an additional $2.47 \%$ when the 4 partial responses are included.

\section{$\underline{\text { Stage } 2}$}

Once the first round of responses was obtained, all of the publications from refereed journals listed in the vitae were put into a spreadsheet with the complete listings of all of the author names. A thorough web search was conducted on each of the coauthors to determine if any names of people who regularly carry out research related to the field of sport management had been missed in the first name generation stage. Names of authors who were identified from programs outside of the United States and Canada or of individuals who were researchers whose primary research focuses had little to do with sport management were not included as new participants. In total, 24 new names of authors who were likely to be sport management-focused researchers were identified.

Three weeks after the initial emails were sent, a reminder email was forwarded to all those addresses from which no response had been received. In addition, the 24 new authors that had been generated were each sent the original email, cover letter, and questionnaire asking for their participation. The same process was implemented with the second round of sampling where all of the new names generated were investigated to 
determine if any new names had been identified as prominent contributors to the literature related to sport management. As no new names were identified through the process, the sampling of the second round appeared to exhaust the identification of new names to sample.

The reminder email and second generation of names produced 21 more curriculum vitae, 19 of which included questionnaires. Thus, the total response count from both rounds was 71 comprehensive responses (i.e., vita and questionnaire both returned), 6 additional curriculum vitae, and one questionnaire only response. In addition, 13 more people responded expressing that their teaching loads do not permit them to conduct research, 5 responded noting that their research focus is not sport management, and 8 responded that they did not have time and/or did not wish to participate in the study. Recalculating the total desired responses to be 229 as reflected by those expressing that they did not qualify and the new names generated from the first round of sampling, suggested an adjusted response rate of 31\% for completed responses and an additional $3.06 \%$ with partial responses.

Of the total number of curriculum vitae collected (i.e., 77), two were later identified as not qualifying for the study as they had not published in refereed journals and three were received after the end collection date when the analysis had already started. While the information about the authors whose vitae had arrived late was included in the latter half of the study in terms of identifying themes and trends explaining collaboration patterns (e.g., institutional affiliation), several of their publications may not have been included in the coauthorship network; particularly if the publication was one of single authorship. The three additional vitae were later analyzed 
and shown to add only seven new ties to the unique coauthorship network and ultimately did not significantly change the rest of the results.

\section{$\underline{\text { Stage } 3}$}

Prior to the analysis stage, a list of all of the publications in refereed journals was extracted from participants' curriculum vitae and put into a spreadsheet. After the input was completed, the researchers identified the top five journals in which the participants' most often published their work. These journals included: The Journal of Sport Management, The Journal of Legal Aspects of Sport, Sport Marketing Quarterly, Sport Management Review, and European Journal of Sport Management (which in later years changed to European Sport Management Quarterly).

Although a couple of journals were also identified as journals where sport management related articles are frequently published such as Strategies and the Journal of Physical Education, Recreation and Dance, the researchers determined that because these journals also tend to attract a lot of articles from scholars in other fields, particularly physical education, including these journals in the next round might capture too many actors from a related but separate field of study. If a sixth journal were to be identified, The International Journal of Sport Management would have been the next choice. However, due to the challenge of getting access to the journal, the researchers chose not to carry this journal into the next step of the data collection.

To supplement the publications and list of authors obtained through Stages 1 and 2 of the data collection process, the researchers added all of the publications and their authors from the five identified journals that had not previously been identified from participants' curriculum vitae into the spreadsheet. With the exception of three issues of 
European Sport Management Quarterly (due to an inability to access them), all of the articles published in the journals from the earliest issue to the most recent issue were included in the spreadsheet. While a number of new names were identified during this process, most were authors who had published only once, worked at institutions outside of the United States and Canada, and/or mainly conducted research in other fields (e.g. economics or business) but happened to carry out a study related to sport management. Nonetheless, as was hoped for and expected, the names and publications generated in the previous steps and the names and publications found in the five journals overlapped a great deal.

\section{Stage 4}

Once the total list of publications and actors was completed, an additional spreadsheet was created holding a variety of accessible attribute information on each of the authors (e.g., institutional affiliations, terminal degrees, graduate institutions, and doctoral advisors). As the curriculum vitae and questionnaires provided much of this information, a great deal of attribute information was generated for 76 of the actors. To identify information on more of the actors in the study, the journal articles used often provided such information as institutional affiliation and sometimes where the authors earned their degrees (particularly if the article was published while the student was still completing his/her degree). Even so, the information provided on the attributes was rather sparse, and therefore, the spreadsheet on actor attributes contained a lot of missing data particularly for those who were identified as individuals who had only published once in the field. 
As the vitae generated the most robust attribute information for the actors, the actors in the network who had submitted their information consequently served as a primary source of the data for generating themes for future investigation. Using the responses from the questionnaires, each participant was coded as primarily implementing qualitative methods, quantitative methods, both qualitative and quantitative, or legal/case law analyses. The researchers also looked at years in the field as calculated based upon the year in which the participant's first work in a refereed journal was published as well as the total number of refereed articles the participant had published.

The specializations listed were derived from a coding of the self-identified item on the questionnaire asking participants to describe the primary focuses of their research. In order to capture a broader scope of specializations, five broad specializations were used: 1) sport management which included such special focuses as leadership, organizational behavior, organizational theory and human resource management; 2) sport marketing which included such special focuses as consumer behavior, sponsorship, donor motives, and spectator behaviors; 3) sport law which included those participants who possessed law degrees as well as those who had published on such topics as risk management, case analyses and law reviews; 4) sport sociology which included those who noted that much of their research had pertained to social and diversity issues and sport; 5) physical education which encompassed all those whose terminal degree actually fell in this category and early works tended to entail a physical education focus; 6) business which included authors whose terminal degrees were in such fields as general business, economics, marketing or management and published articles related to these fields in addition to sport-related topics; and 7) other which comprised of all other 
specializations that did not fall under one of the six previously identified categories. If a questionnaire was not sent along with the vita, a specialization was coded as falling into areas under which the titles of their publications and the journals they published in appeared to indicate. In order to capture the overlap between specialties, authors were not limited in the number of specializations to which they were tied.

To protect the identities of the participants, institutional affiliations were coded into different columns (labeled A, B, C, D, E, F, etc.). A “1” listed in the column under a particular heading indicated that the participant, at some part of their academic career, had been affiliated with the institution (including their graduate studies). In addition, a separate column identified the school where their highest terminal degree was obtained using the same coding system. Each doctoral advisor listed in participants' questionnaires was given a pseudonym to protect the anonymity of the individuals and were also included in the spreadsheet.

In total, the participants in the study possessed affiliations with 99 different universities all over the world including: 83 schools located in the United States, 9 schools located in Canada, and 7 schools outside of the United States. Details related to years in the field, number of publications per participant, and number of unique coauthors per participant can be found in Appendix A.

As highlighted in Chapters 1 and 3, the limitations of any network analytic study are inextricably linked to boundary specifications for the inclusion and exclusion of actors in the analysis. Because social network analysis inherently embraces the idea that the world is comprised of a networks of networks, an underlying assumption of any study is that the actual number of actors and their relationships in a network may never be 
concretely determinable and the types of ties between actors under investigation may be too complex and overlapped to ever fully capture. Therefore, before discussing the results and implications of the study, it is first necessary to address the representativeness and relevance of the data collected.

In order to build robustness and aid in the validity in terms of identifying relevant actors for the network under investigation, several sampling and data collection techniques were implemented. In the first stage of the data collection a name generation round ensued in which a panel of experts consisting of five sport management professors from research-intensive universities was asked to identify scholars that they know publish in the field of sport management. To eliminate the possibility of capturing scholars affiliated with one particularly institution, two members of the panel of experts had no direct institutional affiliation ties with the institution in which the primary investigators were affiliated. In total, the panel of experts possessed institutional affiliations with ten different universities, nine of which are research-intensive.

In addition, to ensure that the names generated did not overlook actors who publish in refereed journals but whose institutional affiliations mainly consist of teaching colleges and universities, a thorough web search for potential sport management researchers was conducted on the websites of universities identified by the NASSM website as offering sport management degree programs. Furthermore, once the first round of responses was received, a snowballing technique based upon the coauthors of the participants' was used to further alleviate the possibility of missing any other individuals 
that may play an important role in terms of contributing to the idea space of the field. As such, it is assumed that the data collected and used to obtain an observed network structure was relatively robust and representative of the actual network structure.

\section{Analysis}

To examine network participation and embeddedness, the list of all publications and their authors generated from both the curriculum vitae collected and the five supplemental journals were coded into a SAS file whereby each author's name was translated into a unique identification number. In addition, the spreadsheet with the authors' attribute information was coded with corresponding identification numbers such that the author's identification number in the SAS file matched the authors' identification number in the attribute spreadsheet. In total, 2,080 publications were included in the network producing a list of 1,494 unique author identification numbers.

As names can be a problem due to inconsistencies over time (e.g., marital status or inconsistent use of middle initials), an attempt was made to ensure that the author names represented a unique actor in the system. For example, the researchers assumed that an author listed in bibliographical references as "Smith, J." with very similar coauthors and/or institutional affiliations as "Smith, J.R.” represented the same person. Likewise, an attempt was made to distinguish those who may have changed their name over the course of their careers. Although it is possible (and indeed likely) that some names were missed, due to the relatively small size of the overall generated network (in comparison to other fields of study) and the robustness built between the collection of the 
curriculum vitae and the journals, the researchers do not anticipate that there are significant differences between the combinations of the inconsistencies in the observed network structure and the actual network structure.

\section{Exploration of General Collaboration Trends}

Before looking at the structural patterns related to coauthorship, it is useful to first look at some of the general collaboration trends. Two primary constraints on the shape of a collaboration network are the distributions of the number of papers people publish and the number of authors who collaborate on a single paper. According to Moody (2004), a low number of authors per paper, decreases the size of the complete clusters formed through common authorship on a single paper. Table 4.1 provides distributions of the number of authors per publication, and Table 4.2 shows the distributions of the number of publications per author. Each designated time block only counts publications within that period. However, authors may have published in multiple time blocks. As such, the total column is not the sum of all time periods but rather gives an indication of the collaboration trends of all authors over all blocks of time. 


\begin{tabular}{rrrrrrrr}
\hline Count & \multicolumn{1}{c}{ Total } & Pre-1985 & \multicolumn{1}{c}{$1985-$} & \multicolumn{1}{c}{$1990-$} & \multicolumn{1}{c}{$1995-$} & $2000-$ & \multicolumn{1}{c}{ 2005-In } \\
\hline 1 & $30.00 \%$ & $38.37 \%$ & $37.68 \%$ & $44.58 \%$ & \multicolumn{1}{c}{1999} & \multicolumn{1}{c}{2004} & \multicolumn{1}{c}{ Press } \\
2 & 41.73 & 43.02 & 38.41 & 34.64 & 44.07 & $25.51 \%$ & $19.14 \%$ \\
3 & 19.38 & 11.63 & 18.84 & 17.78 & 19.77 & 19.40 & 41.41 \\
4 & 5.63 & 5.81 & 6.10 & 2.12 & 4.14 & 7.33 & 9.38 \\
5 & 2.12 & 1.16 & 1.45 & 0.60 & 1.51 & 2.44 & 5.08 \\
6 & 0.67 & 0.00 & 0.00 & 0.00 & 1.51 & 0.54 & 0.78 \\
7 & 0.10 & 0.00 & 0.00 & 0.00 & 0.00 & 0.27 & 0.00 \\
8 & 0.19 & 0.00 & 0.00 & 0.00 & 0.00 & 0.54 & 0.00 \\
9 & 0.10 & 0.00 & 0.00 & 0.00 & 0.00 & 0.14 & 0.39 \\
10 & 0.05 & 0.00 & 0.00 & 0.00 & 0.00 & 0.00 & 0.39 \\
11 & 0.05 & 0.00 & 0.00 & 0.30 & 0.00 & 0.00 & 0.00 \\
$\mathrm{~N}$ & 2080 & 86 & 138 & 332 & 531 & 737 & 256 \\
\hline
\end{tabular}

Table 4.1 Distributions of Authors per Publication for Each Time Block

\begin{tabular}{rrrrrrrr}
\hline Count & Total & Pre-1985 & $\begin{array}{r}1985- \\
1989\end{array}$ & $\begin{array}{r}1990- \\
1994\end{array}$ & $1995-$ & $\begin{array}{r}2000- \\
2004\end{array}$ & $\begin{array}{r}2005- \\
\text { In Press }\end{array}$ \\
\hline 1 & $65.33 \%$ & $66.13 \%$ & $73.94 \%$ & $69.76 \%$ & $70.58 \%$ & $65.31 \%$ & $66.98 \%$ \\
2 & 13.72 & 11.29 & 11.97 & 11.68 & 14.26 & 13.27 & 17.30 \\
3 & 5.82 & 4.84 & 4.23 & 6.19 & 4.15 & 6.12 & 5.03 \\
4 & 3.82 & 4.84 & 2.11 & 3.78 & 2.71 & 3.50 & 2.83 \\
5 & 1.67 & 1.61 & 2.11 & 2.06 & 1.62 & 2.62 & 1.89 \\
6 & 1.54 & 3.23 & 0.00 & 0.69 & 1.26 & 2.77 & 1.89 \\
7 & 0.94 & 1.61 & 1.41 & 0.69 & 0.72 & 1.46 & 0.63 \\
8 & 0.8 & 1.61 & 1.41 & 1.72 & 0.72 & 0.73 & 0.31 \\
9 & 0.6 & 0.00 & 0.00 & 0.34 & 1.26 & 0.29 & 0.94 \\
10 & 0.94 & 1.61 & 0.00 & 0.69 & 0.72 & 0.15 & 0.31 \\
11 & 0.27 & 0.00 & 2.11 & 0.34 & 0.00 & 0.44 & 0.63 \\
12 & 0.47 & 0.00 & 0.00 & 0.34 & 0.36 & 0.15 & 0.31 \\
$>12$ & 4.27 & 3.23 & 0.70 & 1.72 & 1.62 & 3.21 & 0.94 \\
$\mathrm{~N}$ & 1,494 & 62 & 142 & 291 & 554 & 686 & 256 \\
\hline
\end{tabular}

Table 4.2 Distributions of Publications per Author for Each Time Block

One trend indicated by the dataset is simply the dramatic increase in the number of papers being published as well as the number of authors producing work in the field as 
shown from the increase from about 82 authors for 86 publications prior to 1985 to about 686 authors for 737 publications in the time period from 2000-2004. Incidentally, as Table 4.2 indicates, according to the dataset about $65 \%$ of all authors that appear in the network have published only once and about $14 \%$ more have published only twice in the field. In addition, according to the results, the number of single-authored papers dropped from about 38\% of all papers prior to 1985 to about 25.5\% of all papers from 2000-2004. Although the block of time labeled 2005-In Press is still in progress, it appears that the decrease in single authorship on publications may continue. Moreover, along with the increase in the proportion of papers that are being coauthored, there also appears to be a slight general increase in the number of coauthors per paper.

To draw some connections as to whether or not there are specific attributes of individuals that could possibly be correlated with their productivity in the field, the actors who fell in the extreme parts of the distribution (i.e., authors with lots of publications or authors with only one publication) for all time blocks combined were explored in greater depth. The information from the actor attribute spreadsheet (both the information from the participants as well as the information extracted from the journals) was used to identify certain themes. As such, it is important to note that the results are not conclusive but rather themes that were drawn from looking at the accessible data.

Some of the apparent themes for those actors who were identified as only having one publication included: 1) graduate students publishing with an advisor; 2) practitioners as a single author or collaborating with scholars in the field; 3) scholars in the field of sport management whose institutions were not located in the United States or Canada; 4) scholars who likely publish in another field but happened to submit an article in one of 
the journals or collaborate with a sport management scholar for a particular article; and 5) scholars who have only recently entered the field (i.e., recently completed their graduate studies).

To look at attributes for those who had published the most articles in the field, only those authors with more than ten publications were included in the analysis. In total, 72 authors were identified as having 11 or more publications. Some themes that emerged from this group of actors included: 1) most either currently work at or have previously worked at a research-intensive university and 2) many actors that have been affiliated with several select institutions. While it was expected that those who had published the most in the field, were likely to have also been in the field for a longer period of time, this was not necessarily the case. In fact, several scholars who had been in the field for fewer than five years had not only matched the number of publications of people who had been the field for 20 or more years, but had actually surpassed quite a few of them.

As a check for the robustness of the sample, the researchers also examined how many of the actors who were identified as having published 11 or more times had served as participants in stages one or two of the sampling. In total, 28 out of the 72 actors had not been participants while 44 actors had responded with their curriculum vitae. Of those with 20 or more publications, 32 out of the 34 actors sent in their curriculum vitae. Incidentally, two of the individuals whose curriculum vitae arrived too late to be included in the analysis for unique coauthorship, made the list for those who had produced over 11 publications indicating that the overlaps of all of the sampling stages provided a relatively robust sample of publications. 


\section{Exploration of the Coauthorship Network Structure}

While the indices above provide us with information about general trends in the volume of publications, publications per author, and the number of coauthors per publication, they do not provide us with any information about the trends of who coauthors together and how often they coauthor together. Under conventional statistical methods, it is difficult to capture the information necessary to answer these questions. Thus, a social network approach is beneficial for analyzing the dataset in these regards.

In performing analyses on social networks, it is often constructive to first use special software programs to draw the network for you. Network visualization is considered to be a great tool for providing an intuitive interpretation of the general network structure. The drawing software typically uses nodes (points or vertices) to represent actors in the system and edges (or lines) between the nodes to represent the relations among the actors. In essence, a network picture is simply a graphical representation of the ties and actors that are under investigation.

\section{$\underline{\text { Visualization }}$}

For the initial visual analysis in this study, the coauthorship network (i.e., the ties between all actors generated) for all publications and all time blocks was drawn using the software program Pajek under the Fruchterman Reingold 2D layout. Upon initial inspection, it is clear there are a number of authors who are isolates or outliers from a larger connected main network; some who have never coauthored (which appear as a single node with no ties to any other nodes) and some who have coauthored with others but collectively are not tied to the larger connected network group (which appear as 
smaller groups of nodes tied together but not tied to the larger network group). Figure 4.1 provides an illustration of the picture obtained through this process.

While this information is interesting in and of itself, it is also useful to consider how the intensity between authors (i.e., strength of tie as calculated by the number of articles they have published together) looks when built into the global network picture. Figure 4.2 provides an illustration of the global network with the strength of ties between authors included. The thickness of the lines provide a visual image of the intensity between two actors and the numbers next to the lines actually indicate how many times the two authors have published together. An initial inspection tells us that there are a number of ties throughout the global network with a range of intensities.

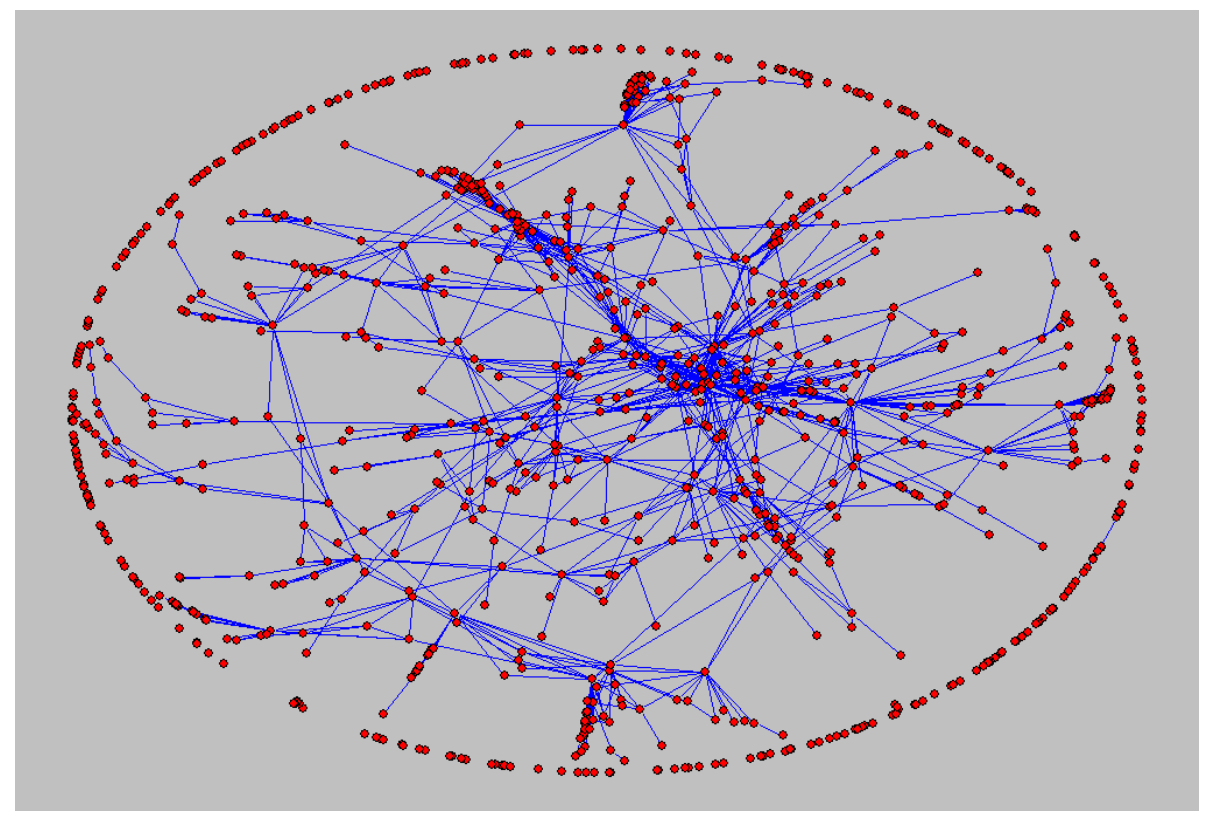

Figure 4.1 Global Network Illustration of Sport Management Unique Coauthorship Ties 


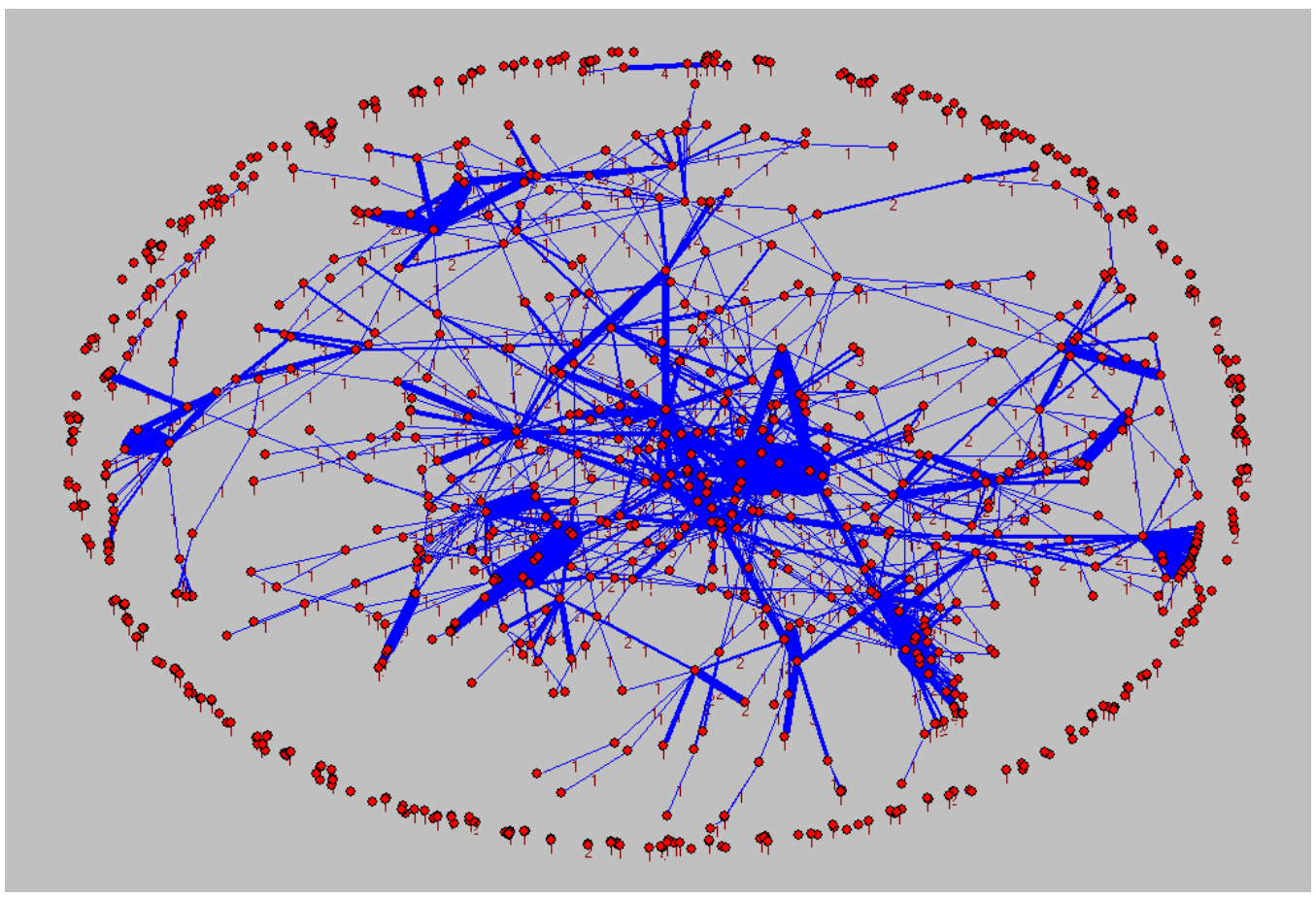

Figure 4.2 Global Network Illustration of Coauthorship Tie Strengths

As we are also interested in the development of the coauthorship network over time, it is beneficial to look at the global illustration of each of the designated time blocks both in terms of a visual structure of unique ties and the intensity of ties. Figures 4.3, 4.4, 4.5, 4.6, 4.7, and 4.8 provide these images for the time blocks prior to 1985 , 1985-1989, 1990-1994, 1995-1999, 2000-2004, and 2005-In Press respectively. To aid in the visualization, the networks were partitioned (i.e., categorized) into components (i.e., actors connected by a chain of ties of any length). Each of the figures represents only those publications released in that time period thus the unique ties and strength of ties between actors are not carried over from one image to the next. 


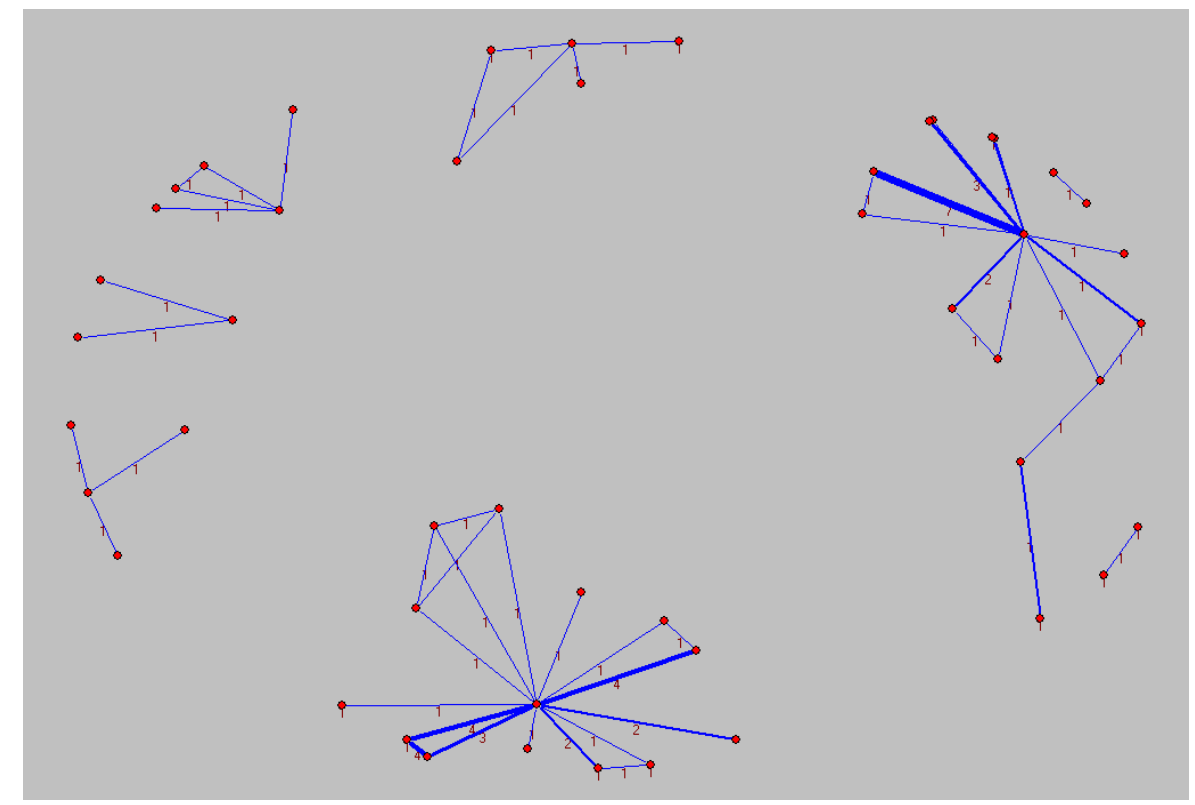

Figure 4.3 Global Network Structure of the Intensity of Coauthorship Ties Prior to 1985

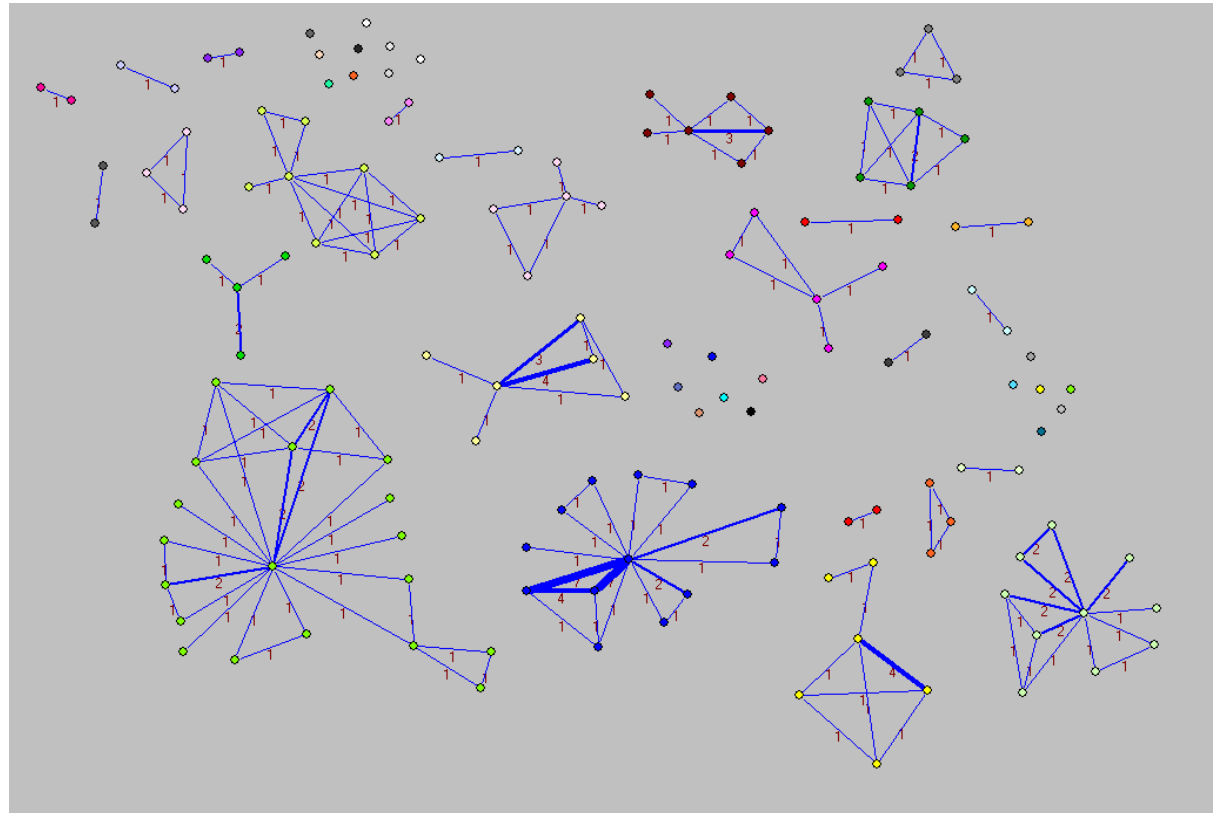

Figure 4.4 Global Network Structure of the Intensity of Coauthorship Ties 1985-1999 


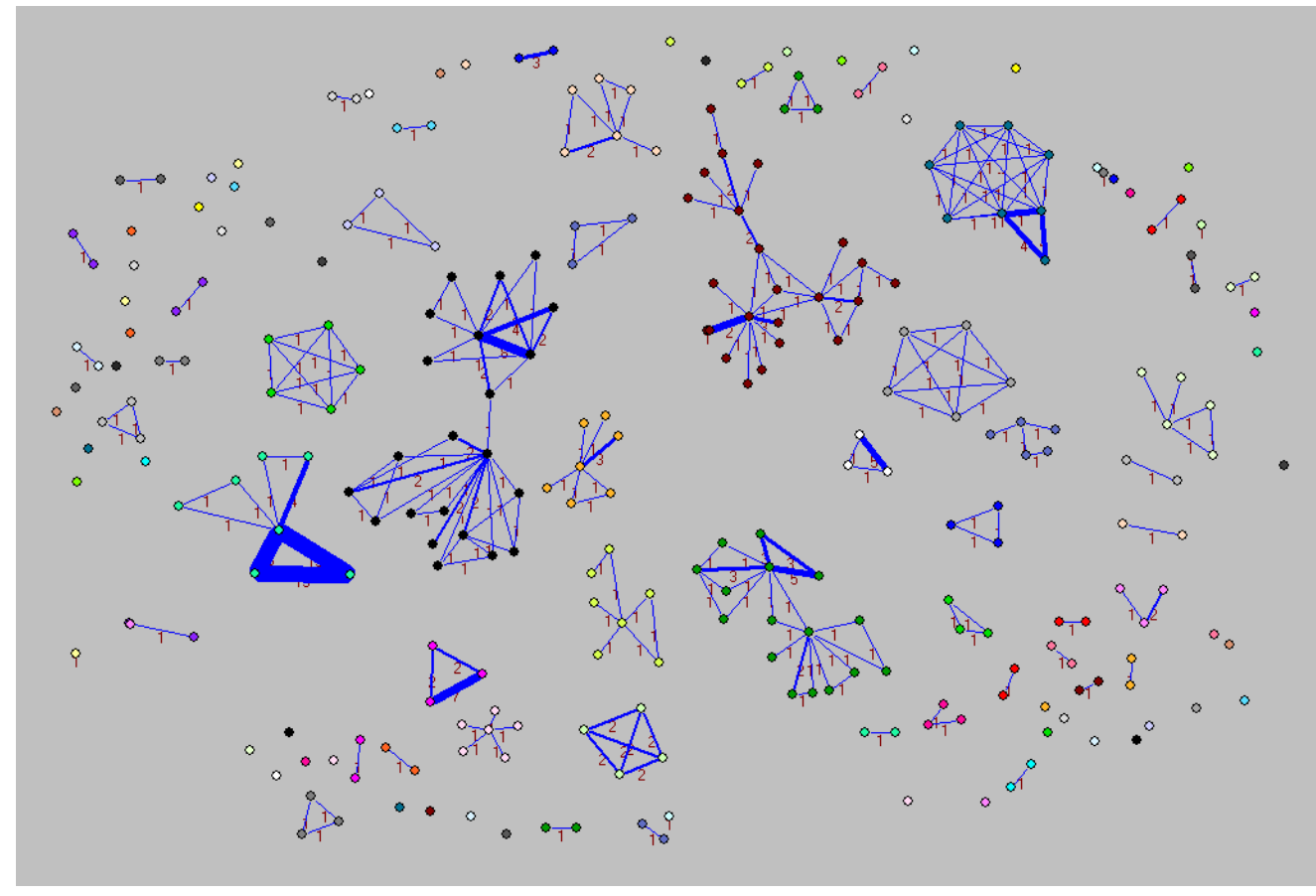

Figure 4.5 Global Network Structure of the Intensity of Coauthorship Ties 1990-1994

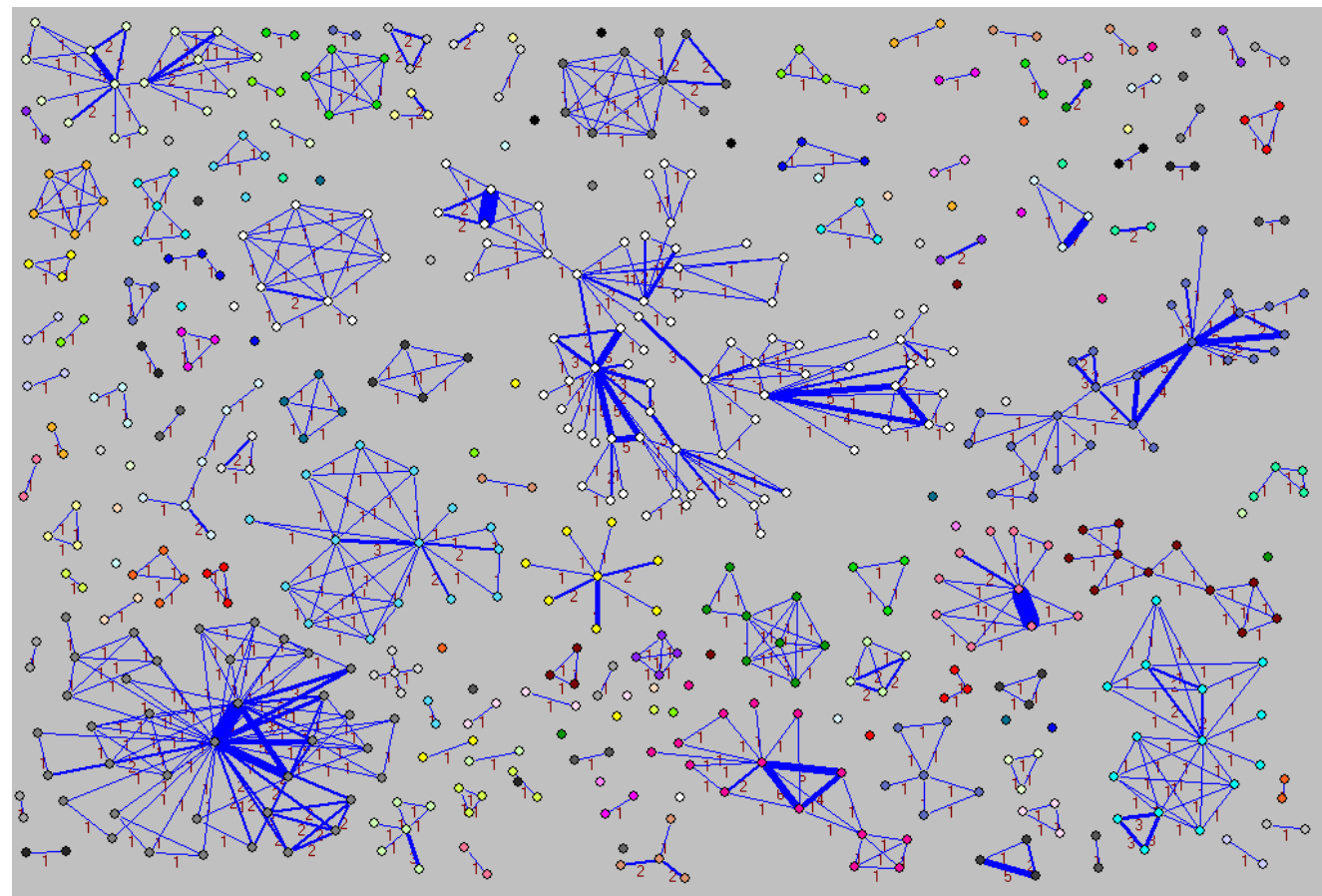

Figure 4.6 Global Network Structure of Intensity of Coauthorship Ties 1995-1999 


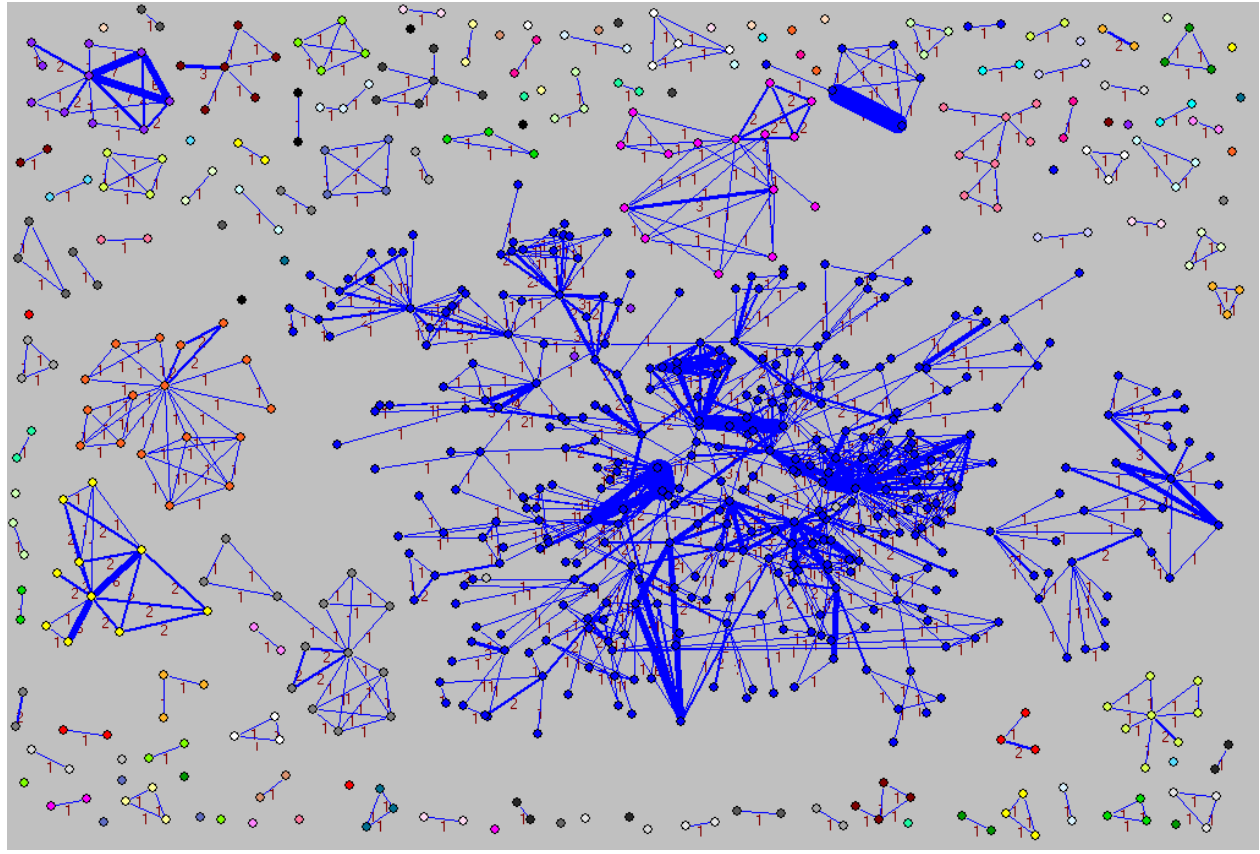

Figure 4.7 Global Network Structure of Intensity of Coauthorship Ties 2000-2004

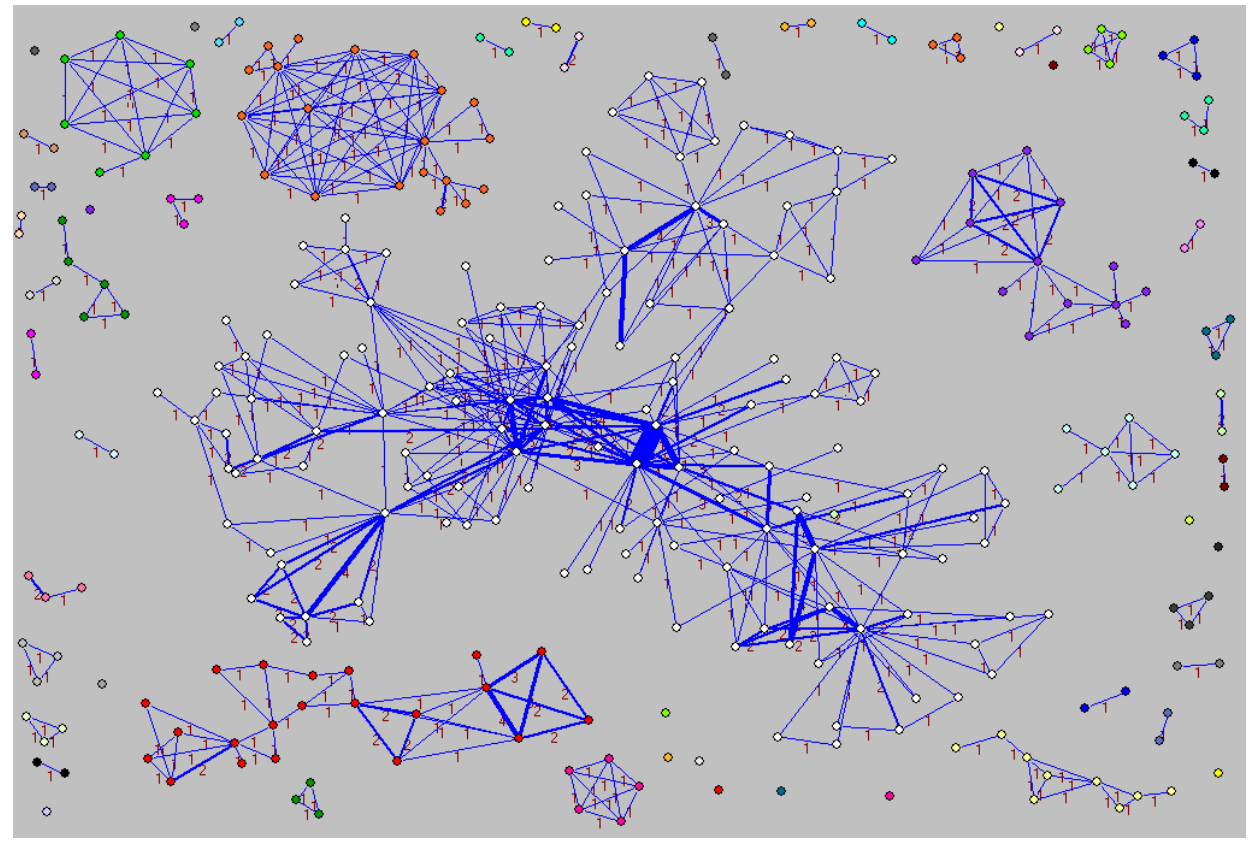

Figure 4.8 Global Network Structure of the Intensity of Coauthorship Ties 2005-In Press 
In addition to the sheer growth in the number of authors involved in the field, as we progress through each block of time (with the exception of 2005-In Press as this represents the current time block and is only indicative of about half of time period of the other blocks), we can make some initial observations about potential structural coauthorship trends. For example, in the years prior to 1985, there are several very distinct groups of connected actors. In addition, we can see that every actor collaborated with at least one other person at least one time. Visualization also allows us to identify the author identification numbers that each vertex represents. Thus, we can also draw some connections between the structural positions of the nodes and the attributes of those actors the nodes represent.

As such, the attributes for the actors in the obtained network for the field prior to 1985 suggest that each of the separate components is comprised of a somewhat unique set of collaborators. One of the two larger components appears to consist of scholars from several different universities in Canada with backgrounds primarily in physical education and a couple of individuals with specializations in sport psychology and organizational behavior. The second of the two large components is characterized by a group of scholars with backgrounds in business management and marketing primarily from a couple of different institutions in the western part of the United States. The smaller components in the network appeared to include: 1) several groups of scholars from various institutions in the United States with physical education backgrounds; 2) one group of scholars located in the United States with business backgrounds; and 3) one group of scholars from a couple of different institutions not located in either the United States or Canada with backgrounds in physical education, sociology, and public policy. 
In the visualization picture for the years 1985-1989, some similar observations can be made in that a number of very distinct connections of actors are present albeit quite a few more of them. However, we can also see a few isolates (actors who in this time period did not coauthor with anyone else in the network). Upon further exploration, two of the larger components from the previous time block appear to have a minimal carry over in that many of the individuals who had collaborated together in the previous time block were again connected in this time block. The component comprised mostly of scholars from Canada also gained a few new actors from several different international institutions by way of publishing one article with one of the scholars from a Canadian institution. Several actors from the second larger component from the previous time block also appears to have carried over as the group of management and marketing scholars from institutions located in the western United States continued to publish together.

In addition a number of new smaller components emerged, one of which actually appears to have been a sort of break from the larger component of Canadian scholars. The separated group is comprised of several individuals who self-identified themselves as qualitative researchers in their responses to the questionnaire and who are connected via one focal scholar who had appeared in the larger Canadian component in the previous time block. Several of the scholars connected to the focal scholar identified the focal scholar as their doctoral advisor indicating a position as students of a structural "star" in the network at that time.

Some themes describing the remainder of the components include: 1) several isolates and smaller components comprised of individuals with physical education 
backgrounds; 2) three components of individuals from institutions outside of the United States and Canada; and 3) two components comprised of several scholars in the United States with a background in business.

From 1990-1994, it appears that not only was there a continued growth in the number of distinct connected groups, the number of actors in a few of the connected groups also appear to be much greater in volume. In fact, several components clearly stand out in the image as a result of the number of actors they include. Interestingly, some of the actors in the component from the previous time blocks consisting of marketing and management scholars in the United States became connected with another group of business scholars in the United States that had been present in the previous time block via a single intermediary actor.

Likewise, a few actors from the Canadian component in the first time block became connected to several actors from other components in the previous time blocks from the United States with a strong physical education background. It is likely the actors became affiliated because two of the focal actors from the two distinct groups from the prior time blocks became affiliated with Institution “Q.” While the two actors did not actually collaborate together, they became connected via intermediary actors who identified themselves as the two focal actors' advisees at Institution "Q." As such, there appeared to be a mixing of actors from both the United States and Canada.

While the previous two descriptions apply to two of the larger components that appear in the time block from 1990-1994, two of the other larger components also manifest some compelling themes. One of the components is comprised of a group of actors with primarily physical education backgrounds affiliated with one institution in a 
Midwestern state in the U.S. The second of the remaining large components illustrates collaboration between the focal actor with a qualitative focus from Canada who had seemingly broken off from the larger component in the earliest time block and a focal actor from one of the components of business scholars from the United States. Further exploration indicated that the two actors in that time block were both affiliated with Institution "A" in Canada.

Finally, a fifth component in the network for the time block from 1990-1994 was characterized by some very intense ties between three actors, one of which was joined to two other subgroups of actors. The intense triadic relationship between the three actors appears to be related to the fact that all three were scholars at Institution "L." Moreover, all three specialized in a similar area.

In the time block from 1985-1999, several relatively large components appear in the global network structure. One of the components, located in the bottom left-hand corner of Figure 4.6 clearly appears to have a "star-like" presence with one focal actor collaborating with a great number of other actors who in turn have very few ties with one another. This particular component is comprised of scholars from institutions located in the southwest part of the United States with backgrounds in such fields as psychology, exercise physiology, physical education, business, and communications. The focal actor or structural "star" appears to have a very interdisciplinary background with noted specializations in research design and statistics as well as a long history of significant acquisition of grant money.

The relatively large component located slightly higher and to the right in Figure 4.6 is characterized by several highly connected subgroups and one actor serving as an 
intermediary connecting both the subgroups. One theme that can be surmised about this component is the fact that the scholars all appear to either be students or institutional colleagues of the bridging actor with specializations primarily in physical education and sport sociology. Moreover, while there is considerable overlap between the institutional affiliations of the actors within the component, there is very little overlap between the actors in the component and the rest of the actors in the global network.

The large component located near the center of Figure 4.6 contains some very interesting themes. While comprised primarily of actors with considerable overlaps in institutional affiliations, namely Institutions “J,” “Q,” "L,” and “M,” which are all located in the United States, there are also a few actors singly tied to authors with Canadian institutional affiliations. While the Canadian affiliates themselves are not highly connected, they are connected via intermediary ties through actors affiliated with Institution “Q.” Incidentally, this time block was characterized by a period of a relatively high amount of actors moving from one institution to another, but mainly between Institutions “J,” "Q," "L," and "M.” Many of the institutional changes stemming from "Q" and going to others can be surmised as being doctoral graduates transitioning to professorial roles, while the overlaps between Institutions “J," "L," and "M" appear to be actors leaving one professorial position for another professorial position.

Located slightly to the right of the above described component in Figure 4.6 is another relatively large component with some intriguing characteristics. This component is comprised of actors with predominantly Canadian institutional affiliations and actors who primarily utilize qualitative research methods. In addition, the component appears to consist of some distinct structural features; particularly two actors with "star-like" 
characteristics connected by mutual intermediaries. The two "stars" and the intermediaries were all actors found in the previous time blocks in components with similar features (i.e., predominantly Canadian institutional affiliations and qualitative methods).

While the time block from 1995-1999 possessed a number of other intriguing components that would be well-suited for further investigation, time and space do not permit a further analysis here. However, a couple of general descriptions can easily be reported. First a number of components exist containing four or five actors all connected to each other but with minimal intensities (i.e., published together only once). Although not all of the structural components can be conclusively determined to fall into this category, a general theme suggests that these are actors who all published together one time on a single paper. Secondly, several other relatively large components of the network were not described in depth for two main reasons: 1) two of the components were comprised of actors primarily from institutions from outside the United States and Canada, and 2) the components were comprised of actors who had not served as participants and only published one or two times so attribute information for these actors was particularly sparse.

Incidentally, the time period from 2000-2004 provides us with the first global network structure in which we begin to see one relatively large component present in the image. We can surmise that within that large component, a number of the actors had coauthored with each other on multiple papers. Likewise, although the time block for 2005-In Press represents only about half of the time blocked for the other periods, we can 
make out some similar patterns. That is, there appears to be one much larger component of actors with varying strengths in ties penetrating the global structure.

As the complexity of the larger components makes it difficult to interpret the intricacies of their structural features, it is useful instead to first explore those components that are separate from the main components. In 2000-2004, several rather distinct connected components are present in addition to the main component. One of the components is very similar, and in fact contains many of the same actors, as the component from the previous time block of authors with specializations in physical education and sport sociology. Once again while there appears to be a great deal of overlap between the institutional affiliations of those within the component, there is seemingly very little institutional overlap between the actors in the component and the rest of the actors in the global network structure.

A second relatively large component in the time block from 2000-2004 consists primarily of one focal actor serving as a bridge (i.e., intermediary) between several somewhat cohesive subgroups. A general observation suggests that the actor's connections to the subgroups stem from his/her relationship as a doctoral advisor to one or more of the subgroup members. In addition, the focal actor possesses a specialization in sport sociology as do a number of the authors to which he/she is directly tied.

Two other components in the time block from 2000-2004 are comprised of one focal actor with solely Canadian institutional affiliations, a number of ties to some other actors with predominantly Canadian institutional affiliations, and a few actors with institutional affiliations outside of the United States and Canada. A third relatively large 
component is comprised of one actor with affiliations to institutions in Canada as well as an institution outside of Canada and the United States who serves as a bridge between a few fairly connected subgroups.

For the time block from 2005-2007, one of the larger components separate from the largest component consists of eight primary actors affiliated solely with Canadian institutions who consistently appeared throughout the various time blocks. Though it is not a highly connected component overall, it is the ties from the seven actors serving as intermediaries to one another and all of the other actors in the component that constitute the connectivity of the component. Interestingly, all eight of the actors identified themselves as predominantly qualitative researchers. In addition, although the advisor is not actually a focal person in the component, four of the more central actors in the component were, at some point in time, the actor's advisees.

In general, there appears to be significant overlaps between some of the actors in components in 2000-2004 and the actors in components 2005-In Press. For example, the two focal actors from separate components with only Canadian institutional affiliations from the time block 2000-2004 appear together in the same component for the time block from 2005-2007. Likewise, one relatively connected component exists comprised only of actors who are affiliated with institutions outside of Canada and the United States.

Although conceptually these pictures with the presence of one much larger component offer some insights into how the actors are tied to one another within the overall network, it can be very difficult to interpret the network beyond these initial observations particularly for some of the larger and more complex structures. Moreover, it is often hard to draw conclusions and make sense of a network in general simply 
through an initial visual inspection. Thus, social network analysis also allows many of the structural features of a network to be quantified.

Some quantified network measurements provide information about a single actor's relationship to the overall structure (ego-network measures), others look at more localized parts of the network (partial-network measures), and still others characterize the network as a whole (global network measures). Exploring the network through these measures allows researchers to be much more concise as well as precise in their interpretations. Nonetheless, the outputs of these quantified measurements can at times also be unwieldy and somewhat abstract. Therefore, combining the visual graphs with the calculated network indices provide the best conceptual understanding of a network's patterns.

\section{Quantification}

A report on the global network structure of sport management coauthorship for all of the time blocks combined specified that the network of 1,494 actors possessed 3,745 relations between actors with 1,306 of those ties being multiple relations (i.e., the authors produced multiple publications with one another). Table 4.3 provides the frequency distribution of the intensity (multiplicity in terms of the number of times two actors published together) of all lines in the network. The minimum intensity of any line is one meaning that the relationship between two authors is such that they have published together one time. According to the distribution, about $79 \%$ of all relationships in the network possess tie strengths of one. The maximum intensity of any line was 39 which, 
according to the distribution, occurred one time (i.e. one pair of authors published together 39 times).

\begin{tabular}{lrrrrrrr}
\hline $\begin{array}{c}\text { Tie } \\
\text { Strength }\end{array}$ & \multicolumn{1}{c}{ Total } & Pre-1985 & \multicolumn{1}{c}{$1985-$} & \multicolumn{1}{c}{$1990-$} & \multicolumn{1}{c}{$1995-$} & $2000-$ & \multicolumn{1}{c}{ 2005-In } \\
\hline 1 & $78.66 \%$ & $85.37 \%$ & $85.21 \%$ & $84.86 \%$ & $82.87 \%$ & $77.89 \%$ & $84.54 \%$ \\
2 & 11.37 & 7.32 & 9.86 & 8.10 & 11.01 & 13.10 & 10.99 \\
3 & 3.86 & 2.44 & 1.41 & 2.46 & 2.29 & 5.31 & 2.23 \\
4 & 2.59 & 3.66 & 2.11 & 1.41 & 0.92 & 1.42 & 1.68 \\
5 & 0.94 & 0.00 & 0.00 & 1.06 & 1.68 & 0.85 & 0.19 \\
6 & 0.78 & 0.00 & 0.00 & 0.35 & 0.46 & 0.57 & 0.19 \\
7 & 0.53 & 1.22 & 1.41 & 0.35 & 0.31 & 0.28 & 0.00 \\
8 & 0.04 & 0.00 & 0.00 & 0.00 & 0.00 & 0.00 & 0.00 \\
9 & 0.21 & 0.00 & 0.00 & 0.00 & 0.00 & 0.19 & 0.00 \\
10 & 0.29 & 0.00 & 0.00 & 0.00 & 0.00 & 0.09 & 0.00 \\
$>10$ & 0.72 & 0.00 & 0.00 & 1.06 & 0.45 & 0.27 & 0.19 \\
$\mathrm{~N}$ & 2439 & 82 & 142 & 284 & 654 & 1054 & 537 \\
\hline
\end{tabular}

Table 4.3 Frequency Distribution of the Intensity of Lines in the Global Network

\section{Connectivity}

One way to go about understanding the patterns of connectivity in the network is to look at structural attributes pertaining to the actors in the network. For example, it is useful to have a general grasp on the distribution of vertex degrees in the network. The degree of a vertex is the number of lines it has tying it to other vertices. As such, vertices with high degrees are often found in dense sections of the network because these vertices possess more ties (de Nooy et al., 2005).

To look at the degree distributions in a coauthorship network we can calculate the degree values for unique ties (whether or not one author has ever coauthored with another author). The degree value for a vertex provides an indication of the number of different people with whom that actor has coauthored. To calculate the average degree of all 
vertices for the network, Pajek was used to create a partition (categorization of vertex attributes into clusters) which was then used to generate a vector (i.e., where each vertex is then assigned a value based upon the cluster to which it belongs).

The average degree for all vertices in the total network for all time blocks was 3.26 with a standard deviation of 5.07. In other words, the average number of ties each vertex holds is about 3.3. The minimum vertex degree value was zero (i.e., actor(s) not observed to have coauthored with anyone else in the system), and the maximum vertex degree was 83 (i.e., actor observed to have coauthored with 83 other people). Table 4.4 provides the frequency distributions of degree values for the actors for each designated time block and ultimately represents the distribution of unique collaborators. Once again, the columns in the distribution represent the unique coauthorship patterns for each time block and the total column combines the carry over of unique ties and strength into a single measurement. That is, the total column represents the degree values for the actors over all of the time periods combined. 


\begin{tabular}{lccccccc}
\hline Count & Total & Pre-1985 & $1985-$ & $1990-$ & $1995-$ & $2000-$ & $\begin{array}{c}2005- \\
\text { In Press }\end{array}$ \\
\hline 0 & $8.30 \%$ & $0.00 \%$ & $15.49 \%$ & $19.24 \%$ & $10.83 \%$ & $8.02 \%$ & $5.66 \%$ \\
1 & 27.64 & 32.26 & 30.99 & 34.36 & 32.49 & 28.57 & 26.10 \\
2 & 25.84 & 29.03 & 28.17 & 36.12 & 26.17 & 23.18 & 21.70 \\
3 & 12.25 & 20.97 & 11.27 & 6.53 & 9.93 & 12.97 & 16.04 \\
4 & 8.23 & 9.68 & 8.45 & 5.50 & 6.32 & 9.46 & 8.18 \\
5 & 5.76 & 3.23 & 2.82 & 1.72 & 9.03 & 4.81 & 8.81 \\
6 & 2.48 & 1.61 & 0.00 & 1.03 & 0.90 & 2.04 & 1.89 \\
7 & 1.61 & 0.00 & 0.70 & 0.34 & 1.26 & 2.62 & 1.89 \\
8 & 1.67 & 0.00 & 0.00 & 0.34 & 0.72 & 3.06 & 1.57 \\
9 & 1.67 & 0.00 & 0.70 & 3.44 & 0.72 & 1.02 & 2.83 \\
10 & 0.54 & 0.00 & 0.00 & 0.69 & 0.54 & 0.87 & 0.00 \\
11 & 0.33 & 0.00 & 0.00 & 0.00 & 0.00 & 0.29 & 0.63 \\
12 & 0.40 & 1.61 & 0.70 & 0.00 & 0.36 & 0.58 & 0.94 \\
$>12$ & 3.38 & 1.61 & 0.70 & 0.69 & 0.72 & 2.48 & 3.77 \\
$\mathrm{~N}$ & 1494 & 62 & 142 & 291 & 554 & 686 & 318 \\
\hline
\end{tabular}

Table 4.4 Distributions of Unique Collaborators per Author for Each Time Block

As actors who are represented by a degree value of one or higher can be understood to be connected to at least one other actor, those who have a degree value of zero can be construed as isolates in the network. Incidentally, it is important to realize that although many individuals may be connected to many others, the actors in the system may not necessarily be tied together into one completely connected group. That is, sometimes a network can actually be a collection of groupings of individuals (Figure 4.3 provides a visual image of this sort of phenomenon). Therefore, it is also valuable to know about connectivity properties beyond the number of ties each actor possesses.

To further explore the connectivity properties of the network, we can look beyond the information about individual relations and the number of ties each actor possesses and analyze how the ties come together to form the entire network. One such property is 
called density. In essence, the density of a network refers to the percentage of lines present in the network relative to the total possible number of lines that could be present given the number of actors in the system.

An analysis of the global network for all time periods found the density for the overall network to be .002 which fundamentally means that only .2 percent of all possible ties among actors in the system are present. Obtaining such a low density value for a network of this size is not unusual because density tends to be inversely related to network size. That is, the larger the network, the lower the density tends to be (de Nooy et al., 2005). Intuitively, this makes sense because as the number of actors in a system increases, the number of potential ties also rapidly increases. However, in all reality, the number of ties a person can maintain with others is limited (de Nooy et al., 2005; Scott, 2000).

As density is so dependent on the size of the network thus making it difficult to compare two networks of different sizes, in many cases, knowing the density of the overall network is not particularly useful (de Nooy et al., 2005). Therefore, it is necessary to explore other measures to obtain a better indication of global network connectivity. One such measure is termed reachability. Assessing the reachabilty of a network explores the extent to which it is possible to reach one node from another node and serves as a more effective indicator of network connectivity than density.

To conceptually understand what the reachability of a network holds for us, it is useful to envision the nodes of the network as being people who possess information and ties between the nodes as potential pipelines for the transmission of information from one person to another. Whether or not the information reaches a certain person usually 
necessitates that the person have access into the pipeline of ties in which the information is circulating. While it is possible for the information to pass through intermediaries, if there is not a pipeline of ties between two actors, structurally it is not possible for the message to travel from one actor to the other.

One of the most general ways of assessing the patterns of connectivity from this standpoint is through a form of connection called a walk. As the word "walk" might imply, this property involves the ability to travel from one vertex to another through the lines connecting the actors in the system (i.e., the presence of a connectivity pattern that allows you to get from one vertex to another). Thus, in essence, a walk is a sequence of actors and relations that begins and ends with actors (Hanneman \& Riddle, 2005). By definition, walks can be relatively unrestricted such that a single "walk" can involve the same actor or same relation any number of times.

In some ways, simply using the presence of walks can be problematic because routes can be circular within a single group of actors and not all that indicative of the cohesiveness of the structure. Therefore, scholars often articulate that a more useful property for assessing connectivity patterns in the system is the presence of a path. A path is simply a walk of any length in which no vertex between the first and last vertex of the walk occurs more than once (i.e., you do not use a vertex or relation more than once to get to another vertex) (de Nooy et al., 2005). Under these premises, a network is said to be connected to the extent that we can "walk" from each vertex to all other vertices (without reusing a node or tie).

Substantively, knowing the paths in a global network is important to understand because the inventory of the total connections among all of the actors provides the 
researcher with a sense of the closeness of each pair of nodes as well as a feeling for how closely coupled the entire system is overall (Hanneman \& Riddle, 2005). Thus, the quantified differences in paths between actors theoretically depicts the potential for information (and in many cases influence) exchange in the network. In other words, this information can be used to discern which actors (i.e., authors) in the system are likely to be (or to have had) direct influences on each other by means of collaboration on at least one publication.

From the initial drawing shown in Figure 4.1, we already know that our total global network is not connected by the "path" standards as it contains a number of isolates. Therefore, in order to further investigate the connectivity patterns of the network, it is helpful to extract various parts of the network for closer inspection. One way social network analysis allows us to detect information on parts of the network is to extract what are called partitions. A partition of a network is essentially a subset or cluster of the network's vertices and can be very useful for extracting parts of the network to reduce its size and complexity (we did this earlier to aid in the visualization process and to obtain the vertex degree values).

Partitioning the network is particularly useful to attempt to find one of the most intriguing and, in many cases, valuable properties of networks - the presence of cohesive sub-groups. Substantively, the presence of cohesive subgroups in a network is often used to make the argument that people's social relations often tie them into circles or subnetworks of actors in which unique norms, values and perspectives develop. At times, the cultural distinctions of these subgroups may run counter to cultures of other subgroups and even to a mainstream or dominant culture (Scott, 2000). Tying this 
argument back into what subgroups of actors in communities of scientific practice might hold for the idea space of a particular field, the presence of distinct subgroups of actors could relate to the paradigms that they embrace and the institutional ways in which they go about generating knowledge.

The starting point for measures assessing the subgroup structures of a network is through the extraction of sub-graphs. A sub-graph is essentially any collection of points selected from the whole graph with the ties between the points remaining in tact. A useful approach to understanding the sub-graphs of a network is to define some meaningful category of attributes for the actors in the global network (e.g., actors connected by certain path standards or institutional affiliation) and then explore the resulting subgraphs for patterns.

Under these premises, we can manipulate the network in a couple of different ways. First, we can reduce the network in size by removing all of the isolates (i.e., nodes with a degree value of 0). Secondly, we can partition the coauthorship network obtained by identifying what are referred to as components in the network and examine the resulting subgraphs. A component of a network consists of all vertices that are tied to each other by at least one path. We partitioned the network into components earlier to help with the visualization of the time blocks.

It is important to note that by removing all of the vertices with a degree value of zero, we reduce the number of actors in our network to 1,370 but still maintain the total number of lines $(3,745)$ and multiple lines $(1,306)$. In looking at the report on the cluster distribution of the components' partition, we can tell that once the isolates are removed, the network can be broken down into 143 components of a minimum size of two vertices. 
In doing so, we discover that the largest component is composed of 927 vertices and the second largest component is comprised of 26 vertices. As such, we can surmise that about $62 \%$ (927 authors) of the actors in our overall network (with isolates included) for all blocks of time are contained within the largest component in the network.

Conceptually, these indices show that the observed global network contained 124 authors who never co-authored with anyone else in the system and one main component containing 927 authors who are at least minimally connected by path standards. In addition, there are 142 other components within the network ranging in size from two vertices to 26 vertices. With this information in mind, we can begin to break down the concentration of ties (or connectivity) within the components.

In addition to whether or not we can reach one node from another, it is also useful to consider how many ways in which it can be done. That is to say, once we establish whether two actors have a pipeline for a message to travel between them, we can then look at whether or not it is possible to reach them in multiple ways (i.e., different paths for the message to travel from one person to another). Intuitively, the presence of multiple paths is an important thing to consider because it can indicate the extent to which information can still reach actors in the event a single actor leaves as well as project the potential for a message to matriculate to an individual multiple times.

As an initial exploration into this idea, we can extract what is called a bicomponent. Fundamentally, the bicomponents of a network are nested within its components and require that there be at least two-independent paths connecting every pair of actors. In other words, a bicomponent portrays the skeleton of the network that is left after all of the nodes which are connected by the network by only one path are 
removed. In addition, this conception can be extended to look at tricomponents, 4components and beyond to identify increasingly cohesive (connected) subgraphs (Moody and White, 2003). Table 4.5 provides information about the components for each time block and Table 4.6 provides information about the bicomponents for each time block.

\begin{tabular}{|c|c|c|c|c|c|c|c|}
\hline & Total & $\begin{array}{l}\text { Pre- } \\
1985\end{array}$ & $\begin{array}{l}1985- \\
1989\end{array}$ & $\begin{array}{l}1990- \\
1994\end{array}$ & $\begin{array}{l}1995- \\
1999\end{array}$ & $\begin{array}{l}2000- \\
2004\end{array}$ & $\begin{array}{l}2005-\text { In } \\
\text { Press }\end{array}$ \\
\hline $\begin{array}{l}\text { Largest } \\
\text { Component }\end{array}$ & & & & & & & \\
\hline $\begin{array}{l}\text { Size } \\
\text { Second }\end{array}$ & 927 & 18 & 19 & 26 & 73 & 365 & 136 \\
\hline $\begin{array}{l}\text { Largest } \\
\text { Component } \\
\text { \# of } \\
\text { Comps. } \\
\text { Size: }\end{array}$ & 26 & 16 & 13 & 22 & 38 & 17 & 21 \\
\hline 2 & 75 & 1 & 12 & 28 & 51 & 45 & 22 \\
\hline 3 & 41 & 1 & 3 & 12 & 23 & 19 & 10 \\
\hline 4 & 12 & 1 & 1 & 2 & 8 & 5 & 1 \\
\hline 5 & 5 & 0 & 3 & 4 & 4 & 0 & 2 \\
\hline 6 & 4 & 2 & 3 & 3 & 3 & 3 & 1 \\
\hline$>6$ & 6 & 3 & 4 & 7 & 13 & 8 & 6 \\
\hline Total & 143 & 8 & 26 & 56 & 102 & 80 & 42 \\
\hline
\end{tabular}

Table 4.5 Distribution of Components for Each Time Block 


\begin{tabular}{|c|c|c|c|c|c|c|c|}
\hline & Total & $\begin{array}{l}\text { Pre- } \\
1985\end{array}$ & $\begin{array}{l}1985- \\
1989\end{array}$ & $\begin{array}{l}1990- \\
1994\end{array}$ & $\begin{array}{l}1995- \\
1999\end{array}$ & $\begin{array}{l}2000- \\
2004\end{array}$ & $\begin{array}{l}2005-I n \\
\text { Press }\end{array}$ \\
\hline \multicolumn{8}{|l|}{ Largest } \\
\hline \multicolumn{8}{|l|}{ Bi-Comp. } \\
\hline Size & 406 & 5 & 6 & 11 & 22 & 145 & 81 \\
\hline \multicolumn{8}{|l|}{ Second } \\
\hline \multicolumn{8}{|l|}{ Largest } \\
\hline Size & 16 & 4 & 5 & 7 & 17 & 13 & 10 \\
\hline \multirow{2}{*}{\multicolumn{8}{|c|}{ \# of }} \\
\hline & & & & & & & \\
\hline \multicolumn{8}{|l|}{ Size: } \\
\hline 3 & 119 & 9 & 15 & 25 & 49 & 52 & 19 \\
\hline 4 & 30 & 4 & 6 & 5 & 10 & 16 & 8 \\
\hline 5 & 9 & 1 & 2 & 4 & 6 & 7 & 4 \\
\hline 6 & 4 & 0 & 1 & 1 & 8 & 1 & 3 \\
\hline$>6$ & 8 & 0 & 0 & 2 & 6 & 8 & 4 \\
\hline Total & 170 & 14 & 24 & 37 & 79 & 84 & 38 \\
\hline
\end{tabular}

Table 4.6 Distribution of Bi-Components for each time block.

While breaking down the network by components (bicomponents, tricomponents, etc.) can provide us with an understanding of the overall path connectivity of the network, we can also look at connectivity and the presence of subgroups from some different angles. Although we already know the distribution of degree values for the vertices and thus have a basic knowledge of the concentration of ties around the individual vertices, we do not know whether the vertices with high degree values are clustered together or scattered all over the network (i.e., amongst the components or even sub-groups of the components).

One measure that can help bridge this gap between the concentration of ties on a single node and the connectivity within the components is the assessment of $k$-cores. By definition, a $k$-core is a maximal subgraph in which each vertex has at least a degree value of $k$. For instance, a 2-core would consist of all vertices that are connected to at 
least two other vertices within the core. The "maximal" stipulation infers that we are interested in extracting the largest set of vertices that satisfy the desired $k$ value (de Nooy et al., 2005).

It is useful to imagine the k-cores as being stacked on top of one another such that all of the vertices in the 4-core are included in each of the cores below, but not all of the vertices in the cores below are included in the 4-core. Only those vertices with a minimum degree of 4 are included in the 4-core. In other words, a vertex may belong to more than one core simultaneously, and the cores are nested such that higher $k$-cores are always contained within the lower $k$-cores. Fundamentally, $k$-cores are useful in helping to identify relatively dense subnetworks which can then be used to find some of the larger cohesive (i.e., highly connected) subgroups in a network. That being said, it is important to recognize that a $k$-core is not necessarily a cohesive subgroup (in essence a subset of actors among whom the density of the subgraph is relatively high) itself but rather an indicator of where we should explore further for highly concentrated groups of actors (de Nooy et al., 2005).

Using Pajek, a partition of the cores for the largest component in the total network (with all time blocks combined and multiple relations summed into a single tie) identified 9 cores. Figure 4.9 provides a color/grayscale illustration of the network with the isolates and all other components aside from the largest removed (i.e., only the largest component was assessed) whereby the color and number by the node are indicative of the $k$-core to which it belongs. Although visually the picture does not provide much insight initially, a closer inspection reveals a number of areas of subnetworks with vertices of high degree concentrations. Table 4.7 provides the corresponding frequency distribution for all of the 
time periods combined and for the time blocks of 2000-2004 and 2005-In Press. The rest of the time periods were excluded because the components in previous years were relatively small and reasonably interpreted through visualization. The frequency distribution below indicates that a great majority of the networks are comprised of vertices belonging to the 5-core or below.

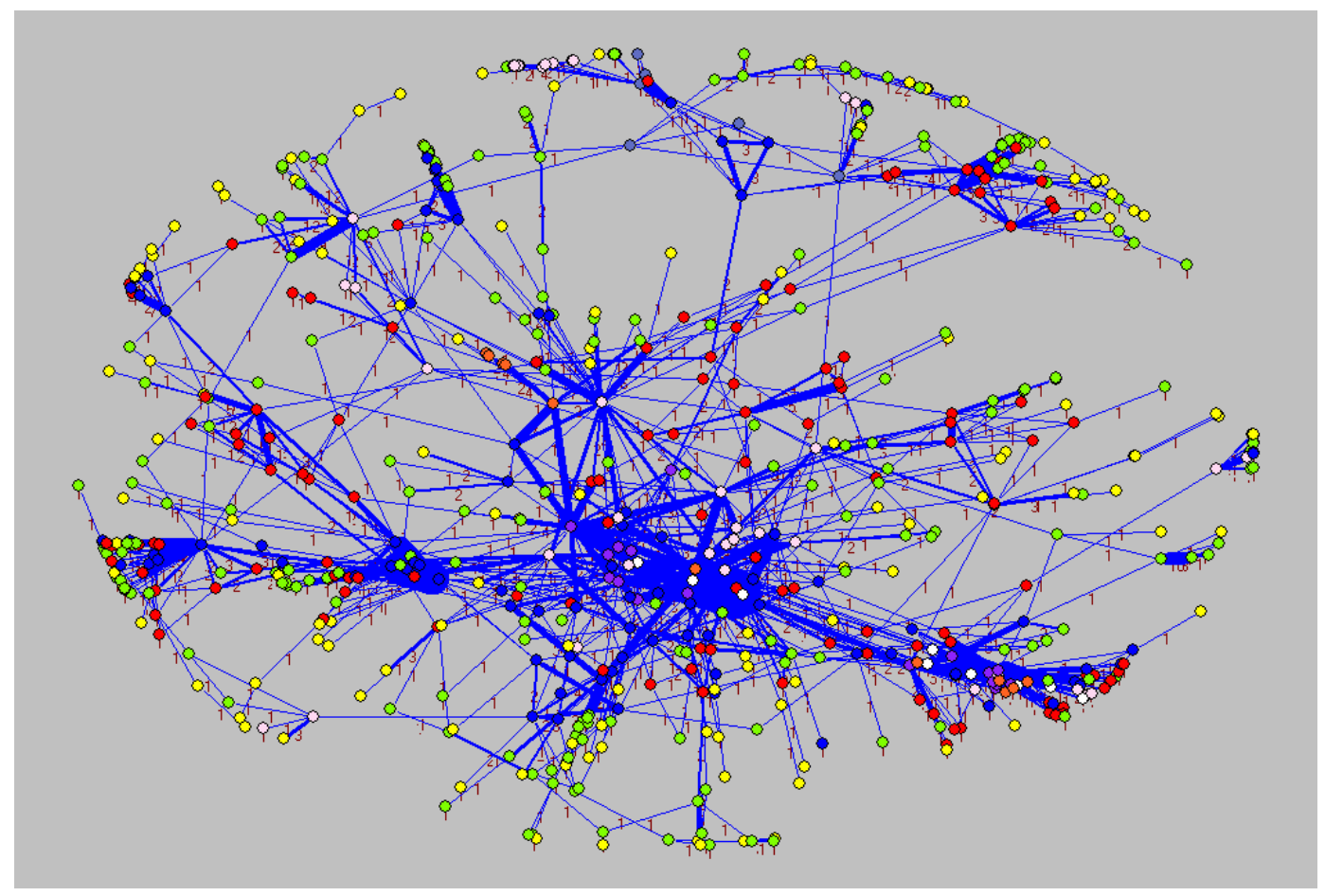

Figure 4.9 Illustration of the $K$-core Groups for the Main-Component 


\begin{tabular}{|c|c|c|c|c|c|c|c|c|}
\hline \multicolumn{3}{|c|}{$\begin{array}{l}\text { Largest Component } \\
\text { All Time Periods }\end{array}$} & \multicolumn{3}{|c|}{$\begin{array}{c}\text { Largest Component } \\
2000-2004 \\
\end{array}$} & \multicolumn{3}{|c|}{$\begin{array}{c}\text { Largest Component } \\
2005-\text { In Press } \\
\end{array}$} \\
\hline $\begin{array}{l}\text { Highest } \\
\text { K-core }\end{array}$ & $\begin{array}{l}\text { Freq. of } \\
\text { Vertices }\end{array}$ & $\begin{array}{l}\text { Freq. } \\
\text { Percent }\end{array}$ & $\begin{array}{l}\text { Highest } \\
\text { K-core }\end{array}$ & $\begin{array}{l}\text { Freq. of } \\
\text { Vertices }\end{array}$ & $\begin{array}{l}\text { Freq. } \\
\text { Percent }\end{array}$ & $\begin{array}{l}\text { Highest } \\
\text { K-core }\end{array}$ & $\begin{array}{l}\text { Freq. of } \\
\text { Vertices }\end{array}$ & $\begin{array}{l}\text { Freq. } \\
\text { Percent }\end{array}$ \\
\hline 1 & 235 & $25.35 \%$ & 1 & 90 & $24.66 \%$ & 1 & 17 & $12.50 \%$ \\
\hline 2 & 277 & 29.88 & 2 & 105 & 28.77 & 2 & 37 & 27.21 \\
\hline 3 & 164 & 17.69 & 3 & 74 & 20.27 & 3 & 43 & 31.62 \\
\hline 4 & 118 & 12.73 & 4 & 51 & 13.97 & 4 & 18 & 13.24 \\
\hline 5 & 67 & 7.23 & 5 & 14 & 3.84 & 5 & 12 & 8.82 \\
\hline 6 & 13 & 1.40 & 6 & 9 & 2.47 & 6 & 0 & 0.00 \\
\hline 7 & 15 & 1.62 & 7 & 13 & 3.56 & 7 & 0 & 0.00 \\
\hline 8 & 18 & 1.94 & 8 & 9 & 2.47 & 8 & 9 & 6.12 \\
\hline 9 & 20 & 2.16 & & & & & & \\
\hline
\end{tabular}

Table 4.7 Frequency Distribution of all K-cores All, 2000-2004, and 2005-2007

In wanting to further investigate the properties that the subgraphs possess as obtained through the $k$-core analysis, it is useful to have a stricter definition of what a cohesive subgroup entails. De Nooy et al. (2005) suggest one way to do through the concept of cliques. Cliques are a set of vertices in which each vertex is directly connected to all other vertices, or in other words, a subnetwork possessing maximum density. The number of vertices in a clique characterizes its size. While it is of course possible to have maximally connected subnetworks possessing only two connected nodes, in all reality, these are not particularly interesting in terms of cohesion because they represent only a dyad. Therefore, another condition for clique identification is such that a clique is a maximally connected subnetwork containing three or more vertices.

According to de Nooy et al. (2005), in social network analysis it is the structures from overlapping cliques which are regarded as cohesive subgroups noting: “Cliques or 
complete triads are the densest sections or 'bones' of a network, so the structure of overlapping cliques are considered its 'skeleton'”(p. 74). In looking at the cliques of our network with all time periods combined, we are really only interested in the fact that two actors have ever coauthored together. Therefore, we first removed all multiple relations by summing them into a single tie. We are also only really interested in the one main component (consisting of 927 actors), so we created a new network composed of only that component and analyzed it looking for all maximally connected triads. In total, the 10, 848 triads were found in the main component. In addition, the analysis revealed that 238 vertices (of the 972) were not included in any triad cliques while one vertex belonged to 1,188 triad cliques.

From this information a subnetwork consisting of 689 vertices (all a member of at least one triadic clique) composing 12 components was revealed. The largest of the components still contained 622 vertices as reported by the frequency distribution of the components shown in Table 4.8. Figure 4.10 provides an illustration of this network with the components separated from each other. Table 4.8 also breaks down the same distribution for the time blocks 2000-2004 and 2005-In press as these were the only two time blocks in which there appeared to be one principal component in the network. 


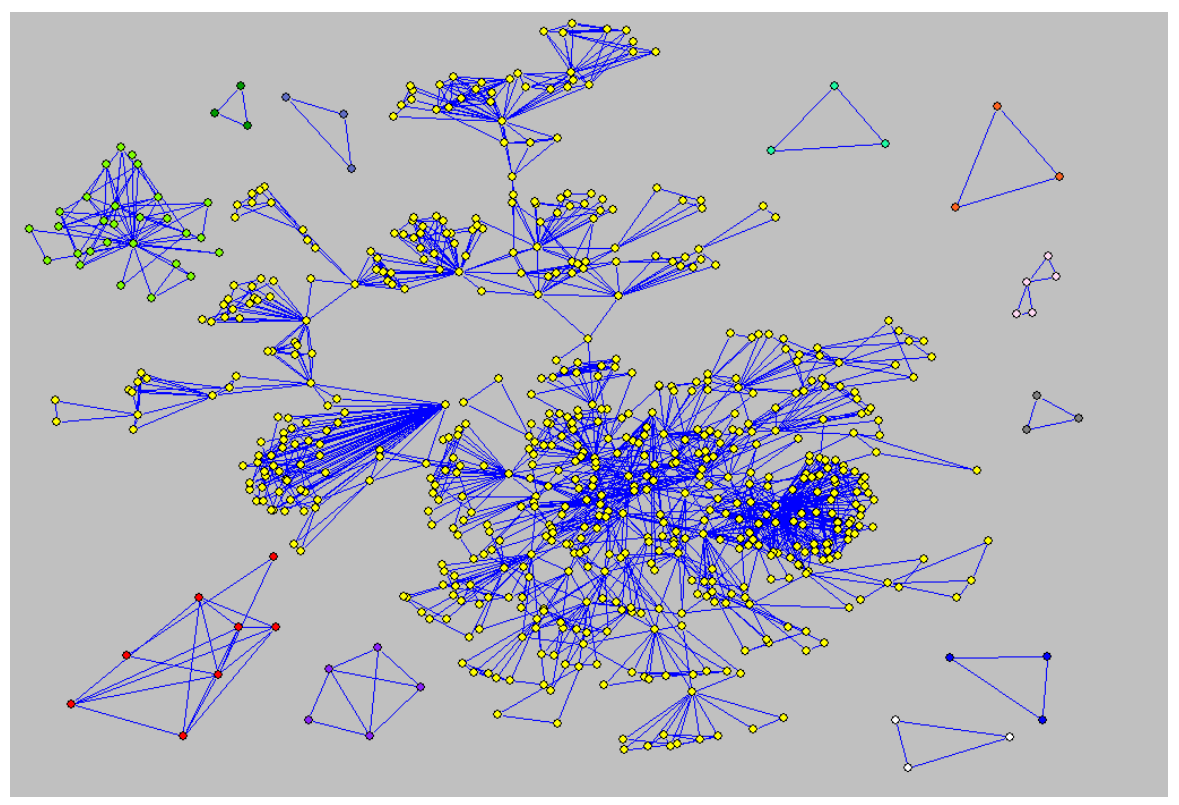

Figure 4.10 Subgraph of 3-Clique Overlaps of Main Component for all Time Blocks

\begin{tabular}{|c|c|c|c|c|c|c|c|c|}
\hline \multicolumn{3}{|c|}{ All Time Periods } & \multicolumn{3}{|c|}{$2000-2004$} & \multicolumn{3}{|c|}{ 2005-In Press } \\
\hline Comp. & $\begin{array}{l}\text { Freq. of } \\
\text { Vertices }\end{array}$ & $\begin{array}{l}\text { Freq. } \\
\text { Percent }\end{array}$ & Comp. & $\begin{array}{l}\text { Freq. of } \\
\text { Vertices }\end{array}$ & $\begin{array}{l}\text { Freq. } \\
\text { Percent }\end{array}$ & Comp. & $\begin{array}{l}\text { Freq. of } \\
\text { Vertices }\end{array}$ & $\begin{array}{l}\text { Freq. } \\
\text { Percent }\end{array}$ \\
\hline 1 & 622 & $90.28 \%$ & 1 & 45 & $16.48 \%$ & 1 & 4 & $1.90 \%$ \\
\hline 2 & 28 & 4.06 & 2 & 13 & 4.76 & 2 & 9 & 4.29 \\
\hline 3 & 8 & 1.16 & 3 & 15 & 5.49 & 3 & 3 & 1.43 \\
\hline 4 & 3 & 0.44 & 4 & 177 & 64.84 & 4 & 110 & 52.38 \\
\hline 5 & 5 & 0.73 & 5 & 3 & 1.10 & 5 & 16 & 7.62 \\
\hline 6 & 3 & 0.44 & 6 & 3 & 1.10 & 6 & 9 & 4.29 \\
\hline 7 & 3 & 0.44 & 7 & 3 & 1.10 & 7 & 9 & 4.29 \\
\hline 8 & 5 & 0.73 & 8 & 6 & 2.20 & 8 & 3 & 1.43 \\
\hline 9 & 3 & 0.44 & 9 & 5 & 1.83 & 9 & 6 & 2.38 \\
\hline 10 & 3 & 0.44 & 10 & 3 & 1.10 & 10 & 3 & 1.43 \\
\hline 11 & 3 & 0.44 & & & & 11 & 5 & 2.86 \\
\hline 12 & 3 & 2.16 & & & & 12 & 3 & 1.43 \\
\hline & & & & & & 13 & 3 & 1.43 \\
\hline & & & & & & 14 & 4 & 1.90 \\
\hline & & & & & & 15 & 3 & 1.43 \\
\hline & & & & & & 16 & 3 & 1.43 \\
\hline & & & & & & 17 & 6 & 2.86 \\
\hline & & & & & & 18 & 5 & 2.38 \\
\hline & & & & & & 19 & 3 & 1.43 \\
\hline & & & & & & 20 & 3 & 1.43 \\
\hline
\end{tabular}

Table 4.8 Distribution of Components after 3-Clique Assessment 
While clique assessment can help us identify groups of highly connected actors, there are some important considerations to keep in mind. According to Wasserman and Faust (1994), a clique is a very strict definition of cohesive subgroups since the absence of a single tie among subgroup members can prevent the group from meeting the clique definition. Moreover, Wasserman and Faust noted that using cliques to identify subgroups allows for no internal differentiation. That is, according to graph theoretic principle members are structurally identical. As such, it is difficult to determine whether there are some core actors who are more strongly connected to the subgroup than perhaps a few peripheral actors.

While not alleviating the problem of the strict nature of cliques, one way to focus on the differentiation of the internal structure of subgroups within a network is to study how the cliques overlap in the larger structure. We can get a general sense of the overlap of cliques by reassessing the $k$-cores after the cliques have been extracted and then partition out components layer-by-layer.

Fundamentally, our clique assessment was of importance because it gives us a more precise grouping of vertices with high concentrations of ties to other vertices with high concentrations of ties. That is, we now have a smaller set of vertices to look at using the $k$-core measures. In essence, what this clique information gives us is a sense of to what extent an author's coauthors have coauthored with each other by eliminating those that are only connected to fewer than $k$ actors in the core. As such, we can see that within the main component of the global network, there are 12 subgroups of various sizes that can be considered connected based upon the notion of overlapping clique conditions. 
Moreover, one of these subgroups is still fairly large containing almost 90\% (622 out of 689 authors) of the remaining actors in the extracted network.

Therefore, it is useful to use a $k$-core assessment to look at the largest nested subgroups within the extracted network components. To take a look at the most connected subgroups within this component, we can eliminate the lower $k$-cores for a better visual picture and distinguish between the components in order to get a better sense of the presence of the largest maximally connected cohesive subgroups. Figure 4.11 illustrates the three components derived from $k$-cores 6-9 from the components of the 3clique analysis.

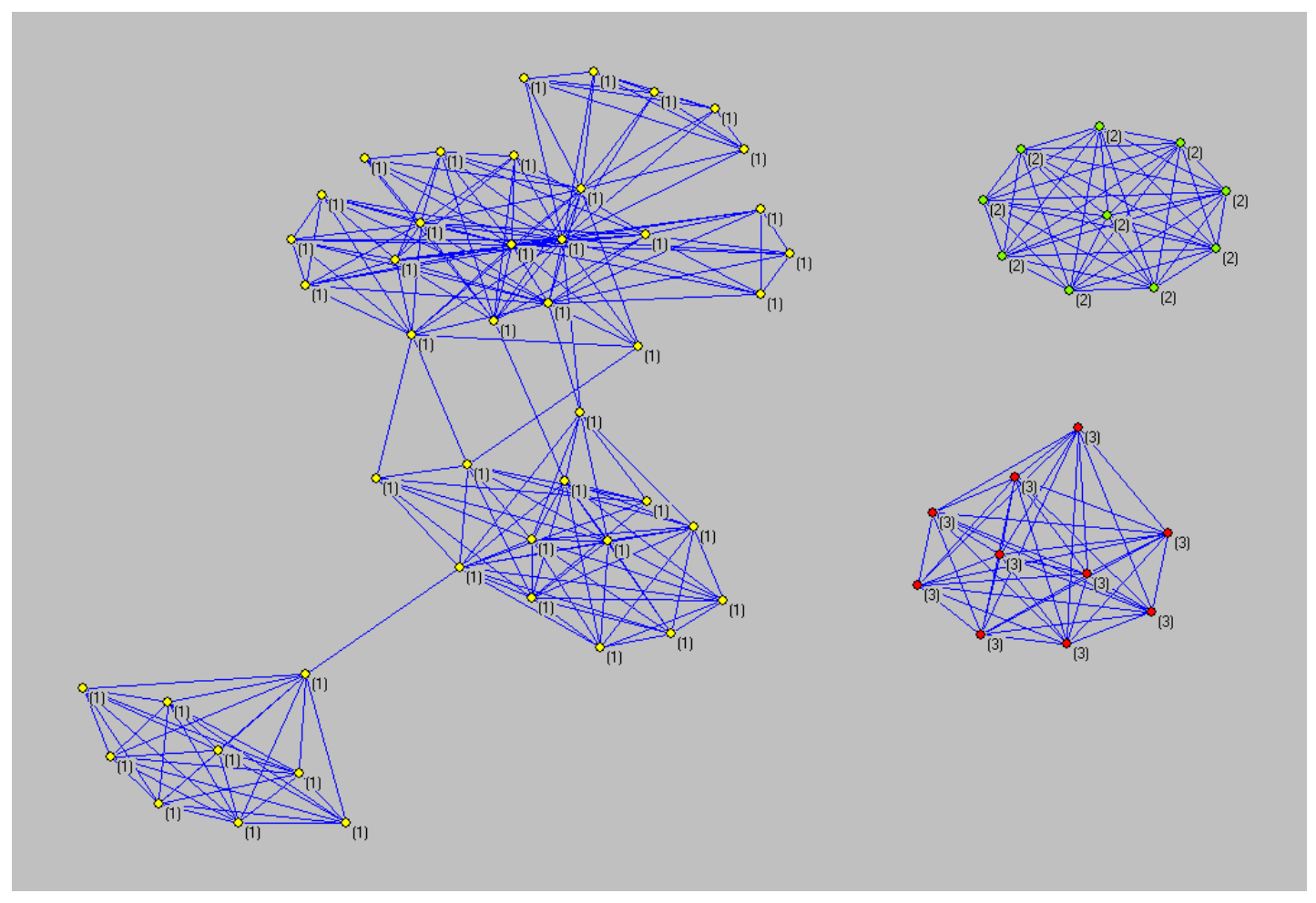

Figure $4.11 \mathrm{~K}$-cores 7-9 in the Derived Components from the 3-Clique Analysis 
To surmise what Figure 4.11 tells us, we now know that there are 66 authors in the system that form a fairly cohesive subnetwork within the main component (927 authors) of the global network (all 1494 authors). Within that subnetwork, a number of even more cohesive subgroups exist. In fact, even within the new largest component, we can make out several more subgroups that we could break out in which the actors only remain tied to each other because of a select few nodes.

Nonetheless, one of the limitations of these findings is that the reason a derived subgroup of coauthors is so highly connected may very well be that they simply collaborated on a single paper together. That is, some of the derived components may only be a reflection of the fact that a particular set of actors produced one publication as a group. This scenario is especially likely for the maximally connected components extracted where the density of the subgraph is equal to one.

One way to somewhat alleviate this problem is by looking at the intensity of ties between the authors. Factoring in the multiple relations between two vertices, Figure 4.12 illustrates the strength of ties between the authors within these subgroups. Once again, the thicker the line connecting the two vertices, the more often they have co-authored together. 


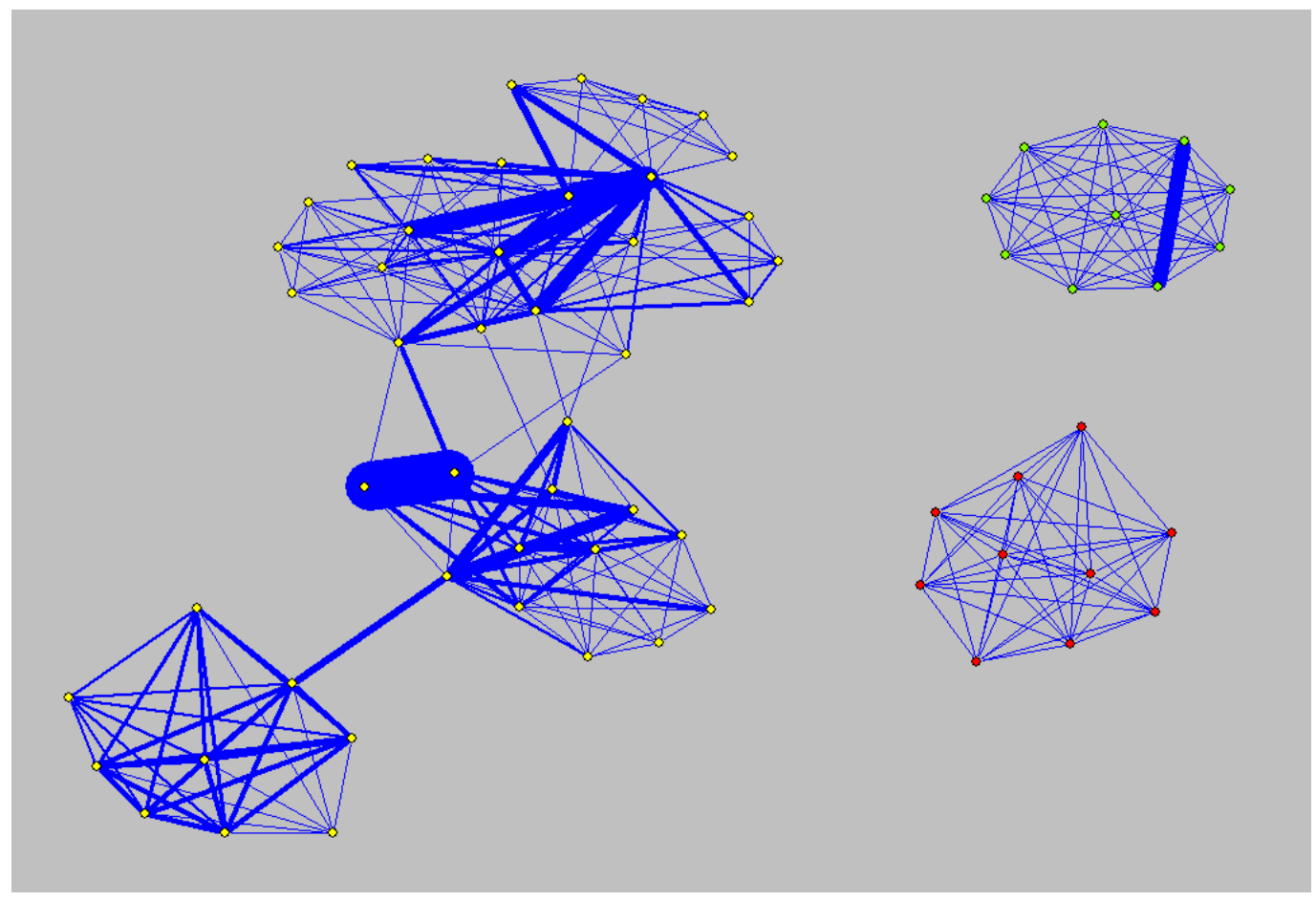

Figure 4.12 Intensity of Ties of K-cores 7-9 in the Components of the 3-Clique Analysis

A visual inspection of Figure 4.12 tells us that the components on the right-hand side of the image may indeed be the reflection of the fact that the authors in the subgroup all wrote a single paper together because their connectivity is so high and the intensities of the ties are so low (with the exception of one tie in the upper right-hand component). However, the fact that there are a number of stronger ties between actors in the largest of the remaining components in addition to a high level of connectivity indicates that the much of the connectivity of this subgroup is not entirely due to a single publication. Moreover, we can surmise that within the field of sport management, there is a relatively large, cohesive subgroup of actors, many of which have relatively strong relationships with one another. 
To see if any general themes can be derived based upon the attributes of the actors found in the three components of $k$-cores 7-9 extracted from the largest component after the clique assessment, the vertex identification numbers were compared to the subject attribute spreadsheet. Within the larger component, several themes were apparent. The subgroup within the largest component located in the bottom left-hand corner of Figure 4.12, is comprised of a group of scholars with specializations not really relevant to the field of sport management who produced a couple of publications together. Connecting this subgroup to the rest of the component is a single actor who possesses several specializations within the field of sport management and who graduated with a degree from Institution “Q."

The middle cohesive subgroup in the largest component in Figure 4.12 is comprised of a set of actors who are connected together through a number of publications and all but two of who received their doctoral degrees from Institution "Q." In fact many of the actors in this subgroup were advisees of the actor serving as the connecting point between the lower subgroup described in the previous paragraph. Aside from their affiliation with Institution “Q," the actors possess only very slight overlaps in terms of specializations and other institutional affiliations.

Two of the actors in the subgroup possess an extremely strong tie; one of who was a graduate of Institution "Q" and both of whom are affiliated with Institution "T." Both of these actors are tied to a third actor who is strongly connected to the third subgroup (located in the top left-hand area of Figure 4.12). This third actor also possesses an affiliation with Institution "T.” However, the actor also possesses an institutional 
affiliation with Institution "I" which as it turns out is a common affiliation among many of the actors in this third subgroup.

In addition, within the third subgroup, a number of actors possess institutional affiliations with either Institution "I” or Institution " $K$ ” and several possess affiliations with both. Interestingly, the authors possess a wide variety of specializations and are often tied together through the actor described earlier who has specializations in research design and statistics in addition to sport management related specializations. Incidentally, that particular actor possesses institutional affiliations with both Institution "I" and Institution "K."

Although the illustrations and distributions of the analyses are not provided here, the same procedures were carried out for the time blocks 2000-2004 and 2005-In Press to assess whether similar characteristics were present. The results indicated that in the time block from 2000-2004 the largest subgroup component was comprised of a relatively large, cohesive subgroup of actors who possessed varying strengths of relationships with one another; some of which were very intense (one triad and one dyad within the triad possess nearly twice the intensity of the next strongest ties in the network). Likewise, although the time block from 2005-In Press represents only part of a 5-year time block, it appears as if the presence of one dominant core of relatively cohesive, intense ties between actors will continue. Moreover, there appears to be a large overlap (i.e., many of the same exact actors) between the actors that appear in the largest cohesive component in the time block from 2000-2004 and the block for 2005-In Press.

Since the network for all time periods combined provides a more robust picture of the network and captures the overlap between blocks, it is not necessary to describe in 
great detail the structural themes that emerged predominantly during these time blocks. However, in order to emphasize two themes pertaining to the attributes of cohesive subgroups, it is important to highlight two general observations related to both time blocks. One of the observations emphasized the presence of a very strongly connected group of actors affiliated with Institution "Q” in both time blocks. The second observation is related to the divide between what appears to be actors with strong institutional affiliations to Canadian institutions and actors with strong institutional affiliations to institutions in the United States. While a select few actors appear to bridge the two groups with all periods combined, on the whole, there appears to be a very distinct structural gap between the two groups.

\section{Clustering}

Through the assessments of connectivity and cohesive subgroups, we have a general sense that several distinctive clusters of coauthors are present in the network. Although it is possible to quantitatively and visually break these clusters out for more precise interpretations, the measures and interpretation of the measures are outside the scope of this study. Instead, we can qualitatively generate several themes related to the clustering properties in the observed network. As such, the following paragraphs highlight the emergent themes.

Outside of the main component, a number of isolates and smaller clusters are comprised of many authors who only published once in the observed network. Some of these actors were comprised of practitioners and other were scholars from institutions outside the United States and Canada. In addition, a number of the clusters were 
comprised of scholars who had only published once or twice in the field of sport management but are likely to have published in an entirely different field of study. For example, several of the clusters were comprised of general management, marketing or economic scholars who happened to carry out a study related to sport.

There appears to be a large structural divide between many of the scholars who possess institutional affiliations only within the United States and scholars who possess institutional affiliations within Canada. While there are a couple of actors who act as brokers and ties between actors that act as bridges, even those ties appear to be relatively weak in intensity (i.e. the authors had only published together once or twice). Interestingly, the level of cohesion between the scholars from primarily Canadian institutions appears to be relatively low, while the level of cohesion between scholars with institutional affiliations in the United States ranges all the way from relatively low to very dense connectivity.

Within the main component, several relatively cohesive clusters of actors are present. One of the clusters is comprised of many scholars from institutions located in primarily the southeastern part of the United States. This cluster appears to be weakly characterized by the presence of a structural "star" who was self-identified as specializing in research methods and statistics. It seems reasonable to assume that one of the reasons for his/her "star-like" position is due to the fact that he/she bridges multiple specializations as a methodologist of sorts.

Also located within the main component, is a fairly dense cluster of actors all possessing institutional affiliations with Institution “Q.” Located within the cluster, two "star-like" actors are present who are not directly linked but whose coauthors are often 
linked to one another. The reasons for their designation as structural stars can likely be attributed to the fact that both have been in the field for more than 20 years, both are in the top $1 \%$ in terms of the number of publications they have produced, and both serve as brokers to other clusters of actors (i.e., both tied to different parts of the cluster of actors with only Canadian institutional affiliations). Finally, while a number of their coauthors were actually former students who ended up coauthoring with each other, each of them is also tied to a number of actors (some of which were also former students) who only appeared one time in the network and possessed no ties to any other actors in the network.

\section{Centrality}

Beyond understanding the presence of actors who possess a great number of ties and the extent of highly cohesive subgroups of actors, it is also useful to distinguish what the position of these actors holds substantively for the network of actors. On the whole, it is not uncommon for networks to contain actors (and/or subgroups of actors) who are located in positions that are structurally characterized as being more accessible to circulating information as well as more opportunistic in terms of spreading information. In addition, certain structural elements of a network determine how easily information can be diffused throughout the actors. Therefore, if we want to understand the structure of the network as a whole, we must first determine if there are actors who are structurally positioned in such advantageous ways and secondarily investigate whether or not there are certain attributes of those actors that have led to them being located in a particular position. 
To a certain extent, we have already analyzed the networks for the time blocks of prior to 1985, 1985-1989, and 1990-1994 because the components were small enough that visualization techniques allowed us to make reasonable interpretations of the structure. However, beginning in 1995-1999 and lasting through the time block for 2005In Press, some of the components were too large to meaningful interpret simply through visualization. As such, it is useful to explore some of the quantification measures social network analysis allows us to employ.

Incidentally, the presence of methods used to capture precisely these ideas is one of the benefits social network analysis provides beyond the results that conventional statistical methods can convey. Centrality and centralization are two of the oldest and most conceptually powerful concepts pertaining to social network analysis. The concept of centrality refers to the positions of individual vertices (i.e., actors) in the network and the concept of centralization characterizes the network as a whole. An actor is considered central to the extent that they are involved with other actors in the network and a network is consider highly centralized if there is a clear boundary between the center and the periphery (de Nooy et al., 2005).

\section{Degree Centrality}

According to Wasserman and Faust (1994), central actors are those actors that are extensively involved in relationships with other actors in the network. In viewing the centrality of an actor as being the extent to which information may easily reach them, it is clear that the number of sources accessible to a person, the more central their location. Thus, the simplest way to look at actor centrality is to assess what is called the degree 
centrality of the vertices which is simply measured as the degree value of the vertices as compared to the degree variation of all other vertices. Extending the notion of degree centrality further, we can look at the degree centralization of a network. The degree centralization of a network is the variation in the degrees of the vertices divided by the maximum degree variation which is possible in a network of the same size (de Nooy, 2005).

However, under these measures, we run into the same problem that the density of a network poses (i.e., the overall size of the network can interfere) in that the overall connectivity patterns of the network can cloud our interpretation. For example, a component with only four actors, one of whom has a high degree centrality while the others have a low degree centrality is difficult to compare to an actor in a much larger component with a lot of other actors and a great variation in degree values. Likewise, the degree centralization of individual vertices does not conceptually provide a precise indication of how the subgroups come together and therefore on weakly measures the centralization of the network as a whole.

\section{Closeness Centrality}

A better measure of an actor's centrality must include an assessment of their reachability. Earlier we looked at the reachability of a network through the extraction of components and bicomponents. However, we can take a closer look at the connectivity by assessing the distances between vertices. In general, the distance between any two 
vertices is based upon the number of lines (also known as steps) connecting two vertices. As there can be multiple paths present, the shortest path between two vertices is referred to as a geodesic.

Incorporating the concept of distance between nodes, we can assess another index of centrality called closeness centrality. The closeness centrality of a vertex focuses on how close an actor is to all the other actors in the set and is measured by the number of other vertices divided by the sum of all distances between the vertex and all others (de Nooy et al., 2005). Since it is not possible to assess the closeness centrality of a vertex when a network is not connected by path standards (i.e., contains multiple components) and the global network for all time blocks combined is the only one that entirely captures the overlaps for all times, we will explore the closeness centrality of the largest component for all time blocks combined.

To compute the closeness centrality indices for the largest component of the network for all time blocks combined, we used Pajek to create a vector. The centralization score for the entire component was calculated to be .2150. Table 4.9 provides the frequency distribution of centrality scores obtained through this process. Closenesss centrality scores are continuous rather than discrete, and therefore, in order to use visualization as an aid in the interpretation process, it is helpful to turn the vector into a partition to explore attribute patterns of the various centrality scores of the nodes. Figure 4.13 provides the image of the vector partitioned into 10 intervals.

As Figure 4.13 shows, the visual image of the partitioned centrality scores also provides us with the unique identification number for the actor that each node represents. Thus, we can use the identification codes to qualitatively assess patterns of attributes that 
relate to an actor's location within the main component. Three patterns that emerged relating to the centralization scores were a relationship between affiliation with Institution “Q” and a central location, affiliations with institutions by regional designations (i.e., only affiliated with institutions in the United States, only affiliated with institutions in Canada, only affiliated with institutions outside the United States and Canada, etc.), and predominant use of a certain type of methodology.

\begin{tabular}{|c|c|c|c|c|}
\hline Vector Values & Frequency & Freq\% & CumFreq & CumFreq\% \\
\hline $0.0000 \ldots 0.1022$ & 1 & 0.1079 & 1 & 0.1079 \\
\hline $0.1022 \ldots 0.1125$ & 1 & 0.1079 & 2 & 0.2157 \\
\hline 0.1125...0.1228 & 9 & 0.9709 & 11 & 1.1866 \\
\hline $0.1228 \ldots 0.1331$ & 20 & 2.1575 & 31 & 3.3441 \\
\hline $0.1331 \ldots 0.1433$ & 13 & 1.4024 & 44 & 4.7465 \\
\hline $0.1433 \ldots 0.1536$ & 72 & 7.7670 & 116 & 12.5135 \\
\hline 0.1536...0.1639 & 73 & 7.8749 & 189 & 20.3883 \\
\hline $0.1639 \ldots 0.1742$ & 64 & 6.9040 & 253 & 27.2923 \\
\hline $0.1742 \ldots 0.1844$ & 145 & 15.6419 & 398 & 42.9342 \\
\hline 0.1844...0.1947 & 116 & 12.5135 & 514 & 55.4477 \\
\hline $0.1947 \ldots 0.2050$ & 110 & 11.8662 & 624 & 67.3139 \\
\hline $0.2050 \ldots 0.2153$ & 104 & 11.2190 & 728 & 78.5329 \\
\hline $0.2153 \ldots 0.2255$ & 109 & 11.7584 & 837 & 90.2913 \\
\hline $0.2255 \ldots 0.2358$ & 33 & 3.5599 & 870 & 93.8511 \\
\hline 0.2358...0.2461 & 30 & 3.2362 & 900 & 97.0874 \\
\hline $0.2461 \ldots 0.2564$ & 10 & 1.0787 & 910 & 98.1661 \\
\hline $0.2564 \ldots 0.2666$ & 6 & 0.6472 & 916 & 98.8134 \\
\hline 0.2666...0.2769 & 7 & 0.7551 & 923 & 99.5685 \\
\hline 0.2769...0.2872 & 3 & 0.3236 & 926 & 99.8921 \\
\hline $0.2872 \ldots 0.2975$ & 1 & 0.1079 & 927 & 100.0000 \\
\hline Total & 927 & 100.000 & & \\
\hline
\end{tabular}

Table 4.9 Frequency Distribution of Centrality Scores 


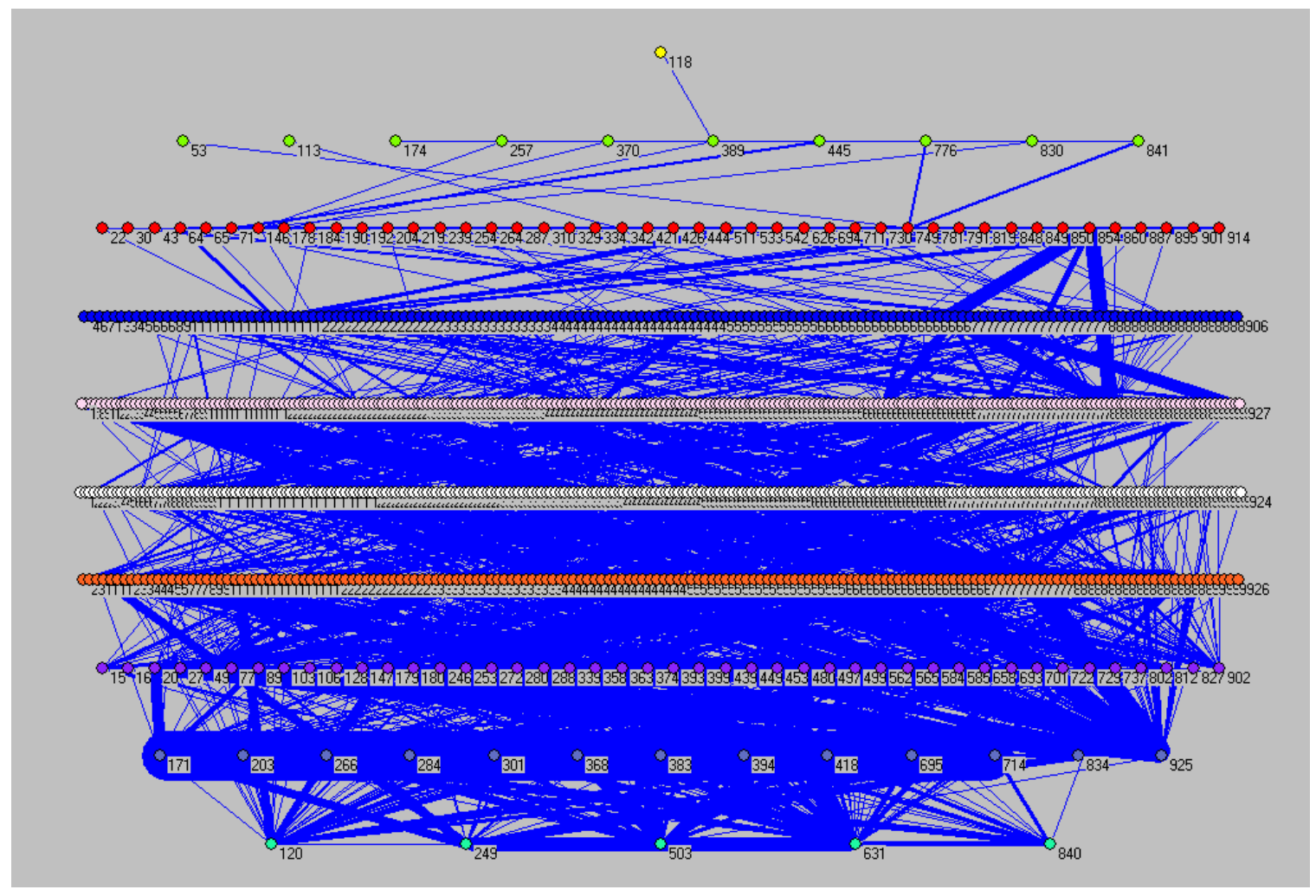

Figure 4.13 Visual Image of the Partitioned Centrality Scores of the Largest Component

To explore how these attributes are affiliated with positions in the network, null partitions were created in Pajek for each of the themes. In order to find the overlap between centrality scores and certain attributes, we ran a Cramer’s V, Rajski command for: 1) centrality scores and regional institutional affiliations, 2) centrality scores and affiliation with Institution “Q,” 3) centrality scores and methodological tendencies, 4) methodological tendencies and regional institutional affiliations, and 5) affiliation with Institution “Q” and methodological tendencies. While the attribute data was very sparse, some notable themes are definitely apparent. 
Although the attribute information clearly possesses a lot of missing data, it is important to highlight a few important points pertaining to the results of these overlap assessments. First, the analysis of those whose full institutional affiliation history is known suggests that those actors who are most central are primarily affiliated with institutions in the United States only. Four of the five "most” central actors have only been affiliated with United States institutions while the fifth has been affiliated with institutions in both the United States and Canada. In addition, 10 of next 13 "most" central actors have been affiliated with institutions in the United States only, while two more have been affiliated with the United States and other countries.

Secondly, all five of the "most central” actors possess institutional affiliations with Institution “Q." It should also be noted that four of these five actors currently serve as faculty members at Institution “Q." Moreover, 11 out of the next 13 “most” central actors also possess institutional affiliations with Institution "Q."

In regard to methodological tendencies, and all of the other measures (i.e., centrality partitions, affiliation with Institution “Q,” and regional institutional affiliations), several somewhat subtle patterns can be discerned. Of the 18 most central actors in the largest component, 14 identified themselves as primarily implementing quantitative methods, three identified themselves as using both qualitative and quantitative methods, and one actor's tendency was unknown. For the overlap between regional institutional affiliation and methodological tendencies, there appears to be a pattern in which those with institutional affiliations in the United States tend to employ more quantitative methods, while actors from Canada have a tendency to embrace more qualitative approaches. Finally, for those affiliated with Institution "Q," a majority were 
identified as primarily quantitative researchers, some expressed embracing both qualitative and quantitative methods, but only two identified themselves as primarily implementing qualitative methods.

\section{Betweenness Centrality}

Another approach to the concept of centralization is tied to the idea that a person is more central to the extent that he or she serves as an intermediary in terms of the connectivity of the network (de Nooy, 2005). This approach to centrality is referred to as betweenness centrality and attempts to capture the extent to which an actor is needed as a link in the chains of contacts that keep the network connected. Substantively, the betweenness centrality of a vertex is essentially the proportion of geodesics (shortest paths) between pairs of other vertices that include the vertex. Under these premises, the more an actor is needed as a "go-between," the more central his or her position is considered to be in the network.

Using Pajek, the betweenness centrality of the largest component of the coauthorship network for all times was calculated as .24. The distribution of betweenness centrality scores is provided in Table 4.10. In addition, like we did for the closeness centrality of the network, we also created a partition of the betweenness centrality scores for the network to aid in visualization. Figure 4.14 provides the image obtained through this process. 


\begin{tabular}{lrrrr}
\hline Vector Values & Frequency & Freq $\%$ & CumFreq & CumFreq $\%$ \\
\hline & & & & \\
0.0000 & 651 & 70.2265 & 651 & 70.2265 \\
$0.0000 \ldots 0.0272$ & 229 & 24.7033 & 880 & 94.9299 \\
$0.0272 \ldots 0.0544$ & 23 & 2.4811 & 903 & 97.4110 \\
$0.0544 \ldots 0.0816$ & 16 & 1.7260 & 919 & 99.1370 \\
$0.0816 \ldots 0.1088$ & 1 & 0.1079 & 920 & 99.2449 \\
$0.1088 \ldots 0.1361$ & 2 & 0.2157 & 922 & 99.4606 \\
$0.1361 \ldots 0.1633$ & 2 & 0.2157 & 924 & 99.6764 \\
$0.1633 \ldots 0.1905$ & 1 & 0.1079 & 925 & 99.7843 \\
$0.1905 \ldots 0.2177$ & 0 & 0.0000 & 925 & 99.7843 \\
$0.2177 \ldots 0.2449$ & 2 & 0.2157 & 927 & 100.0000 \\
Total & 927 & 100.000 & & \\
& & & & \\
\hline
\end{tabular}

Table 4.10 Betweenness Centrality Scores

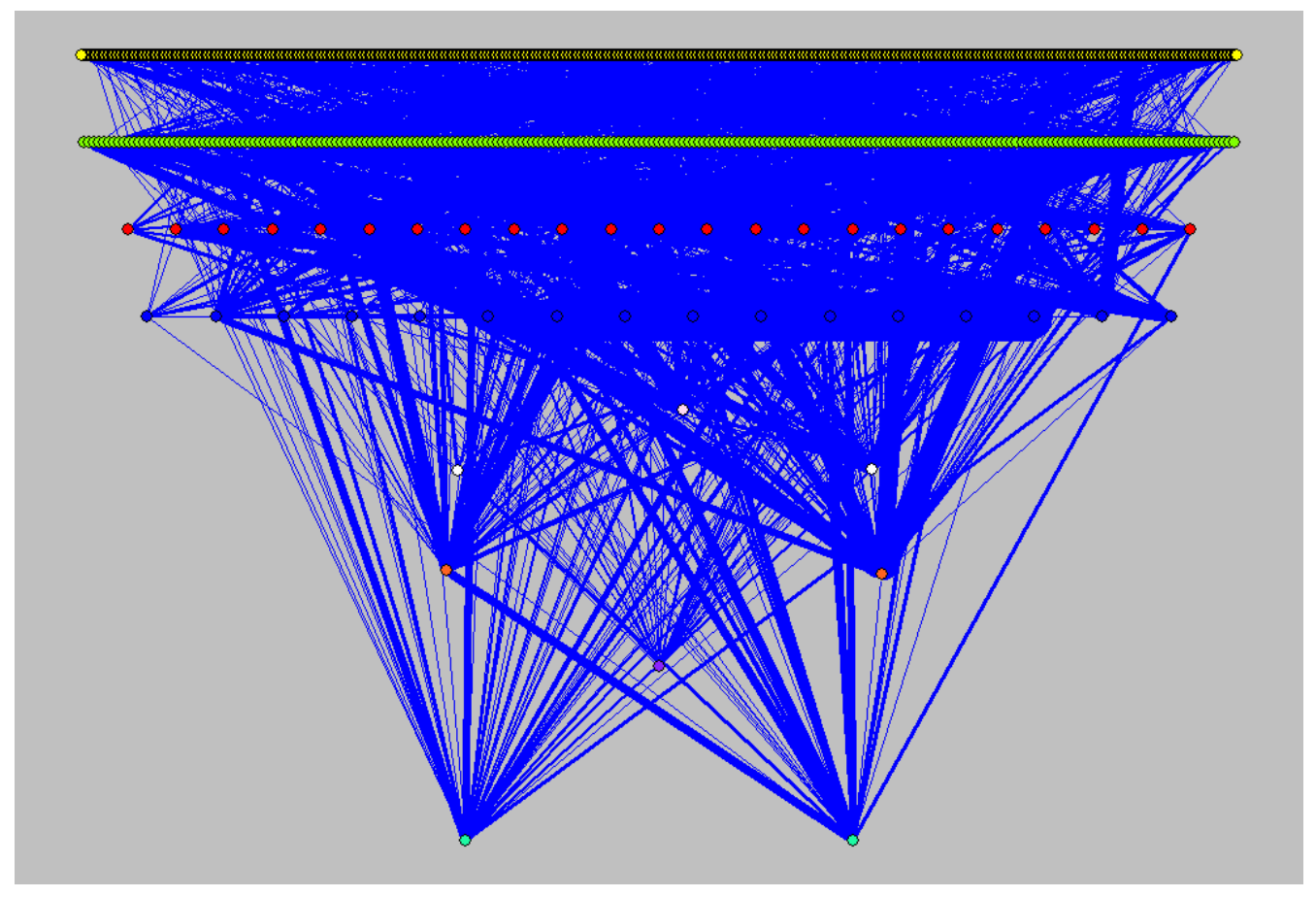

Figure 4.14 Visual Image of Betweenness Centrality for Largest Component 
In order to get a sense of who the actors with high centrality are and the attributes that might have led to such positions in the network, we used the unique identification numbers and the attribute spreadsheet to draw some connections between these properties. One of the attributes that all of the nodes in the four clusters with the highest betweenness centrality is that the actors have all been active in the field for a minimum of five years, with three of the eight actors having been in the field for 25 years or more. The actors all also appear to have a relatively high number of unique coauthorship ties (i.e., high degree values) falling in the highest $2 \%$ of the distribution for number of unique coauthors.

In addition, each of the actors appears to be an intermediary between clusters of relatively cohesive subgroups. For example, one actor who possesses institutional affiliations with institutions in both Canada and the United States appears to play an important role in connecting a number of actors with institutional affiliations in predominantly Canadian institutions and with institutional affiliations with schools in the United States. Another actor appears to be a connector between a number of specializations, has self-identified as specializing in both research design and statistics, and connects a lot of actors affiliated with Institutions "I" and "K."

In order to get a better sense of how both closeness centrality and betweenness centrality in the main component are related to the various themes we have identified, we exported the Pajek file for all vectors and partitions generated in this stage of the analysis to an SPSS file. Although the data was missing a lot of values for each of the variables and therefore must be interpreted very cautiously, we ran a correlation for all the themes and the two centrality measures. Table 4.11 provides the obtained correlations. 


\section{Correlations}

\begin{tabular}{|ll|r|r|r|r|}
\hline & & Method & $\begin{array}{c}\text { Institution Q } \\
\text { Affiliation }\end{array}$ & $\begin{array}{c}\text { Closeness } \\
\text { Centrality }\end{array}$ & $\begin{array}{c}\text { Betweenness } \\
\text { Centrality }\end{array}$ \\
\hline Method & Pearson Correlation & 1 & $.259^{\star \star}$ & $.264^{\star \star *}$ & $.468^{\star \star}$ \\
& Sig. (2-tailed) & & .000 & .000 & .000 \\
& $\mathrm{~N}$ & 927 & 927 & 927 & 927 \\
\hline Institution Q Affiliation & Pearson Correlation & $.259^{\star \star}$ & 1 & $.363^{\star \star *}$ & $.253^{\star \star}$ \\
& Sig. (2-tailed) & .000 & & .000 & .000 \\
& $\mathrm{~N}$ & 927 & 927 & 927 & 927 \\
\hline Closeness Centrality & Pearson Correlation & $.264^{\star \star}$ & $.363^{\star \star *}$ & 1 & $.370^{\star \star}$ \\
& Sig. (2-tailed) & .000 & .000 & & .000 \\
& $\mathrm{~N}$ & 927 & 927 & 927 & 927 \\
\hline Betweenness Centrality & Pearson Correlation & $.468^{\star \star}$ & $.253^{\star \star *}$ & $.370^{\star \star}$ & .000 \\
& Sig. (2-tailed) & .000 & .000 & 927 & 927 \\
\hline
\end{tabular}

${ }^{\star \star}$. Correlation is significant at the 0.01 level (2-tailed).

Table 4.11 Correlations for Emergent Themes and Centrality Measures

As was mentioned earlier, these correlations are not meant to infer conclusive results but rather identify important areas for further exploration. To aid in the interpretation of these correlations, we want to explicitly identify how many cases were included within each theme. Within the main component, 56 actors of the total 927 were identified as possessing an institutional affiliation with Institution “Q.” Of the known regional institutional affiliations (430 out of 927), 279 were identified as actors with only United States’ institutional affiliations, 56 were identified as actors with only Canadian institutional affiliations, 11 possessed institutional affiliations in both the United States and Canada, 72 were identified as having institutional affiliations only outside the United States and Canada, and 12 were identified as having a combination of different sorts. Finally, because information on methodological preferences was obtained from only 
those actors who had participated in the sampling procedures, only 71 actors were included in the analysis on methodological preferences (the 5 other actors were not located within the main component).

\section{Bridges}

One common contention by network analysts is that the number of social of ties a person has is important in terms of access to receive and opportunities to spread information. However, in addition to the number of ties a person possesses, it is important to consider how the cohesive subgroups figure into a person's structural location. As Burt (1992) and others have argued, an actor who has lots of ties within a group is likely exposed to the same information over and over again, whereas ties outside the actor's group potentially offers access to more diverse information. Therefore, network analysts suggest that it is not only necessary to pay attention to the people an actor is connected to, but also to pay attention to the ties between the actor's contacts. That is, as de Nooy et al. (2005) articulated, “A person who is connected to people who are themselves not directly connected has opportunities to mediate between them and profit from his or her mediation” (p. 138).

From these arguments, it is useful to look at which actors are in the best position to have access to diverse information thus profiting from his or her social ties. Moveover, the presence of multiple actors who do possess such advantageous structural positions, implies that the network contains bottlenecks where the flow of information is vulnerable and dependent upon these focal actors. Thus, to identify actors who hold structurally 
advantageous positions, we must locate which ties and which vertices are indispensable in order for the network to remain connected. Social network analysis allows us to do precisely that through the concepts of bridges.

A bridge is essentially any line whose removal increases the number of components in the network. The removal of any line may change the connectedness of a network as can the deletion of a vertex. In fact, the removal of a particular vertex can often have an even more detrimental effect particularly if the vertex has a high degree value. Hence, a cutpoint or cut-vertex is a vertex whose deletion increases the number of components in the network. In Pajek, the Bi-components command can be used to find bicomponents, bridges and cutpoints in the network. Table 4.12 provides the frequency distribution for the number of bridges to which each vertex belongs. To clarify, about $85.98 \%$ of all of the vertices in the main component belong to one bridge while one vertex belongs to 20 bridges. 


\begin{tabular}{crrcc}
\hline \# of Bridges & Freq & Freq\% & CumFreq & CumFreq\% \\
\hline 1 & 797 & 85.9763 & 797 & 85.9763 \\
2 & 80 & 8.6300 & 877 & 94.6063 \\
3 & 16 & 1.7260 & 893 & 96.3323 \\
4 & 8 & 0.8630 & 901 & 97.1953 \\
5 & 5 & 0.5394 & 906 & 97.7346 \\
6 & 4 & 0.4315 & 910 & 98.1661 \\
7 & 1 & 0.1079 & 911 & 98.2740 \\
8 & 4 & 0.4315 & 915 & 98.7055 \\
9 & 5 & 0.5394 & 920 & 99.2449 \\
11 & 1 & 0.1079 & 921 & 99.3528 \\
12 & 1 & 0.1079 & 922 & 99.4606 \\
13 & 1 & 0.1079 & 923 & 99.5685 \\
14 & 1 & 0.1079 & 924 & 99.6764 \\
15 & 1 & 0.1079 & 925 & 99.7843 \\
18 & 1 & 0.1079 & 926 & 99.8921 \\
20 & 1 & 0.1079 & 927 & 100.0000 \\
& & & & \\
Sum & 927 & 100.0000 & & \\
\hline
\end{tabular}

Table 4.12 Frequency Distribution of Bridges per Vertex

To draw some connections between the attributes of the actors and the number of bridges to which they belong, we used the unique identification numbers of those actors belonging to more than 11 bridges to look for patterns related to the actors' attributes. One common attribute for all of these actors is that they have been productive in the field for a number of years. A second common attribute of all of the actors is that many of them serve as important vertices in terms of connecting one relatively cohesive subgroup to another.

For example, the actor who belongs to 20 bridges, serves as an intermediary between a relatively cohesive subgroup of actors with institutional affiliations in the United States only (many of who possess a specific institutional affiliation with 
Institution "Q") and a somewhat connected subgroup of actors with institutional affiliations in Canada only. Incidentally, the actor possesses institutional affiliations with schools in both the United States and Canada.

The actor who belongs to 18 bridges manifests similar traits in that he/she serves as an intermediary of a relatively cohesive subgroup of actors with institutional affiliations located primarily in the United States and a couple of actors who are primarily affiliated with institutions in Canada. The difference between this actor and the one described above is the fact that this particular actor only possesses affiliations with institutions in the United States, while his/her ties to the Canadian constituents are mediated by a couple of actors who possess institutional affiliations in both the United States and Canada. Moreover, the actor belonging to 18 bridges also possessed a number of ties to actors whose specialization was physical education only. Thus, the actors that fall into this category do not possess many ties to the rest of the actors in the network outside of the subgroup making the actor with 18 bridges a broker between the main network and their subgroup of physical educators.

The actors belonging to 15,13 , and 12 bridges all possess a similar trait in that in addition to publishing in the field of sport management, they also publish on general business topics (i.e., management and marketing) entirely unrelated to sport. As such, while they play important roles in the sport management world, they are tied to a number of actors who do not regularly publish in sport management. Thus, many of the people to which they are tied, do not possess much overlap between the attributes of the other 
actors in the field. As such, the actors belonging to 15, 13, and 12 are not only serving as brokers of actors in varying specializations, they are actually serving as brokers to actors in entirely different fields of study.

The actor who belongs to 14 bridges is unique in the fact that he/she possesses institutional affiliations with schools both within the United States and outside the United States and Canada. Moreover, he/she specializes in a number of different and actually possesses a doctoral degree in a field that is quite different from most of the actors' degrees in the field. Many of this actor's bridges are actually ties to individuals in another field and/or ties to actors located at institutions outside the United States and Canada. Thus, while some of the actor's contacts have published with one another, most have not published with many (if any) other actors connected to the main component.

\section{Conclusion}

As was articulated in the beginning of this chapter, the design of this chapter was intended to provide the reader with a transparent overview and intuitive representation of how the themes were assessed and developed. In all reality, the assessments presented barely even touch upon the calculations and evaluations that could be carried out with the dataset. Moreover, due to the time and resource constraints some of the data that is included in the analysis is extremely limited and therefore can only be loosely interpreted for predictive qualities. Consequently, the results described are not meant to serve as conclusive evidence to the themes we developed but rather serve as an exploration into viable and beneficial topics for future studies. 


\section{CHAPTER 5}

\section{DISCUSSION}

Several debates that have continually surfaced in the field of sport management were raised in Chapter 1, and the values of working through these "growing pains" that often arise as new fields emerge were highlighted. Among the points suggested, we noted that in pursuing, as Amis and Silk (2005) put it, “an academic landscape that is not dominated by any single overarching metanarrative that marginalizes and obfuscates alternative approaches,” researchers in the field should be concerned about the political, social, and ideological patterns that may exist in terms of individuals’ scholarly interactions with one another (p. 358). Engaging such a perspective, begs the exploration of several important questions. For example, how is the advancement of knowledge in the field being collectively pursued? Moreover, are there specific features of the social structure of the community of sport management scholars that impede the ability of innovative ideas from being integrated into the idea space and practices of the discipline? Informal conversations and philosophical debates surrounding these questions frequently occur, and indeed, a number of scholars have encouraged and even pursued empirical investigations into these matters (e.g., Costa, 2005). Nevertheless, this study adopted a different approach. Rather than asking ourselves specifically what the current status of the idea space and practices are comprised of, we sought to understand from a 
socio-structural standpoint how the current voids and dominant forms arose in the first place. As such, one focus of the study took a historical look at how, when, and from whom the intellectual foundations of the field emerged through the lens of exploratory social network analysis. Hence, the first part of the results section in Chapter 4 and the early parts of the discussion in this chapter are essentially a story of the evolution of the publication network.

Through this study we also sought to investigate whether or not potential sociostructural barriers exist that may inhibit the spread of innovative ideas and practices across the network of sport management researchers. Although the data analyses in their current state are not conducive for either a conclusive or a predictive model of diffusion, we address the specific themes about the network raised in Chapter 4 under the microscope of several theoretical ideas related to diffusion models. Consequently, the ideas suggested in the latter half of this chapter are not intended to be a story of diffusion, but rather a story discussing the structure upon which diffusion must have occurred.

In the final sections of this chapter, we discuss some additional theoretical, methodological, and practical implications of the study. We also provide an overview of the limitations and delimitations incurred. Finally, as this study was designed to lay the foundation for future studies, we conclude with some suggestions for future research directions.

\section{General Collaboration Trends and Evolution of the Field}

The findings that coauthorship in general and the number of coauthors per publication have increased over the years is consistent with findings in studies pertaining 
to other academic fields (e.g., Moody, 2004; Persson, Glanzel, \& Danell 2004); although perhaps comparatively low in regard to the physical sciences. The fact that several individuals who are newer to the field and have nearly reached the number of publications of many of the scholars who have been in the field a long time, might throw off the argument that productivity and time in the field are highly correlated. However, some have argued that the growth in numbers of papers per author can also be an effect of collaboration rather than individuals becoming more productive (Persson et al., 2004). Moreover, the argument could also be made that over the years the field has evolved such that some institutions are now providing more resources and time to aid in greater productivity. Likewise, perhaps in some cases, institutions are even demanding a productivity level that far exceeds the levels of expectations experienced by the early scholars in the field.

Furthermore, the fact that a great majority of the work being published in the field is being produced by a very small minority of people is also consistent with the results of studies conducted in other fields. For example, Moody (2004) found that of all authors that appear in the Sociological Abstracts database, 66\% appear only once and an additional $15 \%$ only appear twice. As such, these numbers are important to keep in mind when asking how structural and institutional factors play into the work being disseminated, interpreted, cited, and extended in the field's idea space. In other words, locating where the actors with the high productivity rates are positioned in the overall structure can be of extreme value in terms of understanding the social structure of the field. 
As noted in Chapter 4, many of the isolates and structurally disconnected actors in the network are actually actors who published only once or twice in the field. In many cases, these isolated actors are either practitioners who published as a single author or groups of scholars who mainly publish in other fields such as business, management, marketing or economics and happened to publish a study related to sport management in one of the sport management journals (e.g. Journal of Sport Management or Sport Marketing Quarterly). As a side note, we should mention that while it has been suggested that sport management often borrows ideas from other fields of study (e.g., Slack, 1998), the results of this study indicate that we should also recognize that, in many ways, the field actually "borrows" scholars from other fields. That is to say, it is not just the ideas of scholars in other fields that are contributing to our idea space, but the actual participation of scholars in other fields contributing to the knowledge being generated in the field.

Nevertheless, because such scholars do not often teach in sport management programs and/or frequently participate in other opportunities for face-to-face interaction, they are not likely to have had a huge impact in terms of influence aside from being cited or having their frameworks used by those who are more structurally central in the field. Likewise, those actors who are structurally central but have only published once or twice in the field may also be construed as having very little influence on the field. That is not to suggest that they will not greatly influence the field in the years to come, however, their central positions tend only to be a result of being tied to central actors in the network. Thus, in many cases, their central positions were obtained via collaboration with an advisor who is central. 
So, who are the actors in the field who have likely had great influence? Moreover, historically, how have they come together to constitute the academic idea space we call sport management? As shown by the evolutionary visualizations provided in Chapter 4, over the past 20 or so years, the field of sport management has grown immensely in a number of regards. In addition to the number of actors participating in the field, there is also a general trend in the overlaps of actors who collaborate with one another. That is, in looking at the visual depictions of the structures over the years, an obvious trend is the growth of one dominant component of actors present in the global network structure.

These features raise the questions: What circumstances and interactions helped 1) stimulate the exponential growth of the field and 2) build the bridges between the early separated clusters to help form a more cohesive collaboration structure? According to Crane (1988), periods of rapid growth can be attributed to a couple of factors related to the cognitive growth and social interaction. Early on, the emergence of a field is dependent upon several researchers making a shift in their research priorities and whose work in the emerging discipline attracts new scientists to come on board. Later, growth in the field is often related to several lead scientists who recruit or draw new members and students to the field.

Indeed, these patterns appeared to hold true in the case of our obtained network structure. In the early time blocks, a number of very distinct clusters of actors went about contributing to the idea space in isolation of one another. The nature of these groups tell a story of a number of scholars from a variety of disciplines attempting to break into a new domain of study. Interestingly, from even the earliest periods we can see cause for the longstanding debates pertaining to the establishment of the appropriate "house" (i.e., 
physical education or business) for sport management. A number of early actors in the field possessed backgrounds in physical education while many others migrated to sport management from business-related focuses (e.g., management, marketing, and economics). In addition, the clusters of individuals also suggest other thematic considerations for the early broken coauthorship structure such as clustering based upon regional and institutional affiliation variables.

Over the years, a number of institutional forces such as the establishment of NASSM, the emergence of several new journals, and presentations at academic conferences helped create both a sense of identity as well as more formal and informal opportunities for scholarly collaboration. Moreover, a number of sport management programs began to appear all over North America. While it is likely all of these factors contributed to the steady growth in collaboration overlaps, the introduction of new programs and hence the recruitment of new faculty members, may actually have had the most significant influence on the unique coauthorship patterns and bridging of the various clusters.

In fact, the observed network structure suggests that it is likely some of the greatest structural shifts can be attributed to three main factors. The first factor is actually the fracturing of one group of scholars with primarily Canadian institutional affiliations into two distinct clusters each characterized by the presence of future structural stars. Conforming to Crane's (1988) model in which it was suggested that in the secondary stages of a field's development, "a few highly productive scientists set priorities for research, recruit and train students who become their collaborators, and maintain informal contact with other members of the area” (p. 40). In terms of the fracture between 
these two Canadian "stars," one important substantive feature stands out. One of the stars seemingly adopted and trained his/her students under a methodological paradigm that embraced more postpositivistic, qualitative philosophies and procedures while the other star appeared to embrace a tendency toward positivistic, quantitative methods.

The second dramatic structural shift appears to actually have begun when the structural star from Canada who embraced quantitative methods moved from Canada to the United States and became affiliated with Institution "Q." Around the same time block, another structural star from the United States with a background in physical education also became affiliated with Institution "Q." While the two stars at Institution "Q" did not coauthor together, it appears that a number of the students at Institution "Q" mutually collaborated with both of the stars. In fact, around this time, Institution "Q" became extremely industrious in terms of educating a number of students who later went on to become very productive in the field.

The third factor is similar to what Freeman (2004) found in an analysis of the social construction of social network analysis. It seems that much of the bridging in the network occurred as a result of faculty and student migration from one institution to another. In particular, the migration of students from Institution “Q” to other institutions appeared to play a large role in both the bridging of many clusters of actors across the network and as well as the presence of a relatively cohesive core of actors within the largest component. Interestingly, for whatever reason, the students from Institution "Q" appeared to have maintained many of their cohort ties from their doctoral days while also building new coauthorship ties with other scholars at their subsequent institutions. 
In spite of the growing cohesiveness and volume of the main component in the coauthorship network, a number of seemingly large structural gaps also appear in the network. Through our qualitative exploration of the actors' attributes, we made a number of interesting discoveries. For one, although a number of weak formal ties exist, and likely, a number of informal relationships are present, in terms of formal coauthorship patterns, there is a very distinct structural divide between individuals with institutional affiliations located primarily in Canada and actors with institutional affiliations located primarily in the United States. Due in part to the volume of actors from the United States in comparison to the number of actors in Canada, the measures used to assess centrality in Chapter 4 indicated that, in many ways, those with institutional affiliations primarily in Canada are actually relegated to the periphery of the main component if they are connected at all.

Intriguingly, the structure of the network characterized by those with primarily Canadian affiliations is not particularly dense. In comparison to some of the other clusters within the main component, it might actually be construed as being a rather sparse and fractured cluster. In addition, of the actors in the Canadian cluster whose methodological preferences are known, a number of them identified themselves as primarily carrying out qualitative studies. In fact, although there were significantly fewer actors known to have primarily Canadian institutional affiliations, preferences for qualitative research appears to be proportionally higher for these actors than actors who were identified as only possessing institutional affiliations in the United States. It could be argued that part of the 
reason for the lower connectivity levels in this cluster is simply related to the nature of qualitative research. As Moody (2004) suggested, perhaps authors who embrace more qualitative approaches are less likely to coauthor in general.

Regardless of the less cohesive nature of the cluster, an interesting connection can be drawn between the fact that many of the self-identified qualitative researchers are located in the periphery of the network and the notion that certain socio-structural barriers may be present that impede new approaches from being integrated into the idea space. As was mentioned in Chapter 1, Amis and Silk (2005) alluded to the fact that sport management has been slow to acknowledge and adopt new and different methodological approaches in comparison to other fields. Consequently, although the themes developed through this study are not conclusive, they do provide veritable evidence that certain socio-structural barriers to the diffusion of ideas may be present in the field of sport management. To delve into these ideas further, it is useful to engage in a theoretical discussion about what the obtained network structure may hold for the diffusion of innovations throughout the network.

\section{Socio-Structural Barriers for Diffusion of Innovative Ideas?}

Epidemiological models for disease diffusion and models of social contagion have recently started incorporating social network ideas into how patterns of network ties affect diffusion through a population of actors. While having a structural map of the social networks of a population of actors may not be able to precisely predict which individuals will innovate or adopt an idea first, they can help assess how diffusion may 
play out based upon the network position of the innovator or first adopter. In these regards, according to White, Owen-Smith, Moody and Powell (2004):

Structural cohesion should matter to individuals and groups because it scales the linkage of specific actors to one another, facilitates the flow of information and exchange through multiple channels, allows the cross-checking of information as a basis for establishing reliability and trust, and supports the robustness of social groups and their adaptive resilience through multiconnectivity” (p. 99).

In Chapter 1, we discussed some social network features that are theoretically tied to diffusion rates including network distance, clustering, multiple paths, and timing. One underlying requirement of all of these features is related to the general connectivity of a network in that in order for influence or a message to be carried out via the structural networks that are present, the nodes must have a path connecting them. We assessed the connectivity of the global network in a number of ways, one of which was a simple assessment of the components present. As Scott (2000) noted,

The members of a component can, in principle, communicate with one another, either directly or through chains of intermediaries. Isolates, on the other hand, have no such opportunities. The pattern of components found in a graph-their number and size — can, therefore, be taken as an indication of the opportunities and obstacles to communication of the transfer of resources in the associated network (p. 102).

The assessment of components indicated that 124 isolates were present in the network, and beyond that, 143 components were present. The largest of the components 
consisted of 927 vertices which accounted for about $62 \%$ of the authors included in the network. Focusing in on the one large component, we then assessed the network properties in greater detail.

In terms of network distance, it is suggested that the shorter the distance between two nodes, the greater the likelihood the innovation will migrate from the first node to the second node. One measure that is particularly useful in terms of understanding the connection between network distance and diffusion is closeness centrality which fundamentally assesses how close an actor is to all other actors in the network. According to de Nooy et al. (2005), in general, if the position of the first adopters is centrally located in the network, the likelihood for diffusion throughout the network is much greater.

When we calculated the closeness centrality for all vertices in the main component, several themes emerged. One of the most noticeable themes was the fact that 10 of the 13 most central actors in the system all possessed institutional affiliations with Institution “Q," and four of the five most central actors are actually current faculty members at Institution “Q.” A second strong theme that emerged was the possible presence of a social-structural gap between actors in the United States and Canada. This theme was evidenced by the fact that only one actor of those known within the 62 "most central” positions possessed solely Canadian institutional affiliations, while 34 possessed solely affiliations with institutions in the United States and 4 possessed affiliations with both the United States and Canada. Moreover, 14 of the 18 most central actors were affiliated with only institutions in the United States.

Interestingly, these themes appeared to carry out in terms of the clustering patterns in the network as well. When examining the clustering patterns of the network, it 
appeared that there has been very little between group coauthoring as scholars from the United States tend to coauthor with each other while scholars from Canada have a tendency to publish together. In addition, the patterns of cohesive subgroups (and as such the presence of multiple paths) also appear to align with these two themes.

Furthermore, while there were several relational bridges stretching between the two subgroups, the way in which the notion of timing plays into the structure of the network also provides robustness to the two identified themes. For example, the few ties that stretch between the subgroups of actors in the United States and actors in Canada, were relatively weak in intensity and occurred early on in the development of the network. Hence, the likelihood of diffusion through these ties today is probably not all that strong. Moreover, the strength of ties within the subgroups (particularly between those actors possessing affiliations with Institution "Q”) was much stronger than the strength of the ties between the subgroups.

Since the data for actor attributes was very limited as it contained a lot of missing data, the results of the analyses conducted in this study should be interpreted very cautiously. Nevertheless, the pervasiveness of these two themes throughout the results of this study provide a strong argument that affiliation with Institution "Q," the clustering of regional institutional affiliation, and the centrality of the actors in the network should be looked into further in future studies. In the event that these themes do hold true when the analyses can be deemed more conclusive, both may underscore the presence of some very strong socio-structural barriers to the diffusion of innovations throughout the network of researchers in the field of sport management. 
Numerous studies related to the idea of "Collective Memory" (i.e., why some things are retained while others are forgotten) have shown that external factors as well as the qualities of works themselves affect what will be retained in a culture (Swidler \& Arditi, 1994). As Swidler and Arditi (1994) articulated, “It seems reasonable to believe that the authoritativeness of knowledge is grounded in patterns of social authority” (p. 311). Such ideas are consistent with other models of scientific knowledge generation; particularly Kuhn’s The Structure of Scientific Revolutions, Crane’s work on “invisible colleges," and many of the philosophical points introduced by the works of Foucault. Along the same lines as the argument that "what enters the literary canon depends on the interests of those who control it" (Swider \& Arditi, 1994, p. 309), it could be argued that possessing a central place in the network is one of the keys to getting your work published, recognized, cited, and extended.

If affiliation with Institution "Q" truly does have such a central, cohesive presence in the network, it may be that in order for an innovation to diffuse rapidly (or perhaps even at all), actors with an affiliation with Institution "Q" have to adopt (or at least "buy into" the ideas). Moreover, if there really is a structural gap between scholars in the United States and scholars in Canada whereby the scholars from Canada are relegated to the periphery, it may be difficult for ideas or practices that develop in Canada to diffuse through the network. Additionally, due to the fact that the connectivity in the more central parts of the network is somewhat stronger than the connectivity in the periphery (particularly between the Canadian scholars), it could be argued that while there tends to be a lot of trading of ideas in the central core of the network, very little trading of ideas takes place in some of the peripheral clusters. 
In the case of qualitative research and other alternative methods, is it possible that because many of the scholars that have embraced these ideas for a long time were located in the periphery of the collaboration social structure, the adoption rate has been rather slow? While a conclusive exploration into the diffusion of postpositivistic methods in the field of sport management is outside the scope of this study, the results of this study suggest that the presence of socio-structural barriers quite possibly may have played a role in the adoption rate of qualitative methods in the field. With the presence of sociostructural barriers comes the potential for the exclusion of innovative ideas whereby "outgroup” members may have a difficult time getting their ideas accepted, remembered and utilized. Of particular note is the seemingly dominant presence of Institution "Q." As the students that graduate from Institution Q have likely all been exposed to, trained in, and likely gone on to practice and focus on the same sorts of ideas and methods, we should be especially aware of potential for groupthink to occur.

\section{Theoretical Implications}

Due to the exploratory nature of this study, the results reported do not necessarily build upon or extend sociological theories in the grander sense. However, when the results of the study are related back into theory about social networks and diffusion, they do provide a theoretical foundation for future testable hypotheses related to the idea space and patterns of diffusion in the field of sport management. In fact, with some additional archival data collection, and further analyses, the propositions suggested in this study can easily be tested for more conclusive results. 
Broadly speaking, this study incorporated two major thrusts in terms of extending the idea space in the field of sport management. The first thrust was essentially to critically reflect on the social construction of knowledge in the field of sport management. While previous studies and essays have critically analyzed and discussed the status and culture of the field, to date no study has examined the social structural development of the field from a social constructivist and a social network perspective. Incidentally, the secondary thrust of this study was to fundamentally introduce social network analysis as an alternative theoretical and methodological paradigm to the field of sport management.

\section{Methodological Implications}

Social network analysis is grounded on the premise that the patterning of social ties in which actors are embedded can have important consequences for those actors as well as for the social population as a whole (Freeman, 2004; Wellman, 1988). As such, network analytic studies often seek to uncover patterns of interaction, determine the conditions under which those patterns arose, and make attempts to identify their consequences. In terms of studying the generation of knowledge in a scientific field of inquiry, social network analysis provides an intriguing perspective about how a group of relatively disconnected scholars interacting with one another can over time come together to constitute the idea space of a specific field of study.

Although a lot of the basic theory and methodological techniques have been around for decades, social network analysis has been less discussed in mainstream literature for several reasons. The first is that the methods can be somewhat unwieldy and 
complicated to carry out and the technological advances and computer software have only recently provided more opportunities for in-depth analyses. Moreover, the software still requires a somewhat comprehensive knowledge of social network methodologies and specific training in certain elements of computer programming. In addition, general reluctance to embrace a network paradigm also exists because social network analysis entails a distinct approach to research. As Nohria (1992) articulated, to effectively employ a network perspective "means adopting a different intellectual lens and discipline, gathering different kinds of data, learning new analytical and methodological techniques, and seeking explanations that are quite different from conventional ones” (p.8).

Incidentally, social network analysis and sport management have two things in common: their interdisciplinary nature and their ability to bring together scholars from a wide range of disciplines. While social network analysis brings together sociologists, mathematicians, physicists, epidemiologists, and computer scientists, sport management serves as a mixing ground between such fields as communications, marketing, sociology, organizational behavior, psychology, human resource management and physical education. As sport provides a unique domain in which quantified performance records are easy to come by, and the interdisciplinary natures of both sport management and social network analysis cut across the boundaries of traditional disciplines, the linking of these two worlds could very well establish a veritable nexus for the collaboration scholars from a variety of fields.

As such, it was the desire of the researchers that the procedures carried out in this study serve to insightfully delineate the power and usefulness of social network analysis 
as a methodological paradigm. The methods employed and the analytical procedures carried out were described in great detail with the intent of providing a transparent and comprehendible depiction of some of the capabilities and measures that social network analysis has to offer. By tying these ideas to both a worthwhile and relevant topic for scholars in the field of sport management, we hope to attract both attention and legitimacy in the eyes of researchers for social network analysis to be employed in their future research endeavors.

\section{$\underline{\text { Practical Implications }}$}

In essence, knowing the social network structure of a system of actors can help us make informed decisions about where in the network innovations should be introduced to best facilitate the diffusion process (Moody \& Leahey, 2006). In terms of improving the overall structure of the coathorship network, it is not logical to expect individuals to break apart from their tendencies to collaborate with their former classmates, institutional colleagues, and collaborators with similar interests. Nevertheless, making people aware of the potential socio-structural barriers does provide a motive for them to make more conscious collaborative efforts toward bridging the structural holes. As noted by Moody and Leahey (2006), models of social networks and diffusion show that a relatively small number of connections between otherwise disconnected clusters of actors, can rapidly decrease the average distance between nodes and increase the number of unique paths. Thus, even if only a few central actors in the network find ways to bridge the socialstructural gaps, perhaps the rate and likelihood for the diffusion of innovations can significantly improve. 


\section{Limitations and Delimitations}

While by conventional standards, a response rate of 31-33\% might be considered low and not all that representative of the population at large, the representativeness of a sample for a study in social network analysis can be judged by a different sort of standards. In many traditional studies, the goal is to elicit a random sample of individuals under the premise that if every member of the population has an equal and independent chance of being selected, any differences that exist between the population and the sample should be small and unsystematic which can be attributed to chance rather than bias on the part of the researcher. However, since social network analysis seeks to understand the relationships between actors in the population random sampling is often not of interest or particular use for the researcher. Ideally, a relative census of the population is the best way to collect data for social network analysis. Nonetheless, in many cases, it is not possible to take measurements on all the actors in the relevant actor set and thus some sort of sampling techniques must be implemented.

Thus, as social network analysis focuses on the idea that structural patterns of relationships between actors in a system, an imperfect representation of the true underlying structure is based upon the extent to which actors who may indeed be relevant to the construct of interest are ignored. In other words, a dataset that is missing actors who in actuality serve as key people in the network can potentially portray a very distorted representation of the true structural configuration. Therefore, the structural "quality” of the participants in the study tends to be of greater importance than the quantity of participants. 
In the case of the sampling techniques used for this study, the "quality" or relative importance of the participants can in one regard be judged upon the extent of their research productivity in the field. That is, an actor who participates that has produced a number of publications in the field is considerably more important to the validity of the network structure obtained than say someone who has only published one time. Secondly, the "quality" or relative importance of a participant can be judged in terms of how connected they are to other actors in the network. In other words, an author who possesses a number of ties is likely to be structurally more important to the network of actors than one who can be construed as a social isolate. Finally, although there are other structural variables that factor into the "quality" of a participant (e.g., number of bridges they belong to, brokerage roles, etc), in terms of grasping an understanding of the evolution of the structure of the field, the relative importance of an actor can also be linked to the number of years they have been producing publications.

An assessment of the tables and figures in Appendix A gives us a general idea of the quality of the sample of the participants in these regards. In all reality, the robustness of the network is actually supplemented by the fact that although an actor in the system was not an actual participant, their ties to others in the system may have surfaced by virtue of their coauthors serving as participants. In addition, to supplement the data collected through the sampling process, the publications in five of the journals in which scholars in the most frequently publish were added to the dataset. The list of publications and their coauthors obtained through the sample of curriculum vitae was comprised of 
1,343 articles. The addition of the supplemental publications from the supplemental journals yielded an additional 737 publications for a grand total of 2,080 publications used in the analysis.

Consequently, an evaluation of how the actors who responded compare to the overall results provides a better illustration of the "quality" of the participants. For example, it is clear that the participants in the study tend to fall into the extreme end of the spectrum in that well over half of the respondents fall into the top $5 \%$ in terms of the number of articles they have published. Likewise, the distribution of unique coauthors for the respondents clearly falls near the higher end of the distribution for the entire network. Given that the distribution for all of the actors strongly suggests that a majority of the work in the field is being produced by a very small number of people, by overlapping a great deal with the top $5-10 \%$ of the total network distributions, it is likely that our sampling methods were able to capture many of the most "important” actors in these regards.

Since the actors' number of years in the field was calculated by the number of years that have passed since an author's first publication appeared in the network, the interpretation of this attribute can be clouded by the fact that an author could have published an article in the field and then never published again. Therefore it is more relevant to discuss the representativeness of the participants in terms of the respondents' composite average number of years in the field. The average number of years in the field for respondents was calculated to be 12.39 years, with the minimum being 1 year and the maximum being 33 years. 
Furthermore, when the network was assessed to identify the actors who had published more than 10 times in the field, 44 out of the 72 actors participated in the data collection. The fact that 28 actors were identified as falling into this category speaks to the robustness of the dataset obtained through the multiple sampling techniques. These 28 actors were identified as publishing more than 10 times in the field merely through the fact that their coauthors had participated or through the supplemental journal assessment.

In regard to the non-respondents and actors who responded but chose not to participate, several limitations should be highlighted. One of the greatest limitations in terms of using an email-based approach toward eliciting participants is the fact that there is no way of knowing whether the email sent went straight to a person's bulk or junk mail folders. Therefore, it is impossible to know which and how many of the non-respondents actually received the email. In addition, the turn around time for the email participation requests and the deadlines for analysis fell around many institutions’ spring breaks. Thus, it is entirely possible that a number of individuals did not respond based upon the timing of the data collection. Finally, even though the study was designed to ensure the anonymity of actors in the field, some scholars may have been leery to share such personal information about their activities in the field; particularly if they felt that their productivity in the field was minimal in comparison to other sport management researchers. In fact, this very scenario may have actually served as a filtering process for attracting more involved participants and weeding out those who have been less productive in the field.

A second concern of network analytic studies is the relationships that are chosen to represent the constructs of interest. For this study, the ties used to define the 
collaboration between actors in the system were based solely on formal coauthorship relationships. As such, some important examples of formal collaboration were neglected including: conference presentations, edited book chapters, and books.

While the curriculum vitae collected provided much of this information, due to time constraints and the accessibility of supplemental sources beyond the vitae necessitated their exclusion from the dataset at this time. Incidentally, the fact that many conference presentations tend to be either less formalized and finalized collaborative ideas, many of which are ultimately dropped or pursued in the form of a refereed publication may help offset some of the error introduced by their exclusion. In addition, it is likely that authorship on books tends to entail a lower rate of coauthorship in general and certainly the number of coauthors per publication. Moreover, the fact that sport management is still a relatively young field by many standards combined with the institutionalized pressure to publish research articles to meet tenure requirements, likely eliminates the opportunities for many of the researchers who are newer to the activity field. Thus, authorship on books is likely to be limited to those actors who have already established themselves as producers in the field and earned tenure; many of whose most likely collaborators are already tied to them via research publications.

Perhaps the most troubling of the excluded materials is that of book chapters in that it is an easier domain for up-and-coming researchers, students of advisors, and new actors to be introduced into the idea space of the field. Furthermore, it is possible that such book chapters may entail the collaboration between structural "stars" thus potentially bridging a structural hole in the obtained network. However, it could be 
argued that most book chapters do not give rise to the generation of "new" knowledge per se but rather synthesize or reiterate the ideas that are produced through other research activities.

In addition to these formal records of interaction, it is also important to consider more informal interactions such as day-to-day discussions that take place between individuals in a community of scientists and scholarly works that significantly influence a person's work. While data was collected measuring some facets of such informal interactions, evaluation and analyses of this data was outside the time frame and scope of this particular study. Nevertheless, a superficial assessment of these items did indicate some specific themes. In addition, to the overlap between the people with whom the participants' had already coauthored a number of new names and roles were identified. For example, scholars who currently work at institutions with sport management doctoral programs often cited their advisees as people with whom they frequently discuss research related topics. Also, scholars at a wide variety of institutions expressed a tendency to talk to other scholars at their institution about such things as research design, methodological issues, and statistical questions.

However, one of the most intriguing themes that surfaced was that scholars who mainly utilize positivistic, quantitative approaches often cited people and authors of specific scholarly works pertaining to their substantive areas and domains of interest as the people who have most influenced their work. Contrastingly, scholars who embrace more postpositivistic, qualitative approaches tended to nominate instructors and scholars who are known for their methodological advances (e.g., Patti Lather and Gloria LadsonBillings were identified by several participants). One might speculate that a reason for 
such results could be that those who adopt methodological approaches outside what might be considered "mainstream," are forced to really delve into the metaphysical and philosophical underpinnings behind their work (much like the Kuhnian ideas of periods of crisis and revolution). On the other hand, those who embrace more conventional methodological approaches are attempting to advance knowledge under the terms of a dominant paradigm (i.e., engaging in practices under the guise of a Kuhnian period of Normal Science).

\section{Future Research Directions}

Studying the social structural organization of scientific activities and the social construction of knowledge has garnered a lot of attention over the past 30-40 years in a variety of disciplines (e.g., Abbot, 2001; Denzin \& Lincoln, 2005). Findings from these studies indicate that there are numerous social factors that influence the development and structures of scientific practices and knowledge growth (e.g., Cappell \& Guterbock, 1992; Collins, 2000; Ennis, 1992). While sport management scholars have often discussed the value and implications of such social interactions as advisor and peer mentoring relationships (e.g., Pastore, 2003), publications in peer-reviewed journals (Chelladurai, 2005), and membership in academic professional organizations (i.e., NASSM) (Pitts, 2001) in an ideological sense, no empirical studies until now have explored how these social facets play out in terms of the structural constraints and opportunities such patterns of interaction hold for the idea space in the field.

That being said, in this study, we did not actually study the idea space in the field. While describing who affiliates with what groups and who works with whom offers a 
unique and robust understanding of the underlying social structure of a discipline, it provides very little information about how the social structure is actually tied to the idea space and topics circulating within the structure. Therefore many questions can be raised tying the social and cognitive structures of the field. What is the content of the works these scholars are actually producing? Who are they citing? What theories, frameworks and paradigms are they using as lenses for viewing the world? Are they truly building off of one another's work? Are Institution "Q" affiliates really more heterogeneous in the topics they study and the approaches they employ?

These are just a few of the many questions that come to mind in looking to further our understanding of the social construction of knowledge in the field of sport management. While it is difficult to produce a transparent mapping of the idea space and the actors producing the ideas in a field of study, we can use the formal coauthorship network obtained in this study as a foundation upon which to build our understanding of the social, political, ideological forces that are shaping the ideas and practices of sport management. 
APPENDIX A 
February 10, 2006

Dear Colleague:

We are conducting a study on the social construction of knowledge in the field of sport management. Specifically, we are interested in the collaboration patterns among scholars who have published in the field. As a scholar who publishes in the field of sport management, we are inviting you to participate in a research study examining this topic. Your input is critical in assisting with this important study as we are attempting to construct as complete a picture of the collaboration structure in the field of sport management as possible.

Studies in the sociology of science suggest that some of the most important indicators of social organization in research areas include informal discussions of pertinent research topics, relationships between advisors and doctoral students, and collaboration on publications. Likewise, studies have suggested that there is a direct linkage between patterns of social interaction, the structure of idea spaces, and the technical practices of a scientific discipline. Thus, fundamentally, the purpose of the study is to investigate the social interactions and underlying social structures of the scientific community of sport management scholars. The data collected will be used to construct co-authorship networks in the discipline and describe trends in the sport management collaboration network over time.

The derived network structures will be analyzed using a methodological approach called social network analysis and be compared to three competing theoretical models of scientific knowledge growth and disciplinary structures. As each of these models proposes unique theoretical and conceptual implications for the idea space and practices of a scientific discipline, the structure of collaboration patterns in sport management will ultimately be tied to claims about scientific practices posited by the closest-fitting model.

This study is being conducted as part of a doctoral dissertation research project by Catherine Quatman and Dr. Packianathan Chelladurai. Your participation in this study is voluntary. You may withdraw from the study at any point without penalty and may refuse to answer any questions that make you feel uncomfortable. By returning this survey, you consent to participate. Although participants receive no personal benefit or reward for their participation, your responses will contribute to the expanding sport management knowledge base.

It is anticipated your participation in this study will take approximately $\mathbf{5}$ minutes to complete and is composed of two parts. Part I of the study asks you to attach an upto-date copy of your curriculum vita. Part II of the study asks you to answer six openended questions about the social interactions and influences related to your research practices. 
Please be assured that your responses will be kept confidential. The data will be recorded in such a way to protect the confidentiality of the participants. Specifically, the actual actor names will be coded into a number for the analysis stages of the procedures. In addition, pseudonyms will be used to protect the subjects' confidentiality in all reporting procedures (i.e., results and discussions in all publications and presentations). As such, the items on the instrument will not place subjects at risk of criminal or civil liability or be damaging to the subjects' financial standing, employability, or reputation.

Your specific responses will not be linked to any individual or institution, and any discussion will be based only on group data. If you have any questions concerning your research rights or the study at any time, contact Catherine Quatman at the number listed below. Please complete and return the survey and attach your curriculum vita in an email response to quatman.8@osu.edu. If you would rather submit your responses through the postal mail service, please send your response to the address given below. Thank you for your cooperation.

Sincerely,

Catherine Quatman

Doctoral Candidate

quatman.8@osu.edu

(814) 440-2598

248 Cunz Hall-SFHP

1841 Millikin Road

The Ohio State University

Columbus, OH 43210

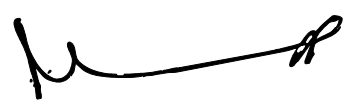

P. Chelladurai, Ph.D.

Professor

The Ohio State University 


\section{Part I.}

Please send an attached copy of your current curriculum vita. By supplying us with this document, you will be providing us with a large amount of data with little demand on your time. Below is a description of how we plan to use the information from your vita. Please remember, your privacy and confidentiality will be preserved as all of your information will be coded into a subject number and reported under a pseudonym.

As the main focus of this investigation is to construct a collaboration network based upon co-authorship on publications, curriculum vitae can serve as a direct means for obtaining information on the papers a particular researcher has published. In addition, curriculum vitae also provide in-depth description about scholars' educational and work backgrounds such as the years and institutions in which researchers earned their educational degrees. The information provided will be coded into formal variables to explore collaboration patterns in the field (e.g., institutional affiliation, number of peerreviewed publications, and time in the field).

To explore co-authorship patterns in the discipline, two types of networks will be constructed using graph theoretical principles of nodes (actors) and edges (ties between them). The first is a one-mode network used to represent the structure of co-authorship contacts in general. In this regard, the foundational relation of interest is essentially binary and pertains to whether or not two scholars in the network have ever co-authored together (regardless of how often they have co-authored together).

While the one-mode network corresponding to the unique co-authorship patterns provides interesting implications, it does not provide information on the relative strength or frequency of collaboration between authors. In order to examine this aspect of the collaboration patterns in the discipline of sport management, a two-mode network using actors as one set of nodes and publications as another set of nodes will be constructed. This network graph will capture a sense of how strongly collaborators are connected to each other in terms of how often they have published together. 


\section{Part II.}

Defining the structure of a discipline based solely on co-authorship patterns on formal publications is only one of many indicators of the interactive activities that constitute the potential for social influence in a scientific discipline. For example, a researcher's work can be influenced indirectly by the work and authors that they read and cite. Likewise, informal discussions can take place between actors in the network that are highly influential yet no publication or formal record of their relational interaction may ensue. Thus, the following items seek to delve further into the other types of social interaction and influence in the scientific activities taking place in the field of sport management. Once again, your privacy and confidentiality will be preserved as all of the information you provide us with will be coded into subject numbers and reported under a pseudonym.

A) Please list the names of the people with whom you believe you are most likely to collaborate on peer-reviewed publications. You can list as many (more than 5 if relevant) or as few (less than 5 if relevant) as you believe to be likely collaborators. You may also list individuals who may not necessarily be viewed as sport management scholars. After their name please give a general indication of why it is you are likely to work with them. You can list as many or as few reasons as you believe to be relevant to your decision to collaborate with them.

For example:

Mary Jones: my doctoral advisor, a great statistician, interested in the same topic John Smith: colleague at my current institution, friend, we work well together

1.

2.

3.

4.

5 . 
B) Please list the names of people with whom you most frequently discuss topics related to your research. You can list as many (more than 5 if relevant) or as few people (less than 5 if relevant) as you believe qualify as people you often interact with in this regard. Your list may include people from outside the field of sport management. People you listed in question 1 as well as any additional people can be included in your list. In addition, after their names please list the topics you most often discuss.

For example:

Mary Jones: social learning theory, intercollegiate athletics, diversity issues John Smith: consumer behavior, fan identification, qualitative methods

1.

2.

3.

4.

5.

C) Please list the top five people whom you believe to have greatly influenced your work. These people do not have to be someone who you have personally or directly interacted with nor someone in the field of sport management, but can also be for example someone you often cite or an instructor that directly impacted your research interests, etc. After their name, please list their relationship to your work.

For example:

Mary Jones: advisor, introduced me to social identity theory

John Smith: researcher I often cite, very influential in sport marketing research

1.

2.

3.

4.

5. 
D) Please provide the name of your doctoral advisor.

E) Please check the categories of research domains, topics, and methodological approaches where you believe your work best fits (check all that apply).

Intercollegiate Athletics

High School Sports

Youth Sports

Recreational Sport

Professional Sport

Community Sport

Olympic Sport

Adapted/Paralympic Sport/Activity

Consumer Behavior

Sport Marketing

Organizational Theory

Organizational Behavior

General Management

Ethics

Facility Operations

Event Management

Financial Aspects in Sport

Professional Preparation/Development

Methodology

Leadership

Motivation

Organizational Justice

Organizational Commitment

Sponsorship

Diversity Issues

Fan Identification

Spectator Motives

Sponsorship/Donor Behavior 
Sport Tourism
Survey
Case Studies
Experimental
Ethnography
Interviews
Focus Groups
Observation

_uantitative Methods

Qualitative Methods

Mixed Methods

_Other

Please specify:

\section{F) In your own words, please describe the primary focuses of your research throughout your career.}

For example:

My research has primarily focused on understanding community sport settings from a critical theory perspective. I have commonly employed qualitative methods and occasionally engaged in quantitative survey research.

My research has primarily focused on:

Thank you for your time and cooperation. If you have any questions about this project or would like to receive information about the results of this study, please contact Catherine Quatman at quatman.8@osu.edu or (814) 440-2598. 


\section{Participant Descriptive Statistics}

\section{Statistics}

\begin{tabular}{|rl|r|r|r|}
\hline & \multicolumn{1}{|c|}{$\begin{array}{c}\text { Years in } \\
\text { the Field }\end{array}$} & $\begin{array}{c}\text { Number of } \\
\text { Publications }\end{array}$ & $\begin{array}{c}\text { Unique } \\
\text { Coauthors }\end{array}$ \\
\hline $\mathrm{N} \quad$ Valid & 77 & 77 & 77 \\
& Missing & 0 & 0 & 0 \\
\hline
\end{tabular}

Years in the Field

\begin{tabular}{|c|c|c|c|c|c|}
\hline & & Frequency & Percent & Valid Percent & $\begin{array}{c}\text { Cumulative } \\
\text { Percent }\end{array}$ \\
\hline \multirow[t]{28}{*}{ Valid } & 1.00 & 4 & 5.2 & 5.2 & 5.2 \\
\hline & 2.00 & 4 & 5.2 & 5.2 & 10.4 \\
\hline & 3.00 & 1 & 1.3 & 1.3 & 11.7 \\
\hline & 4.00 & 3 & 3.9 & 3.9 & 15.6 \\
\hline & 5.00 & 7 & 9.1 & 9.1 & 24.7 \\
\hline & 6.00 & 7 & 9.1 & 9.1 & 33.8 \\
\hline & 7.00 & 6 & 7.8 & 7.8 & 41.6 \\
\hline & 8.00 & 4 & 5.2 & 5.2 & 46.8 \\
\hline & 9.00 & 5 & 6.5 & 6.5 & 53.2 \\
\hline & 10.00 & 1 & 1.3 & 1.3 & 54.5 \\
\hline & 11.00 & 3 & 3.9 & 3.9 & 58.4 \\
\hline & 12.00 & 1 & 1.3 & 1.3 & 59.7 \\
\hline & 15.00 & 4 & 5.2 & 5.2 & 64.9 \\
\hline & 16.00 & 4 & 5.2 & 5.2 & 70.1 \\
\hline & 18.00 & 2 & 2.6 & 2.6 & 72.7 \\
\hline & 19.00 & 4 & 5.2 & 5.2 & 77.9 \\
\hline & 20.00 & 1 & 1.3 & 1.3 & 79.2 \\
\hline & 21.00 & 4 & 5.2 & 5.2 & 84.4 \\
\hline & 22.00 & 1 & 1.3 & 1.3 & 85.7 \\
\hline & 24.00 & 1 & 1.3 & 1.3 & 87.0 \\
\hline & 25.00 & 1 & 1.3 & 1.3 & 88.3 \\
\hline & 26.00 & 3 & 3.9 & 3.9 & 92.2 \\
\hline & 27.00 & 1 & 1.3 & 1.3 & 93.5 \\
\hline & 29.00 & 1 & 1.3 & 1.3 & 94.8 \\
\hline & 30.00 & 1 & 1.3 & 1.3 & 96.1 \\
\hline & 31.00 & 1 & 1.3 & 1.3 & 97.4 \\
\hline & 33.00 & 2 & 2.6 & 2.6 & 100.0 \\
\hline & Total & 77 & 100.0 & 100.0 & \\
\hline
\end{tabular}




\section{Number of Publications}

\begin{tabular}{|c|c|c|c|c|c|}
\hline & & Frequency & Percent & Valid Percent & $\begin{array}{c}\text { Cumulative } \\
\text { Percent }\end{array}$ \\
\hline \multirow[t]{40}{*}{ Valid } & 1.00 & 3 & 3.9 & 3.9 & 3.9 \\
\hline & 2.00 & 2 & 2.6 & 2.6 & 6.5 \\
\hline & 3.00 & 4 & 5.2 & 5.2 & 11.7 \\
\hline & 4.00 & 4 & 5.2 & 5.2 & 16.9 \\
\hline & 5.00 & 4 & 5.2 & 5.2 & 22.1 \\
\hline & 6.00 & 6 & 7.8 & 7.8 & 29.9 \\
\hline & 7.00 & 2 & 2.6 & 2.6 & 32.5 \\
\hline & 8.00 & 2 & 2.6 & 2.6 & 35.1 \\
\hline & 9.00 & 1 & 1.3 & 1.3 & 36.4 \\
\hline & 10.00 & 5 & 6.5 & 6.5 & 42.9 \\
\hline & 12.00 & 1 & 1.3 & 1.3 & 44.2 \\
\hline & 13.00 & 4 & 5.2 & 5.2 & 49.4 \\
\hline & 14.00 & 1 & 1.3 & 1.3 & 50.6 \\
\hline & 16.00 & 1 & 1.3 & 1.3 & 51.9 \\
\hline & 17.00 & 2 & 2.6 & 2.6 & 54.5 \\
\hline & 18.00 & 1 & 1.3 & 1.3 & 55.8 \\
\hline & 19.00 & 1 & 1.3 & 1.3 & 57.1 \\
\hline & 20.00 & 4 & 5.2 & 5.2 & 62.3 \\
\hline & 21.00 & 1 & 1.3 & 1.3 & 63.6 \\
\hline & 22.00 & 3 & 3.9 & 3.9 & 67.5 \\
\hline & 24.00 & 1 & 1.3 & 1.3 & 68.8 \\
\hline & 26.00 & 2 & 2.6 & 2.6 & 71.4 \\
\hline & 27.00 & 3 & 3.9 & 3.9 & 75.3 \\
\hline & 29.00 & 2 & 2.6 & 2.6 & 77.9 \\
\hline & 30.00 & 1 & 1.3 & 1.3 & 79.2 \\
\hline & 32.00 & 2 & 2.6 & 2.6 & 81.8 \\
\hline & 38.00 & 1 & 1.3 & 1.3 & 83.1 \\
\hline & 39.00 & 1 & 1.3 & 1.3 & 84.4 \\
\hline & 42.00 & 1 & 1.3 & 1.3 & 85.7 \\
\hline & 43.00 & 2 & 2.6 & 2.6 & 88.3 \\
\hline & 47.00 & 1 & 1.3 & 1.3 & 89.6 \\
\hline & 52.00 & 1 & 1.3 & 1.3 & 90.9 \\
\hline & 56.00 & 1 & 1.3 & 1.3 & 92.2 \\
\hline & 67.00 & 1 & 1.3 & 1.3 & 93.5 \\
\hline & 69.00 & 1 & 1.3 & 1.3 & 94.8 \\
\hline & 71.00 & 1 & 1.3 & 1.3 & 96.1 \\
\hline & 76.00 & 1 & 1.3 & 1.3 & 97.4 \\
\hline & 79.00 & 1 & 1.3 & 1.3 & 98.7 \\
\hline & 93.00 & 1 & 1.3 & 1.3 & 100.0 \\
\hline & Total & 77 & 100.0 & 100.0 & \\
\hline
\end{tabular}


Unique Coauthors

\begin{tabular}{|c|c|c|c|c|c|}
\hline & & Frequency & Percent & Valid Percent & $\begin{array}{c}\text { Cumulative } \\
\text { Percent }\end{array}$ \\
\hline \multirow[t]{35}{*}{ Valid } & 1.00 & 4 & 5.2 & 5.2 & 5.2 \\
\hline & 2.00 & 6 & 7.8 & 7.8 & 13.0 \\
\hline & 3.00 & 4 & 5.2 & 5.2 & 18.2 \\
\hline & 4.00 & 3 & 3.9 & 3.9 & 22.1 \\
\hline & 5.00 & 7 & 9.1 & 9.1 & 31.2 \\
\hline & 6.00 & 4 & 5.2 & 5.2 & 36.4 \\
\hline & 7.00 & 3 & 3.9 & 3.9 & 40.3 \\
\hline & 8.00 & 1 & 1.3 & 1.3 & 41.6 \\
\hline & 9.00 & 3 & 3.9 & 3.9 & 45.5 \\
\hline & 10.00 & 3 & 3.9 & 3.9 & 49.4 \\
\hline & 12.00 & 2 & 2.6 & 2.6 & 51.9 \\
\hline & 13.00 & 5 & 6.5 & 6.5 & 58.4 \\
\hline & 15.00 & 1 & 1.3 & 1.3 & 59.7 \\
\hline & 17.00 & 2 & 2.6 & 2.6 & 62.3 \\
\hline & 18.00 & 3 & 3.9 & 3.9 & 66.2 \\
\hline & 19.00 & 3 & 3.9 & 3.9 & 70.1 \\
\hline & 20.00 & 3 & 3.9 & 3.9 & 74.0 \\
\hline & 21.00 & 2 & 2.6 & 2.6 & 76.6 \\
\hline & 22.00 & 1 & 1.3 & 1.3 & 77.9 \\
\hline & 23.00 & 1 & 1.3 & 1.3 & 79.2 \\
\hline & 24.00 & 1 & 1.3 & 1.3 & 80.5 \\
\hline & 25.00 & 2 & 2.6 & 2.6 & 83.1 \\
\hline & 27.00 & 1 & 1.3 & 1.3 & 84.4 \\
\hline & 28.00 & 2 & 2.6 & 2.6 & 87.0 \\
\hline & 29.00 & 1 & 1.3 & 1.3 & 88.3 \\
\hline & 30.00 & 1 & 1.3 & 1.3 & 89.6 \\
\hline & 33.00 & 1 & 1.3 & 1.3 & 90.9 \\
\hline & 35.00 & 1 & 1.3 & 1.3 & 92.2 \\
\hline & 38.00 & 1 & 1.3 & 1.3 & 93.5 \\
\hline & 41.00 & 1 & 1.3 & 1.3 & 94.8 \\
\hline & 53.00 & 1 & 1.3 & 1.3 & 96.1 \\
\hline & 54.00 & 1 & 1.3 & 1.3 & 97.4 \\
\hline & 56.00 & 1 & 1.3 & 1.3 & 98.7 \\
\hline & 83.00 & 1 & 1.3 & 1.3 & 100.0 \\
\hline & Total & 77 & 100.0 & 100.0 & \\
\hline
\end{tabular}




\section{Years in the Field}

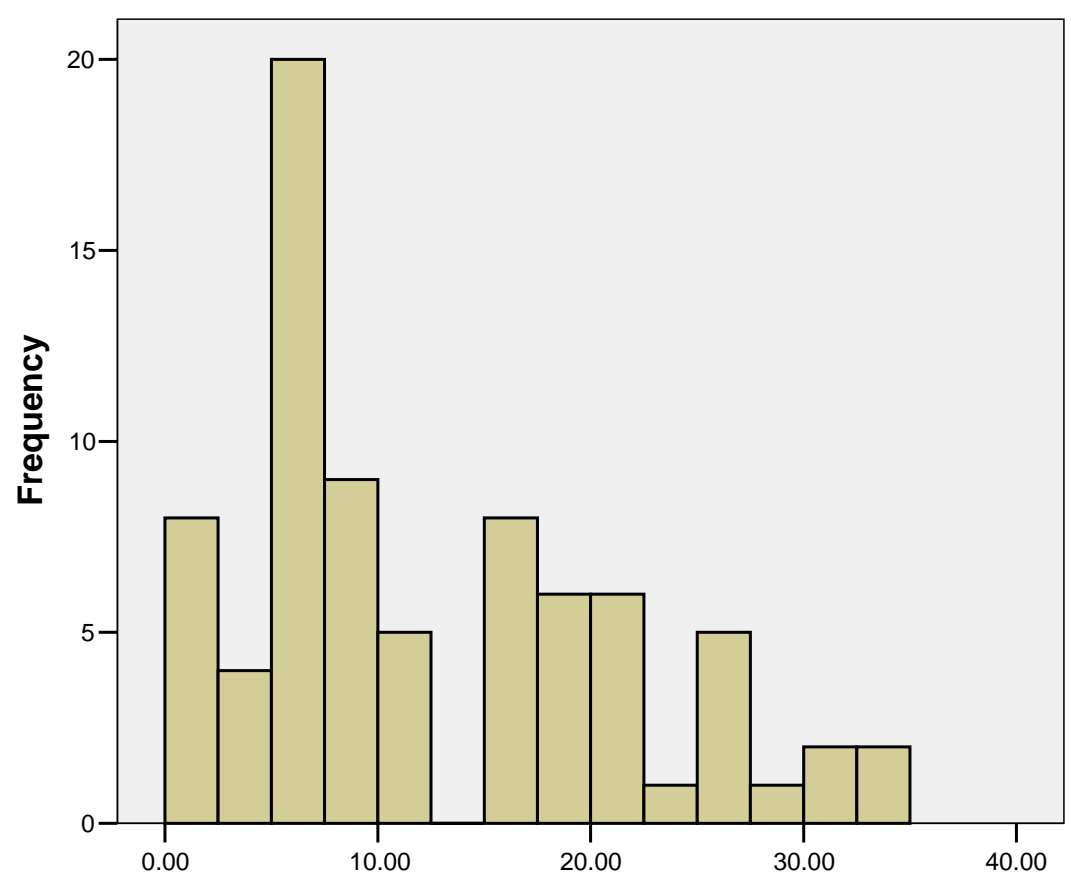

Mean $=12.3377$

Std. Dev. $=8.77138$

$\mathrm{N}=77$

\section{Number of Publications}

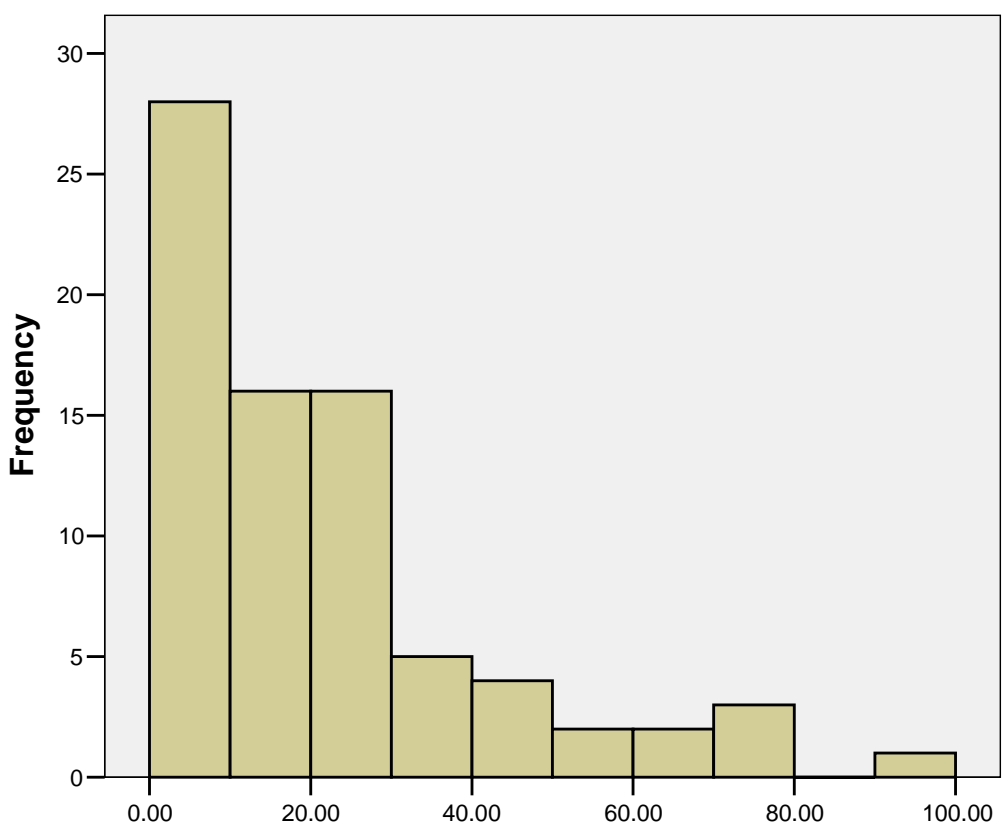

Mean $=21.2468$

Std. Dev. $=20.73654$

$\mathrm{N}=77$ 


\section{Unique Coauthors}

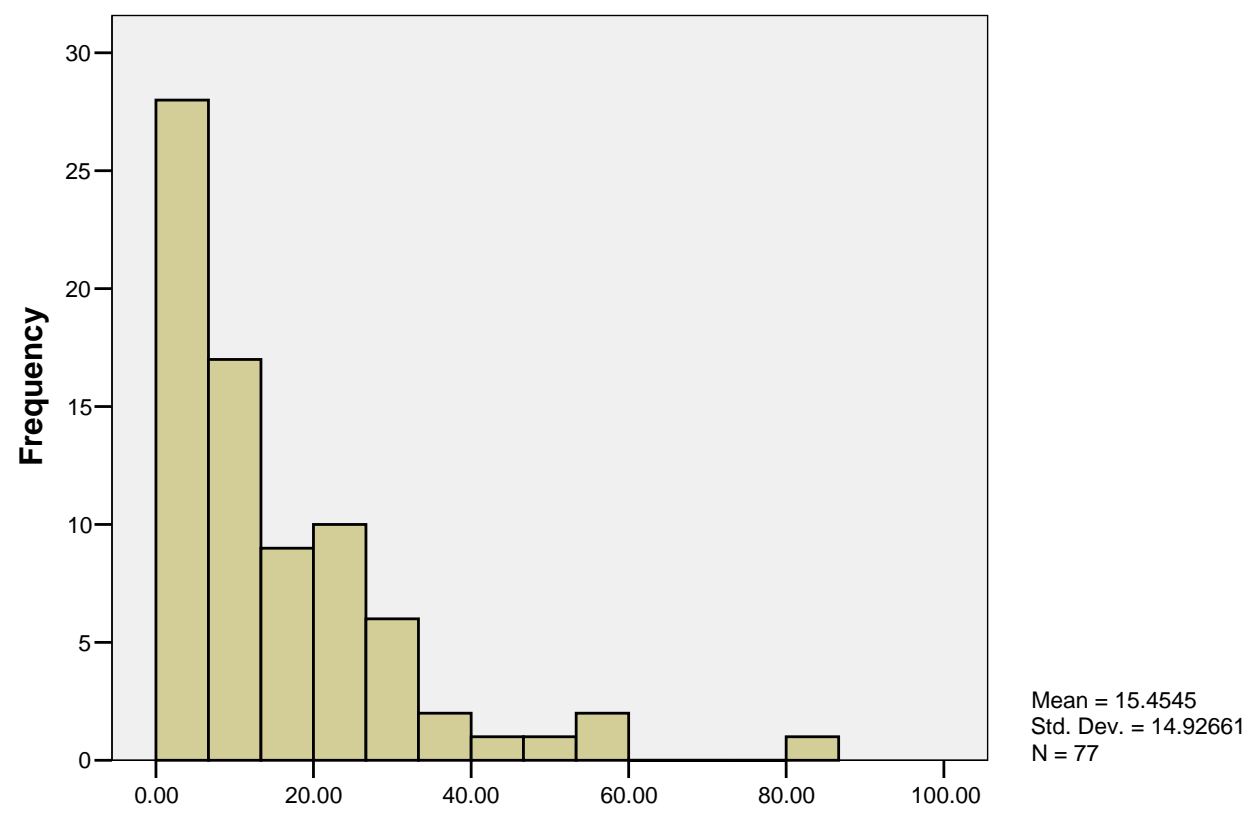




\section{REFERENCES}

Abbott, A. (2000). Reflections on the future of sociology. Contemporary Sociology, 29, 296-300.

Abbott, A. (2001). Chaos of Disciplines. Chicago, IL: University of Chicago Press.

Alexander, J.C. (1987). Twenty Lectures: Sociological Theory Since World War II. New York: Columbia University Press.

Amis, J. \& Silk, M. (2005). Rupture: Promoting critical and innovative approaches to the study of sport management. Journal of Sport Management, 19, 355-366.

Barabasi, A. \& Albert, R. (1999). Emergence of scaling in random networks. Science, 286, 509-512.

Batagelj, V. \& Mrvar, A. (1996). Pajek 1.02: Software for Network Analysis and Visualization. Available free for non-commercial use at http://vlado.fmf.unilj.si/pub/networks/pajek/default.htm and through Ucinet 6 software program.

Berkowitz, S.D. (1982). Introduction to Structural Analysis: The Network Approach to Social Research. Toronto, Canada: Butterworth \& Co. Ltd.

Borgatti, S.P., Everett, M.G. \& Freeman, L.C. (2002). Ucinet for Windows: Software for Social Network Analysis. Harvard, MA: Analytic Technologies.

Boucher, R.L. (1998). Toward achieving a focal point for sport management: A binocular perspective. Journal of Sport Management, 12, 76-85.

Brieger, R. (2004). The analysis of social networks. In M. Hardy and A. Bryman’s (Eds.) 
Handbook of Data Analysis. London: Sage Publications, Pp. 505-526.

Breiger, R. (1974). The duality of persons and groups. Social Forces, 53, p. 181-190.

Costa, C.A. (2005). The status and future of sport management: A Delphi study. Journal of Sport Management, 19, 117-142.

Calhoun, C., Gerteis, J., Moody, J., Praff, S. Schmidt, K., \& Virk, I. (2002). Classical Sociological Theory. Malden, MA: Blackwell Publishing Ltd.

Cappell, C.L. \& Guterbock, T.M. (1992). Visible colleges: The social and conceptual structure of sociology specialties. American Sociological Review, 57, 266-273.

Chalip, L. (2006). Toward a distinctive sport management discipline. Journal of Sport Mangement, 20, 1-21.

Chelladurai, P. (2005). Managing Organizations for Sport and Physical Activity: A Systems Perspective ( $2^{\text {nd }}$ ed.). Scottsdale, AZ: Holcomb Hathaway, Publishers, Inc.

Collins, H.M. (1998). The meaning of data: open and closed evidential cultures in the search for gravitational waves. American Journal of Sociology, 104, 293-338.

Crane, D. (1988). Invisible Colleges: Diffusion of Knowledge in Scientific Communities. Chicago, IL: The University of Chicago Press.

Cuneen, J. (2004). Managing program excellence during our transition from potential to merit. Journal of Sport Management, 18, 1-12.

Daipha, P. (2001). The intellectual and social organization of ASA 1990-1997:

Exploring the interface between the discipline of sociology and its practitioners. The American Sociologist, 32, 73-90.

Daprano, C.M., Bruening, J.E., Pastore, D.L., Greenwell, C., Dixon, M.A., Ko, J.K., 
Jordan, J.S., Lilienthal, S.K., Turner, B.A. (2005). Collaboration in sport research: A case from the field. Quest, 57, 300-314.

De Nooy, W., Mrvar, A. \& Batagelj, V. (2005). Exploratory Social Network Analysis with Pajek. New York, NY: Cambridge University Press.

Denzin, N. K. \& Lincoln, Y.S. (Eds.). (2003). The Landscape of Qualitative Research: Theories and Issues. Second Edition. Thousand Oaks, CA: Sage Publications, Inc.

Dickson, D. (1997). Champions or challengers of the cause of science? Nature, 387, 333324.

Ede, L. \& Lunsford, A. (1990). Single Texts/Plural Authors: Perspectives on Collaborative Writing. Carbondale: Southern Illinois University Press.

Emirbayer, M. (1997). Manifesto for a relational sociology. The American Journal of Sociology, 103(2), 281-317.

Endersby, J.W. (1996). Collaborative research in the social sciences: Multiple authorship and publication credit. Social Science Quarterly, 77(2), 375-392.

Ennis, J.G. (1992). The social organization of sociological knowledge: Modeling the intersection of specialties. American Sociological Review, 57, 259-265.

Fraenkel, J.R. \& Wallen, N.E. (2003). How to Design and Evaluate Research in Education. New York, NY: McGraw-Hill Companies, Inc.

Freeman, L. (2004). The Development of Social Network Analysis: A Study in the Sociology of Science. Vancouver, Canada: Empirical Press.

Frisby, W. (2005). The good, the bad, and the ugly: Critical sport management research. Journal of Sport Management, 19, 1-12. 
Friedkin, N.E. (1998). A Structural Theory of Social Influence. Cambridge, UK: Cambridge University Press.

Fuchs, S. (1993). A sociological theory of scientific change. Social Forces, 71(4), 933953.

Fuchs, S. \& Ward, S. (1994). What is deconstruction, and where and when does it take place? Making facts in science, building cases in law. American Sociological Review, 481-500.

Glesne, C. (2006). Becoming Qualitative Researchers: An Introduction. Boston, MA: Pearson Education, Inc.

Granovetter, M. (1992). Problems of explanation in economic sociology. In N. Nohria \& R.G. Eccles' (Eds.) Networks and Organizations: Structure, Form and Action. Boston, MA: Harvard Business School Press. Pp. 25-56.

Green, J. \& Stinson, S. (1999). Postpositivist Research in Dance. In S.H. Fraleigh and P. Hanstein's (Eds.) Researching Dance: Evolving Modes of Inquiry. University of Pittsburgh Press, Pp. 91-123.

Hanneman, R.A. \& Riddle, M. (2005). Introduction to Social Network Methods. Riverside, CA: University of California, Riverside. (published in digital form at http://faculty.ucr.edu/ hanneman/).

Hargens, L.L. (1988). Scholarly consensus and journal rejection rates. American Sociological Review, 53, 139-151.

Hollis, M. (1994). The Philosophy of Social Science: An Introduction. New York, NY: Cambridge University Press.

Homans, G.C. (1962). The Nature of Social Science. New York, NY: An Original Harbinger Book, Harcourt, Brace \& World, Inc. 
James, J.D. (2001). The role of cognitive development and socialization in the initial development of team loyalty. Leisure Sciences, 23, 233-261.

Kilduff, M., \& Tsai, W. (2005). Social Networks and Organizations. London, UK: Sage Publications.

Kuhn, T.S. (1962; 1970). The Structure of Scientific Revolutions. Chicago, IL: The University of Chicago Press.

Lather, P. (1986). Research as Praxis. Harvard Educational Review, 56(3), 257-277.

Laumann, E.O., Marsden, P.V., \& Prensky, D. (1983). The boundary specification problem of network analysis. In R.S. Burt \& M. J. Minor’s (Eds.) Applied Network Analysis: A Methodological Introduction, Beverly Hills, CA: Sage Publications, Pp. 18-34.

Laverie, D. A. \& Arnett, D.B. (2000). Factors affecting fan attendance: The influence of salience and satisfaction. Journal of Leisure Research, 32(2), 225-246.

Martin, J. (2002). Power, authority, and the constraints of belief systems. American Journal of Sociology, 107, 861-904.

Merton, R.K. (1968). The Matthew effect in science. Science, 159, 56-63.

Mizruchi, M.S. \& Fein, L.C. (1999). The social construction of organizational knowledge: A study of the uses of coercive, mimetic, and normative isomorphism. Administrative Science Quarterly, 44, 653-683.

Moody, J. (2004). The structure of a social science collaboration network: Disciplinary cohesion from 1963 to 1999. American Sociological Review, 69, 213-238.

Moody, J. \& Leahey, E. (2006). Network foundations for the diffusions of innovations.

Moody, J. \& White, D.R. (2003). Social Cohesion and embeddedness: A hierarchical 
conception of social groups. American Sociological Review, 68, 103-127.

Moody (2000). The importance of relationship timing for diffusion. Social Forces, 81, 25-56.

Mullins, N.C. (1973). Theories and Theory Groups in Contemporary Sociology. New York: Harper and Row.

Nohria, N. (1992). Introduction. In N. Nohria \& R.G. Eccles’ (Eds.) Networks and Organizations: Structure, Form, and Action. Boston, MA: Harvard Business School Press. Pp. 1-22.

Olafson, G.A. (1995). Sport management research: Ordered change. Journal of Sport Management, 9(3), 338-345.

Owen-Smith, J. (2001). Managing laboratory work through skepticism Processes of evaluation and control. American Sociological Review, 66, 427-452.

Pastore, D.L. (2003). A different lens to view mentoring in sport management. Journal of Sport Management, 17, 1-12.

Persoon, O., Glanzel, W., \& Danell, R. (2004). Inflationary bibliometric values: The role of scientific collaboration and the need for relative indicators in evaluation studies. Scientometrics, 60(3), 421-432.

Pitts, B.G. (2001). Sport management at the millennium: A defining moment. Journal of Sport Management, 15, 1-9.

Price, D.J. (1986). Little Science, Big Science ... and Beyond. New York: Columbia University Press.

Rogers, E. M. (2003). Diffusion of Innovations. New York, NY: Free Press.

Schwandt, T.A. (2001). Dictionary of Qualitative Inquiry. Thousand Oaks, CA: Sage 
Publications, Inc.

Scott, J. (2001). Social Network Analysis: A Handbook. Thousand Oaks, CA: Sage Publications, Inc.

Skinner, J. \& Edwards, A. (2005). Inventive pathways: Fresh visions of sport management research. Journal of Sport Management, 19, 404-421.

Slack, T.S. (1998). Is there anything unique about sport management? European Journal for Sport Management, 5(2), 21-29.

Slack, T. (1996). From the locker room to the board room: Changing the domain of sport management. Journal of Sport Management, 10, 97-105.

Small, H., \& Crane, D.G. (1979). Specialties and disciplines in science and social science: An examination of the their structure using citation indexes, Scientometrics, 1, 445-461.

Solomon, R.C. (2002). The Big Questions: A Short Introduction to Philosophy. Canada: Wadsworth Group/Thomson Learning.

Swidler, A. \& Arditi, J. (1994). The new sociology of knowledge. Annual Review of Sociology, 20, 305-329.

Usher, R. (1996). A critique of the neglected epistemological assumptions of educational research. In D. Scott and R. Usher's (Eds.) Understanding Educational Reasearch. New York, NY: Routledge.

Wasserman, S. \& Faust, K. (1994). Social Network Analysis: Methods and Applications. Cambridge, UK: Cambridge University Press.

Weese, W.J. (2002). Opportunities and headaches; Dichotomous perspectives on the current and future hiring realities in the sport management academy. Journal of Sport Management, 16, 1-17. 
Wellman, B. (1988). Structural analysis: From metaphor to substance. In B. Wellman and S.D. Berkowitz’s (Eds.) Social Structures: A Network Approach. New York, NY: Cambridge University Press.

Wellman, B. \& Berkowitz, S.D. (1988). Studying social structures. In B. Wellman and S.D. Berkowitz's (Eds.) Social Structures: A Network Approach. New York, NY: Cambridge University Press.

White, D.R., Owen-Smith, J., Moody, J. \& Powell, W.W. (2004). Networks, Fields and Organizations. Computational and Mathematical Organization Theory, 10, 95117. 